UNIVERSIDADE DE SÂO PAULO

INSTITUTO DE FÍSICA

\title{
Modelos Seesaw a Baixas Energias e Modelo de Violação Mínima de Sabor no Modelo Seesaw Tipo III
}

\author{
Lindber Ivan Salas Escobar
}

Dissertação apresentada ao Instituto de Física da Universidade de São Paulo para a obtenção do titulo de Mestre em Ciências

Orientador: Prof. Dr. Oscar José Pinto Éboli

Comissão Examinadora:

Prof. Dr. Gustavo Alberto Burdman (IFUSP)

Prof ${ }^{\mathrm{a}}$. Dra ${ }^{\mathrm{a}}$. Marina Nielsen (IFUSP)

Prof. Dr. José Kenichi Mizukoshi (UFABC)

São Paulo

2012 


\section{FICHA CATALOGRÁFICA}

Preparada pelo Serviço de Biblioteca e Informação do Instituto de Física da Universidade de São Paulo

Escobar, Lindber Ivan Salas

Modelos seesaw a baixas energias e modelo de violação mínima de sabor no modelo seesaw tipo III. - São Paulo, 2012.

Dissertação (Mestrado) - Universidade de São Paulo. Instituto de Física, Depto. de Física Matemática

Orientador: Prof. Dr. Oscar José Pinto Éboli Área de Concentração: Física

Unitermos: 1. Física de Partículas; 2. Física Teórica, 3. Física de Alta Energia.

USP/IF/SBI-083/2012 


\section{Agradecimentos}

Gostaria de agradecer primeiramente a meu tio Wilber pelo constante apoio e incentivo para alcançar este alvo, à minha família, à minha mãe Armida, à minha irmã Adriana a todos meus primos que são a motivação para seguir adiante. Gostaria de também agradecer:

- Ao meu orientador o Prof. Oscar Éboli, por sempre acreditar em mim e brindar a oportunidade de fazer este trabalho.

- À USP por me prover do ambiente adequado para o desenvolvimento da minha pesquisa.

- À CAPES pelo suporte financeiro.

- À Maristela Poter, Andre e Luana pela ajuda da revisão ortográfica desta dissertação.

- Aos meus colegas e amigos, em especial ao chato Jose e Denis colorado eles foram muitos importantes, pela ajuda e motivação para viajar a Brasil, ao Leonardo pelas conversas sobre campos e partículas ao Miguel, Fernando, Julio o judio, entre outros. Eles foram muitos importantes pela ajuda e companhia durante estes dois largos anos.

- Aos meus professores no IFUSP e ao meu professor Pereyra pela motivação de deixar meu pais Peru para continuar com meus estudos em este mundo apaixonante que é a Física de Partículas. 


\section{Resumo}

Enquanto todos os modelos com neutrinos massivos de Majorana levam ao mesmo operador efetivo de dimensão $d=5$, que não conserva número leptônico, os operadores de dimensão $d=6$, obtidos a baixas energias, conservam número leptônico e são diferentes dependendo do modelo de alta energia da nova física. Derivamos os operadores de dimensão $d=6$ que são característicos de modelos Seesaw genéricos, no qual a massa do neutrino resulta do intercâmbio de campos pesados que podem ser tanto singletos fermiônicos, tripletos fermiônicos ou tripletos escalares. Os operadores resultantes podem conduzir a efeitos observáveis no futuro próximo, se os coeficientes dos operadores de dimensão $d=5$ e $d=6$ são desacoplados. Neste trabalho apresentamos o modelo violação mínima de sabor no contexto do modelo seesaw tipo III, no qual é possível obter tal desacoplamento. Isto permite reconstruir a estrutura de sabor a partir dos valores das massas dos neutrino leves e dos parâmetros de mistura, mesmo na presença de fases de violação $\mathrm{CP}$. 


\section{Abstract}

While all models of Majorana neutrino masses lead to the same dimension five effective operator, which does not conserve lepton number, the dimension six operators induced at low energies conserve lepton number and differ depending on the high energy model of new physics. We derive the low-energy dimension six operators which are characteristic of generic Seesaw models, in which neutrino masses result from the exchange of heavy fields which may be either fermionic singlets, fermionic triplets or scalar triplets. The resulting operators may lead to effects observable in the near future, if the coefficients of the dimension five and six operators are decoupled. In this work we present the model of minimal flavor violation in the context of the type III seesaw model, in which it is possible to obtain the decoupling mentioned before. This allows to reconstruct the flavour structure of the model from the values of the light neutrino masses and mixing parameters, even in the presence of $\mathrm{CP}$-violating phases. 


\section{Sumário}

1 Introdução 1

2 Modelo Padrão 5

2.1 Modelo das interações eletrofracas . . . . . . . . . . . . . 5

2.1 .1 O Mecanismo de Higgs . . . . . . . . . . . . . . 8

2.1.2 Massa dos férmions e mistura . . . . . . . . . . . 14

2.2 Necessidade de Estender o Modelo Padrão: Não Conservação do Número leptônico e Mistura de Neutrinos . . . . . . . . . . 18

2.2 .1 Neutrino de Dirac e Majorana . . . . . . . . . . . . . 21

2.2 .2 Massa do Neutrino Tipo Dirac . . . . . . . . . . . 23

2.2.3 Massa do Neutrino Tipo Majorana . . . . . . . . . . . 24

3 Modelos Seesaw $\quad 27$

3.1 Seesaw Tipo I $\ldots \ldots \ldots \ldots \ldots \ldots \ldots$

3.1 .1 Operador de dimensão $d=5 \ldots \ldots \ldots$

3.1.2 Operador de dimensão $d=6 \ldots \ldots$. . . . . . . . 31

3.2 Seesaw Tipo II . . . . . . . . . . . . . . . . . . . . . 33

3.2.1 Operadores de dimensão $d=4$ e $d=5 \ldots \ldots . . .35$

3.2 .2 Operador de dimensão $d=6 \ldots \ldots$. . . . . . . 36

3.2 .3 Esquema de Renormalização . . . . . . . . . . . . . . 37

3.3 Seesaw Tipo III . . . . . . . . . . . . . . . . . . . 38

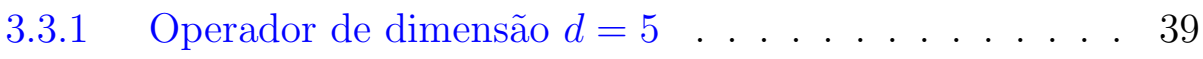

3.3.2 Operador de dimensão $d=6 \ldots \ldots$. . . . . . 40 
4 Fenomenologia $\quad 45$

4.1 Seesaw Tipo II . . . . . . . . . . . . . . . . . 45

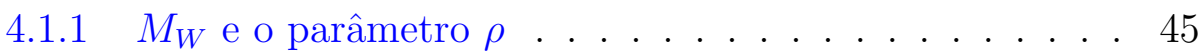

4.1.2 Decaimento de $\mu \rightarrow 3 e$ e $\tau \rightarrow 3 l \ldots . . . . . .47$

$4.1 .3 \quad l_{1} \rightarrow l_{2} \gamma \ldots \ldots \ldots \ldots$

4.2 Seesaw Tipo III . . . . . . . . . . . . . . . . . . . . . . . 50

4.2.1 Decaimento do $W$. . . . . . . . . . . 50

4.2 .2 Decaimento Invisível do $Z$. . . . . . . . . . . . . . . 51

4.2.3 Testes baseados em universalidade. . . . . . . . 52

4.2.4 Decaimento do $Z$ em léptons carregados . . . . . . . . 54

4.2.5 Decaimento de $\mu \rightarrow 3 e$ e $\tau \rightarrow 3 l \ldots . . . . . .55$

4.2.6 Lagrangiana completa do seesaw tipo III e $l_{1} \rightarrow l_{2} \gamma$. . 58

4.2.7 Combinação de todos os vínculos . . . . . . . . . . . . 60

4.3 Seesaw Tipo I . . . . . . . . . . . . . . . . . . 60

5 Violação Mínima de Sabor no Modelo Seesaw Tipo III 63

5.1 Introdução . . . . . . . . . . . . . . . . 63

5.2 MFV Seesaw tipo III . . . . . . . . . . . . . . . 65

5.2.1 Hierarquia entre as escalas $\Lambda_{F L}$ e $\Lambda_{L N}$. . . . . . . . . 66

5.2.2 Reconstrução dos acoplamentos de Yukawa $Y$ e $\tilde{Y}^{\prime}$. . . 66

5.2 .3 Hierarquia Normal . . . . . . . . . . . . . . . . 68

5.2 .4 Hierarquia Inversa . . . . . . . . . . . . . . . . . . . . 69

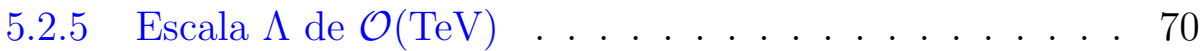

5.2.6 Autoestados de Massa e suas Interações . . . . . . . . 71

5.3 Fenomenologia .................. 74

$\begin{array}{lll}6 & \text { Conclusões } & 79\end{array}$

$\begin{array}{lr}\text { Apêndice } & 81\end{array}$ 


\section{Capítulo 1}

\section{Introdução}

O Modelo Padrão (MP) das interações eletrofracas proposto por Glashow, Salam e Weinberg [1]-[3] em meados dos anos 60 descreve com grande sucesso as interações eletromagnética e fraca em uma só teoria de gauge com uma simetria $S U(2)_{L} \times U(1)_{Y}$. Testes experimentais (a descoberta da interação fraca neutra [4], a produção dos bósons $W^{ \pm}$[5] e $Z$ [6], entre outros) nos últimos 40 anos têm confirmado as predições e precisão do MP, consolidando-o como a representação mais sólida das interações eletromagnética e fraca das partículas elementares.

Recentemente, experimentos de neutrinos revelaram que os neutrinos posuem massas não nulas [7], o que constitui evidência para uma física além do MP a uma escala de nova física ainda desconhecida (NF). O CERN Large Hadron Collider (LHC) começou a operação com o objetivo de explorar a física além do MP na escala TeV. Uma interessante questão é se a nova física associada às massas de neutrinos pode situar-se dentro do alcance do LHC e se esta nova física pode ser testada através de efeitos a baixas energias além de oscilação de neutrinos, como busca direta para as novas partículas envolvidas, larguras de decaimentos ou medidas electrofracas de precisão.

Efeitos de NF para baixas energias em relação a escala de NF podem ser descritos em termos de operadores efetivos de dimensão $d>4$ construídos com os campos do MP,

$$
\mathcal{L}_{\text {eff }}=\mathcal{L}_{M P}+c^{d=5} \mathcal{O}^{d=5}+c^{d=6} \mathcal{O}^{d=6}+\ldots
$$

Neste contexto apenas um operador de dimensão $d=5$ pode ser cons- 
truído [8],

$$
\mathcal{O}^{d=5}=\left(\overline{L_{\alpha L}^{c}} \widetilde{\phi}^{*}\right)\left(\widetilde{\phi}^{\dagger} L_{\beta L}\right),
$$

onde $L_{\alpha L}$ é o dubleto leptônico de $S U(2)_{L}$ e $\phi$ o dubleto de Higgs do MP. Este operador quebra a simetria de número leptônico total (NL), que é uma simetria acidental no MP. Depois da quebra espontânea da simetria, $\langle\phi\rangle=$ $v / \sqrt{2}=174 \mathrm{GeV}$, este termo dá origem a um termo de massa de Majorana para os neutrinos. Este operador é característico a todas as teorias com neutrinos de Majorana massivos. Portanto o coeficiente $c^{d=5}$ é fixado pela massa do neutrino.

Diferentes classes de modelos resultam em diferentes classes de operadores $\mathcal{O}^{d=6}$, assim sua identificação e eventualmente sua seleção experimental é muito importante para discriminar a origem da massa dos neutrinos. Uma importante propriedade destes operadores é que seus coeficientes $c^{d=6}$ podem não ser tão suprimidos como o coeficiente $c^{d=5}$, com o qual é possível observar efeitos a baixas energias. O fato de que todos os operadores de dimensão $d=6$ conservam NL em contraste com o único operador de dimensão $d=5$, indica que se pode obter de forma natural grandes coeficientes de $d=6$ resultantes de $\mathrm{NF}$, enquanto que os coeficientes de operadores que violam NL são pequenos. Tal possibilidade deveria indicar o desacoplamento dos coeficientes de dimensão $d=6$ com os de $d=5$ responsáveis pela massa dos neutrinos. Neste trabalho apresentamos o modelo com a violação mínima de sabor no contexto do modelo seesaw tipo III, no qual é possível obter tal desacoplamento. Esta monografia está organizada da seguinte forma.

No segundo capítulo deste trabalho é tratado o MP das interações eletrofracas, dando-se ênfase ao estudo das interações carregada e neutra para os léptons e introduzindo o mecanismo de Higgs para gerar massa aos férmions, assim como a mistura que acontece no setor dos quarks. Neste capítulo também apresentamos as diferenças entre neutrinos de Majorana e de Dirac no contexto de simetrias discretas, assim como o termo de massa de Dirac e Majorana para os neutrinos, e introduz-se a definição da matriz de Pontecorvo Maki Nakagawa Sakata, $U_{P M N S}$.

No terceiro capítulo apresentamos os modelos seesaw no contexto de uma teoria efetiva. No modelo seesaw tipo I são introduzidos novos estados neutros de mão direita $\left(N_{R}\right)$, modelo seesaw tipo II são adicionados tripletos escalares $(\Delta)$ de hipercarga $Y=1$ enquanto no modelo seesaw tipo III são adicionados tripletos de férmions com hipercarga $(Y=0)$ no conteúdo de 
campos do MP. Neste capítulo obtemos os operadores de dimensão $d=5$ e $d=6$ gerados nos três modelos tipo seesaw. No caso do modelo tipo I tem-se apenas um operador de dimensão $d=6$ que modifica os acoplamentos dos neutrinos com o bóson $Z$. Além disso, aparece uma matriz $N$ não unitária que substitui a habitual matriz unitária $U_{P M N S}$ na corrente carregada. No caso do modelo tipo II são identificados três operadores de dimensão $d=6$ além de um operador de dimensão $d=4$. Estes operadores modificam o potencial do Higgs, o qual gera um deslocamento no valor esperado de vácuo (VEV) do Higgs. Além disso, há novas contribuições para a massa dos bósons de gauge, bem como o aparecimento de processos que permitem a violação de sabor, tal com $\mu \rightarrow 3 e$. No caso do modelo seesaw tipo III tem-se apenas um operador de dimensão $d=6$, que como no caso do modelo tipo I modifica os acoplamentos dos neutrinos com o bóson $Z$ e modifica a corrente carregada por meio de uma matriz não unitária $N$. Mais ainda, há a geração de uma mistura de sabor dos léptons carregados com o bóson $Z$ a qual permite gerar processos do tipo $\mu \rightarrow 3 e$ como no caso do modelo tipo II. Por último, os três modelos modificam a constante de Fermi com respeito à sua definição no contexto do MP.

No quarto capítulo estudamos a fenomenologia dos modelos mencionados no capítulo anterior, impondo limites sobre os parâmetros próprios de cada modelo (novos acoplamentos de Yukawa, massas das partículas pesadas), em função da massa dos neutrinos e razões de larguras para processos como $\mu \rightarrow 3 e$.

No quinto capítulo estuda-se a implementação do modelo de violação mínima de sabor no contexto do modelo seesaw tipo III, no qual se considera só a adição de dois tripletos de férmions implicando dois neutrinos de massa não nula. Neste contexto a informação da estrutura de sabor (coeficiente do operador $d=6$ ) é fornecida pelas massas dos neutrinos e os parâmetros de mistura. Por último, no sexto capítulo fornecemos nossas conclusões. 


\section{Capítulo 2}

\section{Modelo Padrão}

O Modelo Padrão (MP) [1] descreve as interações forte, eletromagnética e fraca das partículas elementares no contexto da Teoria Quântica de Campos. O MP é uma teoria baseada no grupo de simetria local $S U(3)_{C} \times S U(2)_{L} \times$ $U(1)_{Y}$, onde os índices $C, L$ e $Y$ denotam cor, quiralidade de mão esquerda e hipercarga fraca, respectivamente. O grupo de gauge unicamente determina as interações e o número de bósons vetoriais de gauge que correspondem aos geradores do grupo. São oito glúons não massivos, correspondentes aos oito geradores de $S U(3)_{C}$, que são os mediadores da interação forte, quatro bósons de gauge, dos quais três são massivos $\left(W^{ \pm}\right.$e $Z$ ) e um não massivo ( $\gamma$, o fóton), correspondentes aos três geradores do $S U(2)_{L}$ e um gerador de $U(1)_{Y}$, responsáveis pelas interações fracas.

\subsection{Modelo das interações eletrofracas}

Em 1967-68 Weinberg e Salam completaram um programa de unificação de gauge das interações fraca e eletromagnética, a chamada interação eletrofraca, baseada no grupo $S U(2)_{L}$ de isospin fraco, e no grupo unitário $U(1)_{Y}$ de hipercarga fraca. Glashow, em 1961, já tinha sugerido que as interações fraca e eletromagnética admitiam um grupo de simetria mais amplo que aquele de isospin: o $S U(2)_{L} \times U(1)_{Y}$, onde a hipercarga leptônica $(Y)$ está relacionada com o isospin $(T)$ e a carga elétrica $(Q)$ pela fórmula de GellMann-Nishijima:

$$
Q=T_{3}+\frac{Y}{2} .
$$


Mas no modelo de Glashow as massas dos bósons vetoriais mediadores da interação fraca eram introduzidas a mão. O mérito de Weinberg e Salam foi ter invocado a quebra espontânea da simetria de modo que os bósons vetoriais adquirissem massa através do mecanismo de Higgs [11]. Em 1971`t Hooft mostrou que a teoria era renormalizável [10], consolidando o modelo de Glashow -Weinberg - Salam das interações eletrofracas.

O grupo de gauge do modelo eletrofraco do MP é o $S U(2)_{L} \times U(1)_{Y}$, com os geradores satisfazendo a seguinte álgebra de grupo:

$$
\left[T^{i}, T^{j}\right]=i \epsilon^{i j k} T^{j}, \quad\left[T^{i}, Y\right]=0 .
$$

A lagrangiana eletrofraca invariante sob a simetria local desse grupo escrita em termos dos campos de férmions, os bósons de gauge e o dubleto de Higgs para as três gerações de léptons e quarks é dada por

$$
\mathcal{L}=\mathcal{L}_{\text {Léptons }}+\mathcal{L}_{\text {Quarks }}+\mathcal{L}_{\text {Higgs }}+\mathcal{L}_{\text {Gauge }}+\mathcal{L}_{\text {Yukawa }}
$$

A lagrangiana dos bósons de gauge tem dois termos,

$$
\mathcal{L}_{\text {Gauge }}=-\frac{1}{4} W_{\mu \nu}^{i} W^{i \mu \nu}-\frac{1}{4} B_{\mu \nu} B^{\mu \nu} .
$$

O primeiro termo é associado aos geradores de $S U(2)$ e o segundo associado ao gerador de $U(1)$, onde

$$
W_{\mu \nu}^{i}=\partial_{\mu} W_{\nu}^{i}-\partial_{\nu} W_{\mu}^{i}+g \epsilon^{i j k} W_{\mu}^{j} W_{\nu}^{k}
$$

$\mathrm{e}$

$$
B_{\mu \nu}=\partial_{\mu} B_{\nu}-\partial_{\nu} B_{\mu}
$$

A lagrangiana para os léptons do MP pode ser dividida em duas partes; os campos de mão direita e mão esquerda ${ }^{1}$

$$
\mathcal{L}_{\text {Léptons }}=\sum_{\alpha}\left[i{\overline{L^{\prime}}}_{\alpha L} \not D L^{\prime}{ }_{\alpha L}+i{\overline{l^{\prime}}}_{\alpha R} \not D l^{\prime}{ }_{\alpha R}\right] .
$$

onde

$$
L_{\alpha L}^{\prime}=\left(\begin{array}{c}
\nu^{\prime}{ }_{\alpha L} \\
l^{\prime}{ }_{\alpha L}
\end{array}\right), \quad(\alpha: e, \mu, \tau)
$$

\footnotetext{
${ }^{1}$ No MP, assume-se que os campos dos neutrinos só tem componentes de mão esquerda, implicando que os neutrinos tenham massa nula.
} 
As derivadas covariantes para os campos de mão esquerda e mão direita são dadas por

$$
\begin{aligned}
i{\overline{L^{\prime}}}_{\alpha L} \not D L_{\alpha L} & =i{\overline{L^{\prime}}}_{\alpha L} \gamma^{\mu}\left(\partial_{\mu}-i \frac{g}{2} \tau^{i} W_{\mu}^{i}-i g^{\prime} Y_{L} B_{\mu}\right) L_{\alpha L}^{\prime} \\
i{\overline{l^{\prime}}}_{\alpha R} \not D l^{\prime}{ }_{\alpha R} & =i{\overline{L^{\prime}}}_{\alpha R} \gamma^{\mu}\left(\partial_{\mu}-i g^{\prime} Y_{R} B_{\mu}\right) l_{\alpha R}^{\prime}
\end{aligned}
$$

onde $g$ e $g^{\prime}$ são duas constantes de acoplamento independentes, $g$ associada com o grupo $S U(2)_{L}, g^{\prime}$ associada com o grupo $U(1)_{Y}$ e $\tau^{i}$ são as matrizes de Pauli. Usando (2.8) na equação (2.7) com $Y_{L}=-1, Y_{R}=-2$ e omitindo os termos cinéticos, obtemos a lagrangiana de interação que descreve o acoplamento dos léptons com os bósons de gauge,

$$
\mathcal{L}_{I, L}=\frac{1}{2}\left(\begin{array}{ll}
{\overline{\nu^{\prime}}}_{\alpha L} & {\overline{l^{\prime}}}_{\alpha L}
\end{array}\right)\left(\begin{array}{cc}
g W_{3}-g^{\prime} \beta & g\left(W_{1}-i W_{2}\right) \\
g\left(W_{1}+i W_{2}\right) & -g W_{3}-g^{\prime} \not B
\end{array}\right)\left(\begin{array}{c}
\nu^{\prime}{ }_{\alpha L} \\
l^{\prime}{ }_{\alpha L}
\end{array}\right)-\overline{l^{\prime}{ }_{\alpha R}} B B l^{\prime}{ }_{\alpha R} .
$$

Pode-se separar esta lagrangiana de interação em uma lagrangiana de corrente carregada,

$$
\mathcal{L}_{I, L}^{(C C)}=\frac{g}{2}\left[\overline{\nu^{\prime}}{ }_{\alpha L}\left(W_{1}-i W_{2}\right) l^{\prime}{ }_{\alpha L}+\bar{l}^{\prime}{ }_{\alpha L}\left(W_{1}+i W_{2}\right) \nu^{\prime}{ }_{\alpha L}\right],
$$

que é dada pelos termos fora da diagonal de (2.9), e em uma lagrangiana de corrente neutra,

$$
\mathcal{L}_{I, L}^{(N C)}=\frac{g}{2}\left[{\overline{\nu^{\prime}}}_{\alpha L}\left(g W_{3}-g^{\prime} \beta\right) \nu_{\alpha_{L}}^{\prime}-\bar{l}^{\prime}{ }_{\alpha L}\left(g W_{3}+g^{\prime} \beta\right) l^{\prime}{ }_{\alpha L}-2{\overline{l^{\prime}}}_{\alpha R} B l^{\prime}{ }_{\alpha R}\right],
$$

dada pelos termos da diagonal de (2.9). Definindo

$$
W^{\mu \pm}=\frac{W_{1}^{\mu} \mp i W_{2}^{\mu}}{\sqrt{2}}
$$

na equação (2.10) obtemos

$$
\begin{aligned}
\mathcal{L}_{I, L}^{(C C)} & =\frac{g}{\sqrt{2}}\left(\overline{\nu^{\prime}}{ }_{\alpha L} W^{+} l^{\prime}{ }_{\alpha L}+{\overline{l^{\prime}}}_{\alpha L} W^{-} \nu^{\prime}{ }_{\alpha L}\right) \\
& =\frac{g}{2 \sqrt{2}} \bar{l}^{\prime} \gamma^{\mu}\left(1-\gamma^{5}\right) \nu^{\prime}{ }_{\alpha} W_{\mu}^{-}+h . c \\
& =\frac{g}{2 \sqrt{2}} J_{W L}^{\mu} W_{\mu}^{-}+h . c
\end{aligned}
$$


onde $J_{W L}^{\mu}$ é a corrente carregada dos léptons. As lagrangianas (2.11) e (2.12), não descrevem todas as interações fundamentais do MP, já que todas as partículas, bósons de gauge $\left(B^{\nu}, W^{\nu}\right)$ e férmions $(\psi)$ ainda permanecem sem massa, isto porque os termos de massa como

$$
\begin{array}{r}
\bar{\psi} \psi=\overline{\psi_{L}} \psi_{R}+\overline{\psi_{R}} \psi_{L} \\
W^{\nu} W_{\nu} \text { e } B^{\nu} B_{\nu}
\end{array}
$$

quebram a invariância de gauge. Para entender isto, tem-se que lembrar que a lagrangiana $\mathcal{L}_{\text {Gauge }}$ é invariante sob

$$
\begin{aligned}
W_{\nu}^{i} \tau_{i} / 2 & \rightarrow U\left(\theta^{i}(x)\right)\left[W_{\nu}^{i} \tau_{i} / 2+\frac{1}{g} \partial_{\nu}\right] U^{-1}\left(\theta^{i}(x)\right), \\
B_{\nu} Y / 2 & \rightarrow U(\alpha(x))\left[B_{\nu} Y / 2+\frac{1}{g^{\prime}} \partial_{\nu}\right] U^{-1}(\alpha(x)) .
\end{aligned}
$$

onde

$$
U\left(\theta^{i}(x)\right)=e^{i \theta^{i}(x) \tau_{i} / 2}, \quad U(\alpha(x))=e^{i \alpha(x) Y / 2} .
$$

Assim, o termo de massa para $B^{\nu}$ que é dado por $B^{\nu} B_{\nu}$ não é invariante de gauge, porque

$$
B^{\nu} B_{\nu} \stackrel{U(\alpha(x))}{\rightarrow}\left(B^{\nu}+\frac{i}{g^{\prime}} \partial^{\nu} \alpha(x)\right)\left(B^{\nu}+\frac{i}{g^{\prime}} \partial_{\nu} \alpha(x)\right) \neq B^{\nu} B_{\nu}
$$

Portanto, para obter termos que gerem massa e sejam invariantes de gauge, se tem que introduzir o mecanismo de Higgs.

\subsubsection{O Mecanismo de Higgs}

No MP, a massa dos bósons de gauge, como dos férmions, são geradas pelo mecanismo de Higgs [11] através do dubleto de Higgs

$$
\Phi(x)=\left(\begin{array}{c}
\Phi^{+}(x) \\
\Phi^{0}(x)
\end{array}\right),
$$

onde $\Phi^{+}(x)$ é o campo escalar complexo carregado e $\Phi^{0}(x)$ é o campo escalar complexo neutro. A lagrangiana de Higgs invariante sob a simetria local $S U(2)_{L} \times U(1)_{Y}$ é:

$$
\mathcal{L}_{\text {Higgs }}=\left(D_{\mu} \Phi\right)^{\dagger}\left(D^{\mu} \Phi\right)-\mu^{2} \Phi^{\dagger} \Phi-\lambda\left(\Phi^{\dagger} \Phi\right)^{2},
$$


onde o coeficiente $\lambda$ é positivo, para que o potencial

$$
V_{\text {Higgs }}(x)=\mu^{2} \Phi^{\dagger} \Phi+\lambda\left(\Phi^{\dagger} \Phi\right)^{2},
$$

seja limitado inferiormente. Por outro lado, o quadrado $\mu^{2}$ é assumido negativo, para realizar a quebra espontânea da simetria [11],

$$
S U(2)_{L} \times U(1)_{Y} \Rightarrow U(1)_{Q},
$$

onde $U(1)_{Q}$ é o grupo de simetria de gauge das interações eletromagnéticas, associado com a conservação da carga elétrica que, como é bem conhecido, não é quebrada. Definindo

$$
v \equiv \sqrt{-\frac{\mu^{2}}{\lambda}},
$$

o potencial de Higgs na equação (2.20) pode ser escrito como

$$
V_{\text {Higgs }}(x)=\lambda\left(\Phi^{\dagger} \Phi-\frac{v^{2}}{2}\right)^{2}+\frac{\lambda v^{4}}{4}-\lambda \frac{v^{4}}{4},
$$

desta expressão fica evidente que o potencial é mínimo para

$$
\Phi^{\dagger} \Phi=\frac{v^{2}}{2} .
$$

Em teoria quântica de campos o mínimo do potencial corresponde ao vácuo, que é o estado de menor energia, e as excitações quânticas de cada campo sobre o vácuo correspondem aos estados das partículas. Um campo escalar neutro pode ter um valor esperado no vácuo (VEV) não nulo. Da equação (2.24) se pode observar que o campo Higgs tem um VEV não nulo. Para ter um vácuo eletricamente neutro, o VEV para o campo de Higgs deve ser

$$
\langle\Phi\rangle=\frac{1}{\sqrt{2}}\left(\begin{array}{l}
0 \\
v
\end{array}\right) .
$$

A simetria $S U(2)_{L} \times U(1)_{Y}$ é espontaneamente quebrada pelo $\mathrm{VEV}<\Phi>$

$$
T_{1}\langle\Phi\rangle \neq 0, \quad T_{2}\langle\Phi\rangle \neq 0, \quad T_{3}\langle\Phi\rangle \neq 0, \quad Y\langle\Phi\rangle \neq 0(2.26)
$$

mas

$$
Q\langle\Phi\rangle=0,
$$


o que faz com que o fóton permaneça sem massa. O campo de Higgs pode ser parametrizado como

$$
\Phi(x)=\frac{1}{\sqrt{2}} e^{\frac{i}{2 v} \xi^{i}(x) \cdot t^{i}}\left(\begin{array}{c}
0 \\
v+h(x)
\end{array}\right)=\frac{1}{\sqrt{2}}\left(\begin{array}{c}
\sqrt{2} \phi^{+} \\
v+h(x)+i \eta
\end{array}\right),
$$

onde $\phi^{ \pm}$e $\eta$ são os bósons de Goldstone. Agora se pode escolher um gauge no qual $\Phi(x)$ se escreve como

$$
\Phi(x)=\frac{1}{\sqrt{2}}\left(\begin{array}{c}
0 \\
v+h(x)
\end{array}\right),
$$

e

$$
\begin{aligned}
D_{\mu} \Phi(x) & =\left[\partial_{\mu}-i \frac{g}{2} \tau^{i} W_{\mu}^{i}-\frac{i}{2} g^{\prime} B_{\mu}\right] \Phi(x) \\
& =\frac{1}{\sqrt{2}}\left(\begin{array}{c} 
\\
\quad-\frac{i g}{\sqrt{2}} W_{\mu}^{-}(x)[v+h(x)] \\
\partial_{\mu} h(x)+\frac{i}{2}\left(g W_{\mu}^{3}(x)-g^{\prime} B_{\mu}(x)\right)[v+h(x)]
\end{array}\right),
\end{aligned}
$$

tal gauge é chamado de gauge unitário. Neste gauge, a lagrangiana de Higgs de (2.19) é dada por:

$$
\begin{aligned}
\mathcal{L}_{\text {Higgs }} & =\frac{1}{2} \partial_{\mu} h \partial^{\mu} h+\frac{g^{2}}{4}(v+h)^{2} W_{\mu}^{-} W^{+\mu}+\lambda \frac{v^{4}}{4} \\
& +\frac{1}{8}(v+h)^{2}\left(g W_{\mu}^{3}-g^{\prime} B_{\mu}\right)^{2}-\frac{\lambda}{4}\left(h^{2}+2 v h\right)^{2} .
\end{aligned}
$$

Na equação (2.31) se pode identificar um termo de massa da forma

$$
\begin{aligned}
\mathcal{L}_{\text {massa }} & =\frac{1}{2} \frac{v^{2}}{4}\left(g W_{\mu}^{3}-g^{\prime} B_{\mu}\right)^{2} \\
& =\frac{1}{2}\left(\begin{array}{ll}
W_{\mu}^{3} & B_{\mu}
\end{array}\right) \frac{v^{2}}{4}\left(\begin{array}{cc}
g^{2} & -g g^{\prime} \\
-g g^{\prime} & g^{2}
\end{array}\right)\left(\begin{array}{c}
W^{3 \mu} \\
B^{\mu}
\end{array}\right),
\end{aligned}
$$

diagonalizando este termo de massa, se obtém os auto estados e auto valores de massa, fornecidos por

$$
\begin{array}{ll}
Z_{\mu}=\frac{g W_{\mu}^{3}-g^{\prime} B_{\mu}}{\sqrt{g^{2}+g^{\prime 2}}}, \text { com } & M_{Z}=\frac{v \sqrt{g^{2}+g^{\prime 2}}}{2}, \\
A_{\mu}=\frac{g W_{\mu}^{3}+g^{\prime} B_{\mu}}{\sqrt{g^{2}+g^{\prime 2}}}, \text { com } & M_{A}=0 .
\end{array}
$$


Expandindo a equação (2.31) e usando (2.33), obtemos:

$$
\begin{aligned}
\mathcal{L}_{\text {Higgs }} & =\frac{1}{2} \partial_{\mu} h \partial^{\mu} h-\lambda v^{2} h^{2}+\frac{g^{2} v^{2}}{4} W_{\mu}^{-} W^{+\mu}+\frac{\left(g^{2}+g^{\prime 2}\right) v^{2}}{8} Z_{\mu} Z^{\mu} \\
& -\lambda v^{2} h^{3}-\frac{\lambda}{4} h^{4}+\lambda \frac{v^{4}}{4} \frac{g^{2} v}{2} W_{\mu}^{-} W^{+\mu} h+\frac{\left(g^{2}+g^{\prime 2}\right) v}{4} Z_{\mu} Z^{\mu} h \\
& +\frac{g^{2}}{4} W_{\mu}^{-} W^{+\mu} h^{2}+\frac{\left(g^{2}+g^{\prime 2}\right)}{8} Z_{\mu} Z^{\mu} h^{2}
\end{aligned}
$$

O primeiro termo de (2.35) é o termo cinético para o bóson de Higgs. O segundo termo é o termo de massa para o bóson de Higgs. Assim, a massa do bóson de higgs é dada por

$$
m_{h}=\sqrt{2 \lambda v^{2}}=\sqrt{-2 \mu^{2}} .
$$

O terceiro e quarto termo de (2.35) são os termos de massa para os bósons de gauge $W^{\mu}$ e $Z^{\mu}$ respectivamente. Assim, as massas dos bósons de gauge são

$$
M_{W}=\frac{g v}{2}, \quad M_{Z}=\frac{\sqrt{g^{2}+g^{\prime 2}} v}{2} .
$$

De (2.34), podemos identificar o bóson $A_{\mu}$ de massa nula com o fóton. Como a teoria deve incluir a interação eletromagnética descrita pela lagrangiana da eletrodinâmica quântica (QED)

$$
\mathcal{L}_{I, L}^{(\gamma)}=e J_{\gamma L}^{u} A_{u}=-e \bar{l}_{\alpha} \gamma^{u} l_{\alpha} A_{u}
$$

Definindo

$$
\tan \theta_{W}=\frac{g^{\prime}}{g}
$$

onde $\theta_{W}$ é o ângulo de mistura fraco, e usando (2.33) e (2.34), a lagrangiana de corrente neutra (2.11), pode ser escrita como

$$
\begin{aligned}
\mathcal{L}_{I, L}^{(N C)} & =\frac{g}{2 \cos \theta_{W}}\left[{\overline{\nu^{\prime}}}_{\alpha L} \not Z \nu_{\alpha_{L}}^{\prime}-{\overline{l^{\prime}}}_{\alpha L} \not Z l^{\prime}{ }_{\alpha L}+2 \sin ^{2} \theta_{W}{\overline{l^{\prime}}}_{\alpha} \not Z l^{\prime}{ }_{\alpha}\right] \\
& -g \sin \theta_{W}{\overline{l^{\prime}}}_{\alpha} A l^{\prime}{ }_{\alpha} .
\end{aligned}
$$




\begin{tabular}{|c|c|c|}
\hline férmions & $g_{V}$ & $g_{A}$ \\
\hline$\nu_{e}, \nu_{\mu}, \nu_{\tau}$ & $g_{V}^{\nu}=\frac{1}{2}$ & $g_{A}^{\nu}=\frac{1}{2}$ \\
$e, \mu, \tau$ & $g_{V}^{l}=-\frac{1}{2}+2 \sin ^{2} \theta_{W}$ & $g_{A}^{l}=-\frac{1}{2}$ \\
$u, c, t$ & $g_{V}^{U}=\frac{1}{2}-\frac{4}{3} \sin ^{2} \theta_{W}$ & $g_{A}^{U}=\frac{1}{2}$ \\
$d, s, b$ & $g_{V}^{D}=-\frac{1}{2}+\frac{2}{3} \sin ^{2} \theta_{W}$ & $g_{A}^{D}=-\frac{1}{2}$ \\
\hline
\end{tabular}

Tabela 2.1: Valores de $g_{A}$ e $g_{V}$ para os férmions. As subscrições $\nu, l, U, D$, indicam respectivamente um neutrino, lépton carregado, quark do tipo up e quark do tipo down.

Como o último termo de (2.40) fornece o acoplamento dos campos dos léptons com o campo eletromagnético, ele deve coincidir com a lagrangiana de interação da QED dada pela equação (2.38) e assim encontramos

$$
g \sin \theta_{W}=e .
$$

Esta é uma importante relação, que conecta a constante $g$ com o ângulo $\theta_{W}$. A lagrangiana de corrente neutra agora pode ser escrita como

$$
\mathcal{L}_{I, L}^{(N C)}=\mathcal{L}_{I, L}^{(Z)}+\mathcal{L}_{I, L}^{(\gamma)}
$$

onde $\mathcal{L}_{I, L}^{\gamma}$ é a lagrangiana de interação da QED dada pela equação (2.38) e $\mathcal{L}_{L}^{Z}$ é lagrangiana de corrente neutra fraca dada por

$$
\mathcal{L}_{I, L}^{(Z)}=\frac{g}{2 \cos \theta_{W}} J_{Z, L}^{\mu} Z_{\mu}
$$

com a corrente neutra fraca dos léptons

$$
J_{Z, L}^{\mu}={\overline{\nu^{\prime}}}_{\alpha} \gamma^{\mu}\left(g_{V}^{\nu}-g_{A}^{\nu} \gamma^{5}\right) \nu_{\alpha}^{\prime}+{\overline{l^{\prime}}}_{\alpha} \gamma^{u}\left(g_{V}^{\alpha}-g_{A}^{\alpha} \gamma^{5}\right) l_{\alpha}^{\prime},
$$

onde introduz-se os coeficientes

$$
g_{V}^{\alpha}=T_{3}^{\alpha}-2 q_{\alpha} \sin ^{2} \theta_{W}, \quad g_{A}^{\alpha}=T_{3}^{\alpha}
$$

cujos valores, obtidos de (2.40), são dadas na tabela (2.1) onde $T_{3}^{\alpha}$ é o valor da terceira componente do isospin fraco e $q_{\alpha}$ é a carga elétrica dos léptons em unidades de carga elétrica $e^{2}$.

\footnotetext{
${ }^{2} \mathrm{O}$ índice $\alpha$ indica um férmion.
} 
Para obter os valores teóricos das massas dos bósons $Z_{\mu}$ e $W_{\mu}$ precisamos conhecer as constantes de acoplamento e o VEV do higgs. Usando a relação

$$
\frac{g}{2 \sqrt{2}}=\left[\frac{M_{W}^{2} G_{F}}{\sqrt{2}}\right]^{1 / 2},
$$

e substituindo $M_{W}$ nesta equação, encontramos

$$
v=\left[\sqrt{2} G_{F}\right]^{-1 / 2} \simeq 246 \mathrm{GeV} .
$$

Usando o valor experimental do $\sin ^{2} \theta_{W} \simeq 0,23$ [22], na equação (2.41), se obtém

$$
g=\frac{e}{\sin \theta_{W}} \simeq 0.65 .
$$

Com esses valores em (2.37) obtemos

$$
\begin{aligned}
M_{W} & \simeq 80 \mathrm{GeV}, \\
M_{Z} & =\frac{M_{W}}{\cos \theta_{W}} \simeq 91 \mathrm{GeV} .
\end{aligned}
$$

Esses valores estão de acordo com os dados experimentais $M_{w}=80,385 \pm$ 0, 015GeV, $M_{Z}=91,1876 \pm 0,0021 \mathrm{GeV}$, [22], assim como a previsão para o parâmetro $\rho$ a nível árvore,

$$
\rho=\frac{M_{W}^{2}}{\cos ^{2} \theta_{W} M_{Z}^{2}}=1,
$$

com a incorporação das correções de ordem mais altas, este valor é modificado ligeiramente e se torna compatível com resultados experimentais de lata precisão [22], que demonstram os resultados bem sucedidos do MP.

\section{Interações eletrofracas para os quarks}

Assim como os léptons, os quarks são partículas elementares que podem sentir as interações fraca e eletromagnética, e sendo assim podemos ampliar o modelo eletrofraco a essas partículas.

Os quarks podem ser introduzidos no MP de forma semelhante aos léptons. A lagrangiana para os quarks sem massa é dada por

$$
\mathcal{L}_{\text {Quarks }}=i{\overline{Q^{\prime}}}_{\alpha L} \not D Q_{\alpha L}^{\prime}+i{\overline{q^{\prime}}}_{\alpha R}^{D} \not D{q^{\prime}}_{\alpha R}^{D}+i{\overline{q^{\prime}}}_{\alpha R}^{U} \not D{q^{\prime}}_{\alpha R}^{U},
$$


onde

$$
Q_{1 L}^{\prime}=\left(\begin{array}{c}
u^{\prime}{ }_{L} \\
d^{\prime}{ }_{L}
\end{array}\right), \quad Q_{2 L}^{\prime}=\left(\begin{array}{c}
c^{\prime}{ }_{L} \\
s_{L}^{\prime}
\end{array}\right), \quad Q_{3 L}^{\prime}=\left(\begin{array}{c}
t_{L}^{\prime} \\
b_{L}^{\prime}
\end{array}\right)
$$

e

$$
\begin{array}{ll}
q_{\alpha R}^{\prime U}=\alpha_{R}^{\prime}, & (\alpha: u, c, t), \\
q_{\beta R}^{\prime D}=\beta_{R}^{\prime}, & (\beta: d, s, b) .
\end{array}
$$

Os acoplamentos com os bósons de gauge vêm da derivada covariante,

$$
\begin{aligned}
& L \quad: \quad D_{\mu} \rightarrow \partial_{\mu}-i \frac{g}{2} \tau^{i} W_{\mu}^{i}-i g^{\prime} Y_{L} B_{\mu}, \\
& R \quad: \quad D_{\mu} \rightarrow \partial_{\mu}-i g^{\prime} Y_{R} B_{\mu} .
\end{aligned}
$$

As hipercargas para os quarks podem ser obtidas pela fórmula de Gell-MannNishijima (2.41), conhecida a carga $Q$ e a terceira componente de isospin $T_{3}$, Usando os valores das hipercargas para os quarks na lagrangiana dos quarks podemos obter as correntes carregadas e neutras,

$$
\begin{gathered}
\mathcal{L}_{I, Q}^{(C C)}=\frac{g}{2 \sqrt{2}} \overline{q^{\prime}{ }_{\alpha}^{U}} \gamma^{\mu}\left(1-\gamma^{5}\right) q_{\alpha}^{\prime D} W_{\mu}^{-}+h . c \\
=\frac{g}{2 \sqrt{2}} J_{W Q}^{\mu} W_{\mu}^{-}+h . c \\
\mathcal{L}_{I, Q}^{(N C)}=\frac{g}{2 \cos \theta_{W}}\left[\overline{q^{\prime}}{ }_{\alpha} \gamma^{\mu}\left(g_{V}^{U}-g_{A}^{U} \gamma^{5}\right) q_{\alpha}^{\prime U}+\overline{{q^{\prime}}_{\alpha}^{D}} \gamma^{\mu}\left(g_{V}^{D}-g_{A}^{D} \gamma^{5}\right) q_{\alpha}^{\prime D}\right] Z_{\mu}, \\
=\frac{g}{2 \cos \theta_{W}} J_{Z Q}^{\mu} Z_{\mu} .
\end{gathered}
$$

onde os valores de $g_{V}^{\alpha}$ e $g_{A}^{\alpha}$, são dados na tabela (2.1).

\subsubsection{Massa dos férmions e mistura}

No MP, a massa dos férmions é resultado do mecanismo de Higgs através da presença dos acoplamentos de Yukawa dos campos dos férmions com o dubleto de Higgs. Considere-se primeiro a massa dos léptons, a lagrangiana Yukawa-lépton-Higgs invariante sob $S U(2)_{L} \times U(1)_{Y}$ é:

$$
\mathcal{L}_{H L}=-Y_{\alpha \beta}^{\prime l}{\overline{L^{\prime}}}_{\alpha L} \Phi l^{\prime}{ }_{\beta R}+h . c,
$$


onde a matriz $Y^{\prime l}$ de acoplamento de Yukawa é, em geral, uma matriz complexa. No gauge unitário, o dubleto de Higgs tem a forma dada pela equação (2.29) e a lagrangiana Yukawa-lépton-Higgs na equação (2.58) transforma-se em

$$
\mathcal{L}_{H L}=-\left(\frac{v+h}{\sqrt{2}}\right) Y_{\alpha \beta}^{\prime l} \overline{l_{\alpha L}^{\prime}} l_{\beta R}^{\prime}+\text { h.c. }
$$

O termo proporcional ao VEV, $v$, do dubleto de Higgs é o termo de massa para os férmions carregados. Desde que a matriz $Y^{\prime l}$ seja em geral não diagonal, os campos não têm massa definida. Para encontrar léptons carregados com massas definidas, é necessário diagonalizar a matriz $Y^{\prime l}$. A matriz $Y^{\prime l}$ pode ser diagonalizada através da transformação biunitária

$$
V_{L}^{l \dagger} Y^{\prime l} V_{R}^{l}=Y^{l}, \operatorname{com} \quad Y_{\alpha \beta}^{l}=y_{\alpha}^{l} \delta_{\alpha \beta},
$$

onde $V_{L}^{l}$ e $V_{R}^{l}$ são matrizes unitárias. A diagonalização em (2.59) leva

$$
\mathcal{L}_{H L}=-\left(\frac{v+h}{\sqrt{2}}\right) \overline{l_{L}} Y^{l} l_{R}+\text { h.c. }
$$

onde

$$
l_{L}=V_{L}^{l \dagger} l_{\alpha L}^{\prime}, \quad l_{R}=V_{R}^{l \dagger} l^{\prime}{ }_{\alpha R}
$$

são as componentes de mão esquerda e direita, respectivamente, dos campos de léptons carregados com massa definida. Assim, a lagrangiana Yukawalépton-Higgs pode ser escrita como

$$
\mathcal{L}_{H L}=-\frac{y_{\alpha}^{l} v}{\sqrt{2}} \overline{l_{\alpha}} l_{\alpha}-\frac{y_{\alpha}^{l}}{\sqrt{2}} \overline{l_{\alpha}} l_{\alpha} h
$$

com

$$
l_{\alpha}=l_{\alpha L}+l_{\alpha R}
$$

O primeiro termo de (2.63) é o termo de massa para os léptons carregados, cujas massas são dadas por

$$
m_{\alpha}=\frac{y_{\alpha}^{l} v}{\sqrt{2}}
$$


Como resultado da transformação dada por (2.62), a corrente carregada para léptons na equação (2.12) pode ser escrita como:

$$
j_{W, L}^{\mu}=2 \bar{l}^{\prime}{ }_{L} \gamma^{\mu} \nu_{L}^{\prime}=2 \bar{l}_{L} \gamma^{\mu} V_{L}^{l \dagger} \nu_{L}^{\prime} .
$$

Uma vez que é possível transformar livremente os campos de neutrinos não massivos

$$
\nu_{\alpha L}=V_{L}^{l \dagger} \nu_{L}^{\prime} \quad(\alpha=e, \mu, \tau),
$$

a corrente carregada para os léptons pode ser escrita como

$$
j_{W, L}^{\mu}=2 \bar{l}_{\alpha L} \gamma^{\mu} \nu_{\alpha L}
$$

em termo dos campos de neutrinos não massivos $\nu_{e}, \nu_{\mu}, \nu_{\tau}$ e os campos de léptons carregados massivos definidos em (2.62). Os campos $\nu_{e}, \nu_{\mu}, \nu_{\tau}$ são chamados campos de neutrinos de sabor.

Considera-se agora a lagrangiana com termos de massa para os quarks. Como para os léptons, procura-se o produto de campos de mão direita com campos de mão esquerda. Dos dubletos de mão esquerda em (2.52) e os singletos de mão direita em (2.53) e (2.54), é possível formar dois produtos do seguinte tipo:

$$
\begin{aligned}
& \overline{Q_{k L}^{\prime}} q_{\beta R}^{\prime D}, \quad \text { com } \quad(k=1,2,3) \quad \text { e } \quad(\beta=d, s, b) . \\
& \overline{Q^{\prime}{ }_{k L}} q_{\beta R}^{\prime}, \quad \text { com } \quad(k=1,2,3) \quad \text { e } \quad(\beta=u, c, t) .
\end{aligned}
$$

O produto em (2.69) tem $Y=-1$ e pode se acoplar com o dubleto de Higgs com $Y=+1$, para ter um termo da lagrangiana de Yukawa invariante sob $S U(2)_{L} \times U(1)_{Y}$, o produto em (2.70) tem $Y=+1$. Assim, para gerar um termo de massa precisamos de um dubleto de Higgs com $Y=-1$. Partindo de (2.18), define-se o dubleto conjugado, que é um dubleto de $S U(2)$, da seguinte forma

$$
\tilde{\Phi}=i \tau_{2} \Phi^{*}
$$

Isso permite escrever a lagrangiana de Yukawa-quarks-Higgs invariante sob $S U(2)_{L} \times U(1)_{Y}$ como

$$
\mathcal{L}_{H Q}=-\sum_{k=1,2,3}\left[\sum_{\beta=d, s, b} Y_{k \beta}^{\prime D} \overline{Q_{k L}^{\prime}} \Phi q_{\beta R}^{\prime D}+\sum_{\beta=u, c, t} Y_{k \beta}^{\prime U} \overline{Q_{k L}^{\prime}} \tilde{\Phi} q_{\beta R}^{\prime U}\right]+\text { h.c. }
$$


No gauge unitário, a lagrangiana Yukawa-quarks-Higgs na equação (2.72) transforma-se

$$
\mathcal{L}_{H Q}=-\left(\frac{v+h}{\sqrt{2}}\right)\left[\sum_{\alpha, \beta=d, s, b} Y_{\alpha \beta}^{\prime D} \overline{q^{\prime D}}{ }_{\alpha L}^{\prime D}{ }_{\beta R}+\sum_{\alpha, \beta=u, c, t} Y_{\alpha \beta}^{\prime U} \overline{q_{\alpha L}^{\prime U}} q_{\beta R}^{\prime U}\right]+\text { h.c. }(2 .
$$

Os termos proporcionais a $v$ são os termos de massa para os quarks. Como as matrizes $Y^{\prime D}$ e $Y^{\prime U}$ são em geral não diagonais, os campos de quarks linha não têm massa definida. Para encontrar auto-estados de massa, é necessário diagonalizar as matrizes $Y^{\prime D}$ e $Y^{\prime U}$. Usando o mesmo procedimento, como no caso dos léptons, as matrizes $Y^{\prime D}$ e $Y^{\prime U}$ podem ser diagonalizadas através das transformações biunitárias

$$
\begin{aligned}
& V_{L}^{D \dagger} Y^{\prime D} V_{R}^{D}=Y^{D}, \quad \text { com } \quad Y_{\alpha \beta}^{D}=y_{\alpha}^{D} \delta_{\alpha \beta} \\
& V_{L}^{U \dagger} Y^{\prime U} V_{R}^{U}=Y^{U}, \quad \text { com } \quad Y_{\alpha \beta}^{U}=y_{\alpha}^{U} \delta_{\alpha \beta} .
\end{aligned}
$$

A diagonalização em (2.73) leva

$$
\mathcal{L}_{H Q}=-\left(\frac{v+h}{\sqrt{2}}\right)\left[\overline{q_{L}} Y^{D} q_{R}^{D}+\overline{q_{L}} Y^{U} q_{R}^{U}\right]+h . c
$$

onde

$$
q_{L}^{U}=V_{L}^{U \dagger} q_{L}^{\prime U}, \quad q_{R}^{U}=V_{R}^{U \dagger} q_{\alpha R}^{\prime U}, \quad q_{L}^{D}=V_{L}^{D \dagger} q_{\alpha L}^{\prime D}, \quad q_{R}^{D}=V_{R}^{D \dagger} q_{\alpha R}^{D}
$$

são as componentes de mão direita e esquerda dos campos dos quarks com massa definida. Assim, a lagrangiana Yukawa-quarks-Higgs pode ser escrita como

$$
\begin{aligned}
\mathcal{L}_{H Q} & =-\sum_{\alpha=d, s, b} \frac{y_{\alpha}^{D} v}{\sqrt{2}} \overline{q_{\alpha}^{D}} q_{\alpha}^{D}-\sum_{\alpha=u, c, t} \frac{y_{\alpha}^{U} v}{\sqrt{2}} \overline{q_{\alpha}^{U}} q_{\alpha}^{U} \\
& -\sum_{\alpha=d, s, b} \frac{y_{\alpha}^{D}}{\sqrt{2}} \overline{q_{\alpha}^{D}} q_{\alpha}^{D} h-\sum_{\alpha=u, c, t} \frac{y_{\alpha}^{U}}{\sqrt{2}} \overline{q_{\alpha}^{U}} q_{\alpha}^{U} h
\end{aligned}
$$

onde

$$
q_{\alpha}^{D}=q_{\alpha L}^{D}+q_{\alpha R}^{D}, \quad q_{\alpha}^{U}=q_{\alpha L}^{U}+q_{\alpha R}^{U}
$$


O primeiro e segundo termo de (2.78) são os termos de massa para os quarks, cujas massas são dadas por

$$
\begin{aligned}
& m_{\alpha}=\frac{y_{\alpha}^{D} v}{\sqrt{2}} \quad(\alpha=d, s, b), \\
& m_{\beta}=\frac{y_{\beta}^{U} v}{\sqrt{2}} \quad(\beta=u, c, t) .
\end{aligned}
$$

Agora é possível expressar os campos linha para os quarks em termos dos campos de quark massivos, usando (2.77); a definição da corrente carregada para os quarks, dada por (2.56)

$$
J_{W Q}^{\mu}=2 \overline{q_{\alpha L}^{U}} V_{L}^{U \dagger} \gamma^{\mu} V_{L}^{D} q_{\alpha L}^{D}=2 \overline{q_{\alpha L}^{U}} \gamma^{\mu} V_{C K M} q_{\alpha L}^{D}
$$

onde $V_{C K M}=V_{L}^{U \dagger} V_{L}^{D}$ é a matriz de Cabibbo-Kobayashi-Maskawa, que mistura os autoestados de massa das diferentes gerações. Por outro lado, a corrente neutra para os quarks dada por (2.57) em termos de $g_{L}^{\alpha}=\frac{g_{V}^{\alpha}+g_{V}^{\alpha}}{2} \mathrm{e}$ $g_{R}^{\alpha}=\frac{g_{V}^{\alpha}-g_{V}^{\alpha}}{2}$, pode ser escrita como

$$
\begin{aligned}
J_{Z Q}^{\mu}= & 2\left[g_{L}^{U} \overline{{q^{\prime}}^{U}}{ }_{L} \gamma^{\mu} q_{L}^{\prime U}+g_{R}^{U} \overline{q^{\prime U}}{ }_{R} \gamma^{\mu} q_{R}^{\prime U}\right. \\
& \left.+g_{L}^{D} \overline{{q^{\prime}}^{D}}{ }_{L} \gamma^{\mu} q_{L}^{\prime D}+g_{R}^{D} \overline{q^{\prime D}}{ }_{R} \gamma^{\mu} q_{R}^{\prime D}\right] \\
= & 2\left[g_{L}^{U} \overline{q^{U}}{ }_{L} V_{L}^{U \dagger} V_{L}^{U} \gamma^{\mu} q_{L}^{U}+g_{R}^{U} \overline{q^{U}}{ }_{R} V_{R}^{U \dagger} V_{R}^{U} \gamma^{\mu} q_{R}^{U}\right. \\
& \left.+g_{L}^{D} \overline{q^{D}}{ }_{L} V_{L}^{D \dagger} V_{L}^{D} \gamma^{\mu} q_{L}^{D}+g_{R}^{D} \overline{q^{D}}{ }_{R} V_{R}^{D \dagger} V_{R}^{D} \gamma^{\mu} q_{R}^{D}\right]
\end{aligned}
$$

Como $V_{L, R}^{U}$ e $V_{L, R}^{D}$, são matrizes unitárias, não ocorre mistura no setor neutro.

\subsection{Necessidade de Estender o Modelo Padrão: Não Conservação do Número leptônico e Mistura de Neutrinos}

Pouco depois que Reines e Cowel confirmarem a existência dos neutrinos [41][42], Raymond Davis começou a aventura para detectar os neutrinos que são produzidos no Sol, [46]. Raymond Davis propôs a construção de um detector subterrâneo para detectar os neutrinos solares. A maior parte dos neutrinos 
produzidos no Sol possuem energias muito baixas para serem detectadas com a tecnologia da época, o decaimento do boro no Sol,

$$
{ }^{8} B \rightarrow{ }^{8} B e^{*}+e^{+}+\nu_{e},
$$

produz neutrinos com níveis de energia detectáveis. Com a técnica criada por Bruno Pontecorvo e Luis Alvarez [14]-[15], Davis conseguiu instalar um tanque cheio com 600 toneladas de tetracloroetileno em 1967, na mina de ouro de Homestake. Os neutrinos solares eram detectados através da reação

$$
{ }^{37} \mathrm{Cl}+\nu_{e} \rightarrow{ }^{37} \mathrm{Ar}+e^{-},
$$

feito isso, Raymond Davis se deparou com uma inesperada surpresa: o número de neutrinos provenientes do Sol, estimados de acordo com seu experimento, era em torno de $35 \%$ do número esperado de neutrinos que deveriam ser detectados de acordo com o modelo padrão Solar. Ao esforço de Raymond Davis se uniu Masatoshi Koshiba em 1987 com o detector japonês chamado Kamiokande [43], que confirmou os resultados de Raymond Davis.

Em 1999, se iniciam as observações do SNO (Sudbury Neutrino Observatory), [44]. O detector, uma grande esfera rodeada por fotomultiplicadores, com aproximadamente 1000 toneladas de água pesada, $\mathrm{D}_{2} \mathrm{O}$, e localizado na mina Creighton, no Canadá. Apesar de ser um experimento como Kamiokande e Super-Kamiokande,[45], o SNO foi elaborado para ser sensível a todos os sabores de neutrinos ativos e não apenas ao $\nu_{e}$ (como o Kamiokande e Super Kamiokande). Esta sensibilidade ocorre porque os neutrinos podem interagir com a água pesada do SNO por meio de três reações,

$$
\begin{aligned}
& \nu_{e}+d \rightarrow p+p+e^{-}, \\
& \nu_{\alpha}+d \rightarrow p+n+\nu_{\alpha}, \quad(\alpha=e, \mu, \tau), \\
& \nu_{\alpha}+e^{-} \rightarrow \nu_{\alpha}+e^{-} .
\end{aligned}
$$

Em 18 de Junho do 2001 o grupo do SNO fez um anúncio dramático: o problema dos neutrinos solares foi resolvido. O grupo SNO comparou as taxas dos neutrinos obtidas na reação (2.86) com os resultados da dispersão do $\nu_{e}$ no Super Kamiokande. De acordo com o modelo padrão solar, apenas deveriam chegar neutrinos eletrônicos para a Terra, pelo qual as medidas em ambos experimentos deveriam coincidir. As taxas medidas foram diferentes, isto indicou que no fluxo de neutrinos solares existia uma componente diferente de $\nu_{e}$. Combinando as medidas do SNO e do Super Kamiokande, o 


\begin{tabular}{|cccccccccccc|}
\hline & $\nu_{e}$ & $e^{-}$ & $\nu_{\mu}$ & $\mu^{-}$ & $\bar{\nu}_{e}$ & $e^{+}$ & $\bar{\nu}_{\mu}$ & $\mu^{+}$ & $\cdots$ & hádrons & fóton \\
\hline$L_{e}$ & 1 & 1 & 0 & 0 & -1 & -1 & 0 & 0 & $\cdots$ & 0 & 0 \\
$L_{\mu}$ & 0 & 0 & 1 & 1 & 0 & 0 & -1 & -1 & $\ldots$ & 0 & 0 \\
$\vdots$ & $\vdots$ & $\vdots$ & $\vdots$ & $\vdots$ & $\vdots$ & $\vdots$ & $\vdots$ & $\vdots$ & $\vdots$ & $\vdots$ & \\
\hline
\end{tabular}

Tabela 2.2: Atribuição de número de sabor leptônico.

grupo do SNO determinou o número total de neutrinos solares de todos os sabores $(e, \mu$ e $\tau)$, verificando que este coincide com o número de neutrinos eletrônicos produzidos no Sol calculados de acordo com o modelo padrão solar. O problema estava resolvido! Os neutrinos eletrônicos em seu caminho do Sol até a Terra não desapareciam, de alguma forma mudavam de sabor. Essa forma havia sido postulada em 1957 por Pontecorvo [16]-[17] e foi chamada de oscilação de neutrinos, mas a oscilação destas partículas só acontece se eles possuírem massa.

No MP os neutrinos são não massivos e o número leptônico é estritamente conservado. O estudo de processos que violem esta conservação pode fornecer sinais de uma física além do MP. A necessidade de introduzir o número leptônico (cargas leptônicas ) foi para explicar a não observação de certos processos como

$$
\nu_{\mu}+N \rightarrow \mu^{+}+X
$$

$\mathrm{Ou}$

$$
\bar{\nu}_{\mu}+N \rightarrow \mu^{-}+X
$$

já que processos como

$$
\nu_{\mu}+N \rightarrow \mu^{-}+X
$$

$\mathrm{Ou}$

$$
\bar{\nu}_{\mu}+N \rightarrow \mu^{+}+X
$$

eram vistos.

A explicação convencional baseia-se nas duas hipóteses seguintes: 
- $\nu_{\mu}$ e $\bar{\nu}_{\mu}$ são distintos um do outro.

- Há um número quântico que deve ser conservado na interação, cujas atribuições são fornecidas na tabela (1.2).

\subsubsection{Neutrino de Dirac e Majorana}

Agora estudaremos as características dos neutrinos de Dirac e Majorana a fim de estabelecer suas diferenças. Como os neutrinos possuem massa, seja um neutrino massivo com helicidade negativa, $\nu(\vec{p},-h)$. A invariância $C P T$ (conjugação de carga-paridade-reversão temporal ) requer que exista a imagem $C P T$ do $\nu(\vec{p},-h)$ que consiste de um antineutrino com helicidade positiva, $\bar{\nu}(\vec{p}, h)$

$$
(\underbrace{\nu(\vec{p},-h), \quad \bar{\nu}(\vec{p}, h)}_{C P T}) .
$$

Já que o $\nu$ possui massa, é possível encontrar um referencial que se mova mais rapidamente que $\nu$ de modo que, neste referencial, o $\nu$ esteja se movendo em sentido contrário mas não se veja inversão de seu spin. Assim, através de um boost de Lorentz teremos transformado o neutrino de helicidade negativa em um neutrino de helicidade positiva $\nu(-\vec{p}, h)$, seguido de uma rotação de $180^{\circ}$ obtemos um neutrino de momento e helicidade positivos $\nu(\vec{p}, h)$ :

$$
(\underbrace{\overbrace{\nu(\vec{p},-h), \quad \bar{\nu}(\vec{p}, h)}^{\text {boost+rotation } 180^{\circ}})(\cdots, \quad \nu(\vec{p}, h)}_{C P T}) .
$$

Aqui surge a seguinte questão: os dois estados de helicidade positiva são o mesmo estado ? Façamos duas suposições:

- Suposição 1: $\nu(\vec{p}, h)$ não é idêntico a $\bar{\nu}(\vec{p}, h)$.

Neste caso $\nu(\vec{p}, h)$ tem sua própria imagem $C P T$ que é $\bar{\nu}(\vec{p},-h)$. Este novo estado pode ser conectado com $\bar{\nu}(\vec{p}, h)$ por meio de uma transformação de Lorentz. Assim temos quatro estados com o mesmo momento. Este conjunto de estados constitui o neutrino de Dirac $\nu^{D}$.

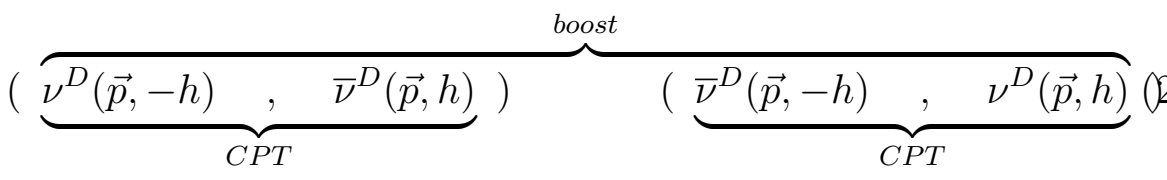


- Suposição 2: $\nu(\vec{p}, h)$ é idêntico a $\bar{\nu}(\vec{p}, h)$.

Agora ficamos simplesmente com apenas dois estados do mesmo momento

$$
(\overbrace{C P T}^{\overbrace{\nu(\vec{p},-h), \quad \nu(\vec{p}, h)}^{\text {boost }}}) .
$$

Este conjunto de dois estados é o que se chama de um neutrino de neutrino de Majorana $\nu^{M}$

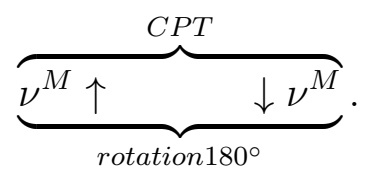

Um espinor de Dirac é definido por

$$
\psi=\left(\begin{array}{c}
\psi_{L} \\
\psi_{R}
\end{array}\right),
$$

onde $\psi_{L}$ e $\psi_{R}$ são espinores quirais, como um espinor quiral é a menor representação irredutível do grupo de Lorentz, se pode observar da definição do espinor de Dirac, que este não é uma representação irredutível do grupo de Lorentz.

Um espinor de Majorana $\psi^{M}$ é definido como

$$
\psi^{M}=\left(\begin{array}{c}
\psi_{L} \\
-i \tau^{2} \psi_{L}
\end{array}\right)
$$

e satisfaz a condição de

$$
\psi^{c}=\psi, \operatorname{com} \quad \psi^{c}=C \bar{\psi}^{T},
$$

como o espinor de Majorana ao ser construído por apenas um espinor quiral, este é uma representação irredutível do grupo de Lorentz. Agora vamos estudar a diferença entre os termos de massa para neutrinos de Dirac e Majorana. 


\subsubsection{Massa do Neutrino Tipo Dirac}

Um neutrino massivo de Dirac pode ser gerado com o mesmo mecanismo de Higgs que fornece massa para os léptons carregados e os quarks no MP. A extensão no MP que é necessária é a introdução de componentes de mão direita $\nu_{R}$ dos campos dos neutrinos $(\alpha: e, \mu, \tau)$. Tal modelo é chamado Extensão Mínima do Modelo Padrão. Uma importante diferença do neutrino de mão direita com os outros férmions elementares, é que eles são singletos do $S U(3)_{C} \times S U(2)_{L}$ e tem hipercarga $Y=0$. Os neutrinos de mão direita são chamados de neutrinos estéreis, porque sua única interação é a gravitacional. Por outro lado, os neutrinos de mão esquerda que participam na interação fraca são chamados de neutrinos ativos.

Na Extensão Mínima do MP com três neutrinos de mão direita, a lagrangiana Yukawa-Léptons-Higgs na equação (2.58) é estendida pela adição de um termo com a mesma estrutura como o segundo termo da equação (2.72) que gera massa para os quarks do tipo up:

$$
\mathcal{L}_{H L}=-\left[Y_{\alpha \beta}^{\prime l} \overline{L_{\alpha L}} \Phi l_{\beta R}^{\prime}+Y_{\alpha \beta}^{\prime \nu} \overline{L_{\alpha L}^{\prime}} \tilde{\Phi} l_{\beta R}^{\prime}\right]+\text { h.c. }
$$

Depois da quebra espontânea da simetria e as transformações

$$
V_{L}^{\nu \dagger} Y^{\prime \nu} V_{R}^{v}=Y^{\nu}, \quad \operatorname{com} \quad Y_{k, j}^{\nu}=y_{k}^{\nu} \delta_{k j} \quad(k, j=1,2,3)
$$

$\mathrm{e}$

$$
\nu_{k L}=\left(V_{L}^{\nu \dagger}\right)_{k \alpha} \nu_{\alpha L}^{\prime}, \quad \nu_{k R}=\left(V_{R}^{\nu \dagger}\right)_{k \alpha} \nu_{\alpha R}^{\prime}
$$

A lagrangiana Yukawa-Léptons-Higgs na equação (2.101) escreve-se como

$$
\mathcal{L}_{H L}=-\frac{v+h}{\sqrt{2}}\left[y_{\alpha}^{l} \overline{l_{\alpha L}} l_{\alpha R}+y_{k}^{\nu} \overline{\nu_{k L}} \nu_{k R}\right] .
$$

Com massa para os neutrinos dado por

$$
m_{k}=\frac{y_{k}^{\nu} v}{\sqrt{2}}
$$

Usando a transformações (2.62) e (2.103), que definem os campos para léptons e neutrinos massivos, a corrente carregada na equação (2.12) se pode escrever como

$$
j_{W, L}^{u}=2 \overline{l^{\prime}}{ }_{L} \gamma^{\mu} \nu^{\prime}{ }_{L}=2 \overline{l_{L}} V_{L}^{l \dagger} \gamma^{\mu} V_{L}^{\nu} \nu_{L}=2 \overline{\nu_{L}} V_{L}^{\nu+} V_{L}^{l} \gamma^{\mu} l_{L}
$$


Esta corrente depende do produto $U_{P M N S}=V_{L}^{l \dagger} V_{L}^{\nu}$ que é a chamada matriz de Pontecorvo Maki Nakagawa Sakata (PMNS), usando a definição dos neutrinos de sabor (2.67),

$$
\nu_{\alpha L}=V_{\alpha L}^{l+} \nu_{L}^{\prime}=\left[V_{L}^{\nu+} V_{L}^{l}\right]_{\alpha k} \nu_{k L}=\left(U_{P M N S}\right)_{\alpha k} \nu_{k L},
$$

pode-se observa que a matriz $U_{P M N S}$ relaciona os estados de sabor com os estados de massa para os neutrinos. Para o caso de neutrinos de Dirac a parametrização padrão para a matriz $U_{P M N S}$

$$
U_{P M N S}=\left(\begin{array}{ccc}
c_{12} c_{13} & s_{12} s_{13} & s_{13} e^{-i \delta} \\
-s_{12} c_{23}-c_{12} s_{23} s_{13} e^{i \delta} & c_{12} c_{23}-s_{12} s_{23} s_{13} e^{i \delta} & s_{23} c_{13} \\
s_{12} s_{23}-c_{12} c_{23} s_{13} e^{i \delta} & -c_{12} s_{23}-s_{12} c_{23} s_{13} e^{i \delta} & c_{23} c_{13}
\end{array}\right) .
$$

Onde $c_{i j} \equiv \cos \theta_{i j}$ e $s_{i j} \equiv \sin \theta_{i j}$. O ângulo $\theta_{i j}$ pode ser escolhido sem perdida de generalidade no primeiro quadrante, $\theta_{i j} \in[0, \pi / 2]$ e a fases $\delta \in[0,2 \pi]$.

\subsubsection{Massa do Neutrino Tipo Majorana}

Para entender uma teoria com neutrinos de Majorana, considera-se um só neutrino do tipo $\nu_{L}$. Uma massa de Majorana é gerada por um termo de massa da lagrangiana com só um campo quiral [9]. Como só se conhecem neutrinos de mão esquerda, só pode ser utilizados campos quirais de mão esquerda $\nu_{L}$. Então é possível escrever um termo de massa usando só $\nu_{L}$. Para responder isto considere-se primeiro o termo de massa de Dirac para os neutrinos equação (2.104)

$$
\mathcal{L}_{\text {massa }}^{D}=m \overline{\nu_{L}} \nu_{R}+\text { h.c. }
$$

O termo de massa na equação (2.109) é um escalar de Lorentz, como todos os termos da lagrangiana tem que ser, porque sob uma transformação de Lorentz $S$, os campos quirais $\nu_{L}$ e $\nu_{R}$ transformam-se como

$$
\begin{array}{cc}
\nu_{L}(x) \stackrel{S}{\rightarrow} S \nu_{L}(x), & \nu_{R}(x) \stackrel{S}{\rightarrow} S \nu_{R}(x), \\
\overline{\nu_{L}} \stackrel{S}{\rightarrow}{\overline{\nu^{\prime}}}_{L}(x) S^{-1}, & \overline{\nu_{R}} \stackrel{S}{\rightarrow}{\overline{\nu^{\prime}}}_{R}(x) S^{-1} .
\end{array}
$$

Para escrever um termo de massa de Majorana usando só $\nu_{L}$, tem-se que encontrar uma função de mão direita de $\nu_{L}$ que se transforme como $\nu_{R}$ sob 
a transformação de Lorentz $S$ e pode substituir $\nu_{R}$ na equação (2.110). Tal função de $\nu_{L}$ é campo de conjugação de carga

$$
\nu_{L}^{c}=C{\overline{\nu_{L}}}^{T} .
$$

Como $\nu_{L}^{c}$ é de mão direita, $\overline{\nu_{L}^{c}} \nu_{L}$, é não nulo. Portanto, sob a transformação de Lorentz $S$ o campo $\nu_{L}^{c}$ transforma-se como:

$$
\nu_{L}^{c}(x)=C\left(\overline{\nu_{L}}\right)^{T} \stackrel{S}{\rightarrow} C\left({\overline{\nu_{L}}}^{\dagger}\right)^{T}=C\left(S^{-1}\right)^{T}{\overline{\nu_{L}}}^{T}=C\left(S^{-1}\right)^{T} C^{-1} \nu_{L}^{c},
$$

usando $C\left(S^{-1}\right)^{T} C^{-}=S$, pode-se observar que $\nu_{L}^{c}$ transforma-se como $\nu_{L}$, enquanto $\overline{\nu_{L}^{c}}$ transforma-se como $\overline{\nu_{L}}$,

$$
\nu_{L}^{c}(x) \stackrel{S}{\rightarrow} S \nu_{L}^{c}(x), \quad \overline{\nu_{L}^{c}} \stackrel{S}{\rightarrow} \overline{\nu_{L}^{c}} S^{-1} .
$$

Assim, $\nu_{L}^{c}$ tem as propiedades certas para ser usado no lugar de $\nu_{R}$ na equação (2.109), deixando um termo de massa de Majorana invariante sob a transformação de Lorentz $S$ como:

$$
\mathcal{L}_{\text {massa }}^{M}=\frac{m}{2} \overline{\nu_{L}^{c}} \nu_{L}+\text { h.c }=\frac{m}{2} \nu_{L}^{T} C \nu_{L}+\text { h.c. }
$$

Na equação (2.105) pode-se observar que as massas para os neutrinos são proporcionais ao VEV do Higgs $v$, como as massas para os léptons e os quarks das equações (2.65) e (2.80). Não obstante, é bem conhecido que as massas dos neutrinos são muito menores que os léptons carregados e os quark. No mecanismo descrito para obter uma massa de Dirac para o neutrino, não existe explicação dos valores muito pequenos para os acoplamentos que são necessários para obter os valores pequenos para as massas dos neutrinos. De fato, o mecanismo de Higgs discutido neste capítulo deixa aberta completamente a questão dos valores dos acoplamentos de Yukawa com o Higgs de todas as partículas. Por tanto, a origem das massas para os quarks e léptons é um mistério no marco do MP. Assim, a Extensão Mínima do Modelo Padrão não pode explicar os valores muito pequenos das massas dos neutrinos.

Por outro lado, é um fato que o MP com a simetria de gauge $S U(3)_{C} \times$ $S U(2)_{L} \times U(1)_{Y}$ apresenta uma acidental simetria global [12]:

$$
G_{S M}^{\text {global }}=U_{B} \times U_{L_{e}} \times U_{L_{\mu}} \times U_{L_{\tau}}
$$

onde $U_{B}$ é a simetria bariônica, e $U_{L_{e}, L_{\mu}, L_{\tau}}$ são as três simetrias de sabor leptônico, com número leptônico total dado por $L=L_{e}+L_{\mu}+L_{\tau}$. 
Em princípio a massa dos neutrinos poderia vir de correções de loops. No MP, portanto, isso não acontece porque o único termo possível de massa para os neutrinos que pode ser construído com campos do MP é o bilinear $\overline{\nu_{L}} \nu_{L}^{C}$ equação (2.114), que viola a simetria global leptônica por duas unidades. Como foi mencionado antes o número leptônico total é uma simetria global do MP e portanto termos que violem o número leptônico não podem ser induzidos por correções de loops. Além disso, o subgrupo $U_{B-L}$ de $G_{S M}^{\text {global }}$ é não anômalo [13], e portanto termos que violem $B-L$ não podem ser induzidos por correções não perturbativas. Assim, um termo de massa de Majorana não pode ser construído no contexto do MP. Disso segue que o MP prediz que os neutrinos são precisamente não massivos. Para adicionar massa aos neutrinos o MP precisa ser estendido.

Entre outros problemas, este mistério nos leva a crer que o MP deve ser considerado como uma teoria efetiva obtida no limite de uma teoria mais completa, em que as massas dos quarks e léptons podem ser derivadas dos primeiros princípios. Assim, os valores das massas dos quarks e léptons representam um identificador útil que pode nos permitir compreender a física além do MP. De fato, a comparação dos valores previstos com os valores observados para os espectros nas massas dos quarks e léptons fornecem fortes restrições que nos permitem selecionar entre novas teorias. Neste contexto, os valores muito pequenos das massas dos neutrinos é uma restrição muito forte que as novas teorias devem satisfazer a baixas energias. No próximo capítulo se estudará os modelos seesaw que são extensões do MP onde é possível gerar o termo de massa para o neutrino. 


\section{Capítulo 3}

\section{Modelos Seesaw}

Neste capítulo vamos estudar separadamente três extensões do MP no contexto de uma teoria efetiva a baixa energia. Estas extensões resultam na adição de singletos de férmions ou tripletos escalares ou tripletos de férmions para o conteúdo de campos do MP.

\subsection{Seesaw Tipo I}

Considere-se a extensão mínima do Modelo Padrão com três gerações onde $n$ neutrinos de mão direita são adicionados. A lagrangiana mais geral renormalizável e invariante de gauge [18] é dada por

$$
\mathcal{L}=\mathcal{L}_{M P}+\mathcal{L}_{N_{R}}
$$

onde $\mathcal{L}_{M P}$ é a lagrangiana do MP, e

$\mathcal{L}_{N_{R}}=i \overline{N_{R}} \not \partial N_{R}-\left(\overline{L_{L}} \widetilde{\phi} Y_{\nu}^{\dagger} N_{R}+\overline{N_{R}} Y_{\nu} \widetilde{\phi}^{\dagger} L_{L}\right)-\frac{1}{2}\left(\overline{N_{R}^{c}} M_{R} N_{R}+\overline{N_{R}} M_{R}^{*} N_{R}^{c}\right)$.

$\mathcal{L}_{N_{R}}$ contém os termos de energia cinética de massa de Majorana para os neutrinos de mão direita, e interações de Yukawa entre os neutrinos de mão direita, os dubletos de léptons de mão esquerda e o dubleto de Higgs. Como os $N_{R}$ são singletos de $S U(3)_{C} \times S U(2)_{L}$ e tem $Y=0$, a derivada covariante reduz-se a $\partial_{\mu}$ no termo da energia cinética. A matriz de massa $M_{R}$ é uma matriz $n \times n$ simétrica complexa. A matriz de massa de Majorana tem, em 
geral, $n$ autovalores complexos $M_{R i}=e^{i \theta_{i} / 2}\left|M_{i}\right| \equiv \eta_{i}\left|M_{i}\right|$ que dependem das fases de Majorana $\theta_{i}$ dos neutrinos de Majorana pesados. Pode-se trabalhar na base em que $M$ é real e diagonal. Neste caso, os autoestados de massa dos neutrinos de Majorana $N_{i}=N_{i}^{c}$ são dados por [12]

$$
N_{i} \equiv e^{i \theta_{i} / 2} N_{R i}+e^{-i \theta_{i} / 2} N_{R i}^{c}=\sqrt{\eta_{i}} N_{R i}+\sqrt{\eta_{i}^{*}} N_{R i}^{c} .
$$

Usando

$$
\begin{aligned}
\overline{L_{L}^{c}} \widetilde{\phi}^{*} Y_{\nu}^{T} \sqrt{\eta} N & =\left(L_{L}^{T} C \widetilde{\phi}^{*}\right)_{1 \alpha}\left(Y_{\nu}^{T} \sqrt{\eta}\right)_{\alpha \beta} N_{\beta 1}=\left(\widetilde{\phi}^{\dagger} L_{L}\right)_{\alpha 1}\left(\sqrt{\eta} Y_{\nu}\right)_{\beta \alpha} C N_{\beta 1} \\
& =\left(\widetilde{\phi}^{\dagger} L_{L}\right)_{\alpha 1}\left(\sqrt{\eta} Y_{\nu}\right)_{\beta \alpha}\left(-\overline{N_{\beta 1}^{T}}\right)=\overline{N_{1 \beta}}\left(\widetilde{\phi}^{\dagger} L_{L}\right)_{\alpha 1}\left(\sqrt{\eta} Y_{\nu}\right)_{\beta \alpha} \\
& =\bar{N} \sqrt{\eta} Y_{\nu} \widetilde{\phi}^{\dagger} L_{L},
\end{aligned}
$$

a lagrangiana em (3.2) pode ser escrita como

$$
\begin{aligned}
\mathcal{L}_{N} & =\frac{1}{2} \overline{N_{i}}\left(i \not \partial-M_{i}\right) N_{i} \\
& -\frac{1}{2}\left[\overline{L_{L}} \widetilde{\phi} Y_{\nu}^{\dagger} \sqrt{\eta^{*}}+\overline{L_{L}^{c}} \widetilde{\phi}^{*} Y_{\nu}^{T} \sqrt{\eta}\right]_{i} N_{i}+\frac{1}{2} \overline{N_{i}}\left[\sqrt{\eta^{*}} Y_{\nu}^{*} \widetilde{\phi}^{T} L_{L}^{c}+\sqrt{\eta} Y_{\nu} \widetilde{\phi}^{\dagger} L_{L}\right]_{i},
\end{aligned}
$$

onde $\eta$ é a matriz $n \times n$ diagonal com elementos $\eta_{i}$.

Uma lagrangiana efetiva que é valida a energias menores que $M$, onde $M$ é a massa dos neutrinos pesados, pode ser construída por integração dos neutrinos pesados de Majorana $N_{i}$. A lagrangiana efetiva tem uma expansão em série de potência de $1 / M$ da forma

$$
\mathcal{L}_{\text {eff }}=\mathcal{L}_{M P}+\frac{1}{M} \mathcal{L}^{d=5}+\frac{1}{M^{2}} \mathcal{L}^{d=6}+\ldots \equiv \mathcal{L}_{M P}+\delta \mathcal{L}^{d=5}+\delta \mathcal{L}^{d=6}+\ldots
$$

para explicar os efeitos físicos dos neutrinos pesados de Majorana para energias $\leq M$. A lagrangiana efetiva é definida através da ação efetiva [19]

$$
e^{i S_{\text {eff }}}=\exp \left[i \int d^{4} x \mathcal{L}_{e f f}\right]=e^{i S_{M P}} \int \mathcal{D} \bar{N} \mathcal{D} N e^{i \int d^{4} x \mathcal{L}_{N}} .
$$

A lagrangiana efetiva pode ser encontrada por integração funcional de todos os campos pesados de Majorana na equação (3.7). Escrevendo $S$ como a ação associada a lagrangiana em (3.5), as equações clássicas de movimento para os campos $N$ com solução $N_{0}$ são obtidas de

$$
\left.\frac{\delta S}{\delta N_{i}(x)}\right|_{N_{0 i}}=0,\left.\quad \frac{\delta S}{\delta \bar{N}_{i}(x)}\right|_{N_{0 i}}=0
$$


que fornecem

$$
\begin{aligned}
\bar{N}_{0 i}\left(-i \overleftarrow{\not \partial}-M_{i}\right)-\left[\overline{L_{L}} \widetilde{\phi} Y_{\nu}^{\dagger} \sqrt{\eta *}+\overline{L_{L}^{c}} \widetilde{\phi}^{*} Y_{\nu}^{T} \sqrt{\eta}\right] & =0 \\
\left(i \overrightarrow{\not \partial}-M_{i}\right) N_{0 i}-\left[\sqrt{\eta *} Y_{\nu}^{*} \widetilde{\phi}^{T} L_{L}^{c}+\sqrt{\eta} Y_{\nu} \widetilde{\phi}^{\dagger} L_{L}\right] & =0 .
\end{aligned}
$$

Tendo encontrado as equações para os campos clássicos $N_{0}$, seguindo o procedimento dado na referência [19], o expoente $i \int d^{4} x \mathcal{L}_{N}$ em (3.7) tem que ser expandido entorno do campo $N_{0}$. Substituindo $N=N_{0}+\widehat{N}$ neste expoente, (3.7) é escrita como

$e^{i S_{e f f}}=e^{i S_{M P}} \int \mathcal{D} \widehat{\mathcal{N}} \mathcal{D} \widehat{N} e^{\left[i \int d^{4} x \mathcal{L}_{N_{0}}+i \int \overline{\widehat{N}}\left(\left.\frac{\delta L}{\delta N(x)}\right|_{N_{0}}\right)+i \int \widehat{N}\left(\left.\frac{\delta L}{\delta \bar{N}(x)}\right|_{N_{0}}\right)+\frac{i}{2} \int \overline{\overline{N(x)}}\left[\left.\frac{\delta^{2} L}{\delta N(x) \bar{N}(y) \delta}\right|_{N_{0}}\right] \widehat{N}(y)\right]}$.

Nesta equação os termos lineares em $\widehat{N}$ e $\widehat{N}$ são nulos, produto das equações clássicas (3.8), assim (3.10) é escrita como

$$
e^{i S_{e f f}}=e^{i S_{M P}+i \int d^{4} x \mathcal{L}_{N_{0}}}\left(\int \mathcal{D} \overline{\widehat{N}} \mathcal{D} \widehat{N} e^{\frac{i}{2} \int \overline{\widehat{N(x)}}\left[\left.\frac{\delta^{2} L}{\delta N(x) \bar{N}(x) \delta}\right|_{N_{0}}\right] \widehat{N}(y)}\right) .
$$

Os termos entre parênteses em (3.11) são integrais gaussianas, como é bem conhecido estas integrais podem ser calculas levando ao aparecimento de determinantes funcionais. Como (3.5) tem termos até segunda ordem em $N$, estas integrais gaussianas são constantes e não contribuem para $S_{\text {eff }}$, assim a ação efetiva para o modelo é dado por

$$
S_{e f f}=S_{M P}+S_{N}\left[N_{0}\right],
$$

onde

$$
S_{N}\left[N_{0}\right]=\int d^{4} x \mathcal{L}_{N_{0}} .
$$

Usando (3.9) em (3.5) obtém-se

$$
\begin{aligned}
\mathcal{L}_{N_{0}}= & -\frac{1}{2}\left[\overline{L_{L}} \widetilde{\phi} Y_{\nu}^{\dagger} \sqrt{\eta *}+\overline{L_{L}^{c}} \widetilde{\phi}^{*} Y_{\nu}^{T} \sqrt{\eta}\right] \\
& \left(\frac{\delta_{i j}}{i \overrightarrow{\not \partial}-M_{i}}\right)\left[\sqrt{\eta *} Y_{\nu}^{*} \widetilde{\phi}^{T} L_{L}^{c}+\sqrt{\eta} Y_{\nu} \widetilde{\phi}^{\dagger} L_{L}\right] .
\end{aligned}
$$


Da lagrangiana $\mathcal{L}_{N_{0}}$, todas as contribuições para a lagrangiana efetiva podem ser obtidas expandindo o propagador do neutrino pesado em séries de potência de $1 / M$ :

$$
\frac{1}{i \overrightarrow{\not \partial}-M_{i}}=-\frac{1}{M}-\frac{i \overrightarrow{\not \partial}}{M^{2}}+\ldots
$$

\subsubsection{Operador de dimensão $d=5$}

Das equações (3.14) e (3.15) o operador de dimensão 5 da lagrangiana efetiva para o modelo seesaw tipo I é dado por

$$
\begin{aligned}
\delta \mathcal{L}^{d=5} & =\frac{1}{2}\left[\overline{L_{L}} \widetilde{\phi} Y_{\nu}^{\dagger} \sqrt{\eta *}+\overline{L_{L}^{c}} \widetilde{\phi}^{*} Y_{\nu}^{T} \sqrt{\eta}\right]\left(\frac{1}{M}\right)\left[\sqrt{\eta^{*}} Y_{\nu}^{*} \widetilde{\phi}^{T} L_{L}^{c}+\sqrt{\eta} Y_{\nu} \widetilde{\phi}^{\dagger} L_{L}\right] \\
& =\frac{1}{2}\left[\overline{L_{L}} \widetilde{\phi} Y_{\nu}^{\dagger} \frac{\eta^{*}}{M} Y_{\nu}^{*} \widetilde{\phi}^{T} L_{L}^{c}+\overline{L_{L}^{c}} \widetilde{\phi}^{*} Y_{\nu}^{T} \frac{\eta}{M} Y_{\nu} \widetilde{\phi}^{\dagger} L_{L}\right] \\
& =\frac{1}{2} c_{\alpha \beta}^{d=5}\left(\overline{L_{\alpha L}^{c}} \widetilde{\phi}^{*}\right)\left(\widetilde{\phi}^{\dagger} L_{\beta L}\right)+\frac{1}{2}\left(c_{\alpha \beta}^{d=5}\right)^{*}\left(\overline{L_{L}} \widetilde{\phi}\right)\left(\widetilde{\phi}^{T} L_{L}^{c}\right)
\end{aligned}
$$

onde

$$
c_{\alpha \beta}^{d=5}=\left(Y_{\nu}^{T} \frac{\eta}{M} Y_{\nu}\right)_{\alpha \beta}=\left(Y_{\nu}^{T} \frac{1}{M_{R}} Y_{\nu}\right)_{\alpha \beta}
$$

Usando a identidade $\tau_{\alpha \beta}^{\mu} \tau_{\mu \theta \sigma}=-2 \tau_{\alpha \theta}^{2} \tau_{\beta \sigma}^{2}, \widetilde{\phi}^{\dagger} \phi=0$ e a definição $\widetilde{L} \equiv i \tau_{2} L^{c}$ a equação (3.16) pode ser expressa como ${ }^{1}$

$$
\delta \mathcal{L}^{d=5}=-\frac{1}{4} c_{\alpha \beta}^{d=5}\left(\overline{\widetilde{L}_{\alpha L}} \vec{\tau} L_{\beta L}\right)\left(\widetilde{\phi}^{\dagger} \vec{\tau} \phi\right)+h . c,
$$

que é o conhecido operador de dimensão $d=5$ [8] que gera massas de Majorana para os neutrinos $\nu_{L}$ quando o dubleto de Higgs adquire um valor esperado de vácuo não nulo $v / \sqrt{2}=174 \mathrm{GeV}$. A matriz de massa para os neutrinos $\nu_{L}$ é dada por

$$
m_{\alpha \beta}=-\frac{v^{2}}{2} c_{\alpha \beta}^{d=5}=-\frac{v^{2}}{2}\left(Y_{\nu}^{T} \frac{1}{M_{R}} Y_{\nu}\right)_{\alpha \beta}
$$

\footnotetext{
${ }^{1}$ A expressão em (3.18) é apropiada para poder identificar os coeficientes $c^{d=5}$ nos modelos seesaw tipo II e tipo III.
} 


\subsubsection{Operador de dimensão $d=6$}

Das equações (3.14) e (3.15) o operador de dimensão $d=6$ da lagrangiana efetiva para o modelo seesaw tipo I é dado por

$\delta \mathcal{L}^{d=6}=\frac{1}{2}\left[\overline{L_{L}} \widetilde{\phi} Y_{\nu}^{\dagger} \sqrt{n *}+\overline{L_{L}^{c}} \widetilde{\phi}^{*} Y_{\nu}^{T} \sqrt{n}\right]\left(\frac{i \vec{\not}}{M^{2}}\right)\left[\sqrt{n *} Y_{\nu}^{*} \widetilde{\phi}^{T} L_{L}^{c}+\sqrt{n} Y_{\nu} \widetilde{\phi}^{\dagger} L_{L}\right]$.

Usando

$$
\overline{L_{\alpha L}^{c}} A_{\alpha \beta} \not \partial L_{\beta L}^{c}=L_{\alpha L} A_{\alpha \beta}^{T} \not \partial L_{\beta L},
$$

onde $A_{\alpha \beta}$ é uma matriz $3 \times 3$ complexa a equação (3.20) pode ser escrita como

$$
\delta \mathcal{L}^{d=6}=c_{\alpha \beta}^{d=6}\left(\overline{L_{\alpha L}} \widetilde{\phi}\right) i \overrightarrow{\not \partial}\left(\widetilde{\phi}^{\dagger} L_{\beta L}\right)
$$

onde

$$
c_{\alpha \beta}^{d=6}=\left(Y_{\nu}^{\dagger} \frac{1}{M^{2}} Y_{\nu}\right)_{\alpha \beta}=\left(Y_{\nu}^{\dagger} \frac{1}{M_{R}^{\dagger} M_{R}} Y_{\nu}\right)_{\alpha \beta},
$$

que é da mesma ordem em acoplamento de Yukawa que $c_{\alpha \beta}^{d=5}$, equação (3.17), enquanto dependente do quadrado do inverso de $M_{R}$. Quando o dubleto de Higgs adquire um valor esperado de vácuo não nulo $v / \sqrt{2}$, o operador de dimensão $d=6$ leva a correções para o termo cinético dos neutrinos. A lagrangiana para os léptons para a teoria efetiva, incluindo só operadores de dimensão $d \leq 6$ e não considerando o acoplamento com o Higgs, é dada por

$$
\begin{aligned}
\mathcal{L}_{\text {léptons }}^{d \leq 6} & =i \overline{\nu_{\alpha L}} \not \partial\left(\delta_{\alpha \beta}+\epsilon_{\alpha \beta}\right) \nu_{\beta L}-i \overline{\bar{l}_{\alpha L}} \not \partial l_{\alpha L}-\frac{1}{2}\left(\overline{\nu_{\alpha L}^{c}} m_{\nu \alpha \beta} \nu_{\alpha L}+h . c\right) \\
& -\overline{l_{\alpha L}} m_{l \alpha \beta} \nu_{\beta L}+\mathcal{L}^{(C C)}+\mathcal{L}^{(N C)}+\mathcal{L}^{\gamma}
\end{aligned}
$$

onde

$$
\epsilon_{\alpha \beta}=\frac{v^{2}}{2} c_{\alpha \beta}^{d=6}
$$

é a contribuição do coeficiente do operador $d=6$ para a energia cinética dos neutrinos de mão esquerda que, como se pode observar, não é diagonal no 
espaço de sabor. $m_{l}$ é a matriz de massa para os léptons carregados e $\mathcal{L}^{(C C)}$ e $\mathcal{L}^{(N C)}$ são as lagrangianas de interação carregada e neutra, respectivamente.

Como as matrizes de massa $m_{\nu}$ e $m_{l}$ não são diagonais e a fim de obter-se um termo da energia cinética, para o neutrino, canonicamente normalizada, pode-se rodar a base para a qual as matrizes de massa são diagonais e o termo cinético é canonicamente normalizado, tal base e definida como

$$
\nu_{i}=V_{i \alpha}^{e f f} \nu_{\alpha L}+V_{i \alpha}^{e f f *} \nu_{\alpha L}^{c}, \text { com } \quad V^{e f f}=U^{\dagger}\left(1+\frac{\epsilon}{2}\right)
$$

onde $U$ é a matriz que diagonaliza a matriz de massa do neutrino. Em termos dos autoestados de massa, e considerando termos até $\mathcal{O}\left(1 / M^{2}\right)$ a lagrangiana para os léptons é dada por

$$
\mathcal{L}_{\text {léptons }}^{d \leq 6}=\frac{1}{2} i \overline{\nu_{i}}\left(\not \partial-m_{\nu i}^{\text {diag }}\right) \nu_{i L}-\overline{l_{\alpha L}}\left(i \not \partial-m_{l \alpha}^{\text {diag }}\right) l_{\alpha L}+\mathcal{L}^{(C C)}+\mathcal{L}^{(N C)}
$$

onde, nesta base de massa, as lagrangianas $\mathcal{L}^{(C C)}$ e $\mathcal{L}^{(N C)}$, se escrevem como

$$
\begin{aligned}
\mathcal{L}_{I, L}^{(C C)} & =\frac{g}{\sqrt{2}} \bar{l}_{\alpha L} \gamma^{\mu} N_{\alpha i} \nu_{i L} W_{\mu}^{-}+h . c \\
\mathcal{L}_{I, L}^{(N C)} & =\frac{g}{2 \cos \theta_{W}}\left[\bar{\nu}_{i L} \not Z\left(N^{\dagger} N\right)_{i j} \nu_{j_{L}}-\bar{l}_{\alpha L} \not Z l_{\alpha L}+2 \sin ^{2} \theta_{W} \bar{l}_{\alpha} \not Z l_{\alpha}\right] \\
& -e \bar{l}_{\alpha} A l_{\alpha}
\end{aligned}
$$

com

$$
N \equiv \Omega\left(1-\frac{\epsilon}{2}\right) U
$$

onde $\Omega \equiv \operatorname{diag}\left(e^{i w_{1}}, e^{i w_{2}}, e^{i w_{3}}\right)$ reabsorve as três fases não físicas na definição de léptons carregados, como é usual. Em termos da matriz $N$, as correntes neutras e carregadas definidas em (3.28) são

$$
\begin{gathered}
j_{W, L}^{u}=2 \bar{l}_{\alpha L} \gamma^{u} N_{\alpha i} \nu_{i L} \\
J_{Z, L}^{u}=\bar{\nu}_{i} \gamma^{u}\left(N^{\dagger} N\right)_{i j} \nu_{j L},
\end{gathered}
$$

onde

$$
\left(N^{\dagger} N\right)_{i j}=\left(U^{\dagger}(1-\epsilon) U\right)_{i j} \neq \delta_{i j}
$$


aparece na corrente neutra para os neutrinos desde que $N$ é não unitária. Entretanto a corrente neutra para os léptons carregados não é modificada. A constante de Fermi $G_{F}$, não pode ser mais identificada pela combinação, a nível árvore no $\mathrm{MP}, G_{F}^{M P}=\sqrt{2} g^{2} /\left(8 M_{W}^{2}\right)$, devido à não unitariedade de $N$. Usando a equação (3.30), a constante de Fermi extraída do decaimento $\mu \rightarrow e \bar{\nu}_{e} \nu_{\mu}$ é relacionada com $G_{F}^{M P}$ por

$$
G_{F}=G_{F}^{M P} \sqrt{\left(N N^{\dagger}\right)_{e e}\left(N N^{\dagger}\right)_{\mu \mu}} .
$$

O resto de parâmetros da lagrangiana coincide com o tratamento padrão. É importante resaltar que os supostos desvios da unitariedade da matriz de mistura leptônica podem ser relacionados com o coeficiente de operador $d=6$, já que de (3.29) se obtém

$$
\left(N N^{\dagger}\right)_{\alpha \beta}=\left[\Omega(1+\epsilon) \Omega^{\dagger}\right]_{\alpha \beta},
$$

portanto

$$
\left|N N^{\dagger}-1\right|_{\alpha \beta}=|\epsilon|_{\alpha \beta}=\frac{v^{2}}{2}\left|c^{d=6}\right|_{\alpha \beta}=\frac{v^{2}}{2}\left(Y_{\nu}^{\dagger} \frac{1}{M_{R}^{\dagger} M_{R}} Y_{\nu}\right)_{\alpha \beta} .
$$

\subsection{Seesaw Tipo II}

Considere-se a extensão do MP por adição de um tripleto escalar de $S U(2)_{L}$,

$$
\vec{\Delta}=\left(\Delta_{1}, \Delta_{2}, \Delta_{3}\right)
$$

com $Y=1$. A lagrangiana para o modelo seesaw tipo II [33] é

$$
\begin{aligned}
\mathcal{L}_{\Delta}= & \left(D_{\mu} \vec{\Delta}\right)^{\dagger}\left(D^{\mu} \vec{\Delta}\right)+\left[\overline{L_{L}} Y_{\Delta}^{\dagger}(\vec{\tau} \cdot \vec{\Delta})^{\dagger} \widetilde{L}_{L}+u_{\Delta} \widetilde{\phi}^{\dagger}(\vec{\tau} \cdot \vec{\Delta})^{\dagger} \phi+h . c\right] \\
& +\vec{\Delta}^{\dagger} M_{\Delta}^{2} \vec{\Delta}+\lambda_{3}\left(\phi^{\dagger} \phi\right)(\vec{\Delta} \vec{\Delta})+\lambda_{5}\left(\vec{\Delta}^{\dagger} T^{i} \vec{\Delta}\right) \phi^{\dagger} \tau^{i} \phi \\
& +\frac{1}{2} \lambda_{2}\left(\vec{\Delta}^{\dagger} \vec{\Delta}\right)^{2}+\frac{\lambda_{4}}{2}\left(\vec{\Delta}^{\dagger} T^{i} \vec{\Delta}\right)^{2},
\end{aligned}
$$

onde a soma sobre os índices $i$ de $S U(2)_{L}$ é assumida. Aqui supomos que a matriz $M_{\Delta}$ é real e diagonal e a derivada covariante em (3.37) é dada por

$$
D_{\mu} \equiv \partial_{\mu}-i g \vec{T} \cdot \vec{W}_{\mu}-i g^{\prime} B_{\mu} \frac{Y}{2}
$$


com $\vec{T}$ denotando a representação tridimensional dos geradores de $S U(2)_{L}$ :

$$
T_{1}=\left(\begin{array}{ccc}
0 & 0 & 0 \\
0 & 0 & -i \\
0 & -i & 0
\end{array}\right), \quad T_{2}=\left(\begin{array}{ccc}
0 & 0 & i \\
0 & 0 & 0 \\
-i & 0 & 0
\end{array}\right), \quad T_{3}=\left(\begin{array}{ccc}
0 & -i & 0 \\
i & 0 & 0 \\
0 & 0 & 0
\end{array}\right)(3 .
$$

Considerando-se que os escalares do tripleto são pesados, $M_{\Delta}>>v$ é suficiente resolver o problema em quarta ordem em $\vec{\Delta}$ para resolver a equação de movimento para $\vec{\Delta}$ e encontrar os termos dominantes da lagrangiana efetiva do modelo. Isto é, a lagrangiana não perturbada do modelo em segundo ordem sobre $\vec{\Delta}$ é

$$
\begin{aligned}
\mathcal{L}_{\Delta}^{0}= & \left(D_{u} \vec{\Delta}\right)^{\dagger}\left(D^{u} \vec{\Delta}\right)+\left[\overline{L_{L}} Y_{\Delta}^{\dagger}(\vec{\tau} \cdot \vec{\Delta})^{\dagger} \widetilde{L}_{L}+u_{\Delta} \widetilde{\phi}^{\dagger}(\vec{\tau} \cdot \vec{\Delta})^{\dagger} \phi+h . c\right] \\
& +\vec{\Delta}^{\dagger} M_{\Delta}^{2} \vec{\Delta}+\lambda_{3}\left(\phi^{\dagger} \phi\right)(\vec{\Delta} \vec{\Delta})+\lambda_{5}\left(\vec{\Delta}^{\dagger} T^{i} \vec{\Delta}\right) \phi^{\dagger} \tau^{i} \phi
\end{aligned}
$$

$\mathrm{e}$

$$
\frac{1}{2} \lambda_{2}\left(\vec{\Delta}^{\dagger} \vec{\Delta}\right)^{2}+\frac{\lambda_{4}}{2}\left(\vec{\Delta}^{\dagger} T^{i} \vec{\Delta}\right)^{2}
$$

como as perturbações. Para a lagrangiana não perturbada $\mathcal{L}_{\Delta}^{0}$ denotando a equação clássica de movimento para o campo $\Delta$ com solução $\Delta_{0}$,

$$
\left.\frac{\delta S_{\Delta}^{0}}{\delta \Delta_{i}(x)}\right|_{\Delta_{0 i}}=0
$$

com $S_{\Delta}^{0}$ como a ação da lagrangiana $\mathcal{L}_{\Delta}^{0}$, o campo clássico $\Delta_{0}^{\alpha}$ é dado por

$$
\Delta_{0}^{\alpha}=\left[D^{2}+M_{\Delta}^{2}+\lambda_{3}\left(\phi^{\dagger} \phi\right)+\lambda_{5} T^{i}\left(\phi^{\dagger} \tau^{i} \phi\right)\right]_{\alpha \beta}^{-1}\left[\overline{L_{L}} Y_{\Delta}^{\dagger} \tau^{\beta} \widetilde{L}_{L}+u_{\Delta} \widetilde{\phi}^{\dagger} \tau^{\beta} \phi\right] .
$$

Como no modelo seesaw tipo I, a ação $S^{0}$ tem termos até segunda ordem em $\vec{\Delta}$. Assim, a ação efetiva construída da lagrangiana não perturbada $\mathcal{L}_{\Delta}^{0}$ é dada por

$$
S_{e f f}=S_{M P}+S_{\Delta}^{0}\left[\Delta_{0}\right]
$$

onde

$$
S_{\Delta}^{0}\left[\Delta_{0}\right]=\int d^{4} x \mathcal{L}_{N_{0}}=\int d^{4} x\left[\overline{\widetilde{L}_{L}} Y_{\Delta}\left(\vec{\tau} \cdot \vec{\Delta}_{0}\right) L_{L}+u_{\Delta}^{*} \phi^{\dagger}\left(\vec{\tau} \cdot \overrightarrow{\Delta_{0}}\right) \widetilde{\phi}\right],
$$

$\operatorname{com} \overrightarrow{\Delta_{0}}$ definida na equação (3.43). 


\subsubsection{Operadores de dimensão $d=4$ e $d=5$}

Usando

$$
\frac{1}{y+M_{\Delta}^{2}}=\frac{1}{M_{\Delta}^{2}}-\frac{y}{M_{\Delta}^{2}}+\ldots, \operatorname{com}\left(y=D^{2}+\lambda_{3}\left(\phi^{\dagger} \phi\right)+\lambda_{5} T^{i}\left(\phi^{\dagger} \tau^{i} \phi\right)\right),
$$

na equação (3.43) pode-se expandir a lagrangiana efetiva (3.45) em potências inversas de $M_{\Delta}$. O produto disso é um operador de dimensão $d=4^{2}$ :

$$
\frac{\left|u_{\Delta}\right|^{2}}{M_{\Delta}^{2}}\left(\phi^{\dagger} \vec{\tau} \widetilde{\phi}\right)\left(\widetilde{\phi}^{\dagger} \vec{\tau} \phi\right)=2 \frac{\left|u_{\Delta}\right|^{2}}{M_{\Delta}^{2}}\left(\phi^{\dagger} \phi\right)^{2} .
$$

Também se obtém um operador de dimensão $d=5$, dado por

$$
\delta \mathcal{L}^{d=5}=\left(Y_{\Delta} \frac{u_{\Delta}}{M_{\Delta}^{2}}-\frac{2 \lambda_{3}\left(\phi^{\dagger} \phi\right)}{M_{\Delta}^{4}}\right)_{\alpha \beta}\left(\overline{\widetilde{L}}_{\alpha L} \vec{\tau} L_{\beta L}\right)\left(\widetilde{\phi}^{\dagger} \vec{\tau} \phi\right)+\text { h.c. }
$$

Considerando só o termo de $\mathcal{O}=1 / M_{\Delta}^{2}$, e comparando com $\delta \mathcal{L}^{d=5}$ dado na equação (3.18), o coeficiente do operador de $d=5$ é dado pela matriz

$$
c_{\alpha \beta}^{d=5}=4\left(Y_{\Delta} \frac{u_{\Delta}}{M_{\Delta}^{2}}\right)_{\alpha \beta},
$$

e analogamente como o modelo seesaw tipo I, o operador de dimensão $d=5$ gera massa de Majorana para os neutrinos, com a matriz de massa da forma

$$
m_{\alpha \beta}=-\frac{v^{2}}{2} c_{\alpha \beta}^{d=5}=-2 v^{2}\left(Y_{\Delta} \frac{u_{\Delta}}{M_{\Delta}^{2}}\right)_{\alpha \beta} .
$$

Pode-se observar que a massa do neutrino depende de $Y_{\Delta}$ e $u_{\Delta}$. Isto é esperado da lagrangiana (3.37), onde a quebra do número leptônico é produto da presença simultânea de $Y_{\Delta}$ e $u_{\Delta} \cdot{ }^{3}$

\footnotetext{
${ }^{2} \mathrm{Na}$ equação se usou a propriedade $\tau_{\alpha \beta}^{\mu} \tau_{\mu \theta \sigma}=2 \xi_{\alpha \theta} \xi_{\beta \sigma}$.

${ }^{3} \mathrm{Na}$ completa teoria seesaw tipo II, as massas dos neutrinos são obtidas quando a componente neutra $\Delta^{0}=\left(\Delta^{1}+i \Delta^{2}\right) / \sqrt{2}$ adquire um VEV $<\Delta^{0}>\equiv \nu / \sqrt{2}=u_{\Delta} v^{2} /\left(\sqrt{2} M_{\Delta}^{2}\right)$, [34].
} 


\subsubsection{Operador de dimensão $d=6$}

Substituindo as equações (3.43) e (3.46) em (3.45), o operador de dimensão $d=6$ da lagrangiana efetiva para o modelo seesaw tipo II é dado por

$$
\delta \mathcal{L}^{d=6}=\delta \mathcal{L}_{4 F}^{d=6}+\delta \mathcal{L}_{4 \phi}^{d=6}+\delta \mathcal{L}_{\phi D}^{d=6}
$$

onde

$$
\begin{gathered}
\delta \mathcal{L}_{4 F}^{d=6}=\left(\frac{1}{M_{\Delta}^{2}}-\frac{2 \lambda_{3}\left(\phi^{\dagger} \phi\right)}{M_{\Delta}^{4}}\right)\left(\overline{\widetilde{L}}_{L} Y_{\Delta} \vec{\tau} L_{L}\right)\left(\bar{L}_{L} Y_{\Delta}^{\dagger} \vec{\tau} \widetilde{L}\right) \\
\delta \mathcal{L}_{4 \phi}^{d=6}=-2\left(\lambda_{3}+\lambda_{5}\right) \frac{\left|u_{\Delta}\right|^{2}}{M_{\Delta}^{4}}\left(\phi^{\dagger} \phi\right)^{3} \\
\delta \mathcal{L}_{\phi D}^{d=6}=-\frac{\left|u_{\Delta}\right|^{2}}{M_{\Delta}^{4}}\left(\phi^{\dagger} \vec{\tau} \widetilde{\phi}\right)\left(D_{\mu}^{2}\right)\left(\widetilde{\phi}^{\dagger} \vec{\tau} \phi\right)
\end{gathered}
$$

com a derivada covariante definida em (3.38).

A lagrangiana $\delta \mathcal{L}_{4 F}^{d=6}$ pode ser escrita em uma forma mais familiar, usando $\left(\tau_{i}\right)_{a b}\left(\tau_{i}\right)_{c d}=2\left(\delta_{b c} \delta_{a d}-\frac{1}{2} \delta_{a b} \delta_{c d}\right)$ e a transformação de Fierz fornecida no apêndice da referência [20],

$$
\delta \mathcal{L}_{4 F}^{d=6}=-\frac{1}{M_{\Delta}^{2}} Y_{\Delta i j} Y_{\Delta \alpha \beta}^{\dagger}\left(\bar{L}_{\beta L} \gamma_{\mu} L_{L i}\right)\left(\bar{L}_{\alpha L} \gamma^{\mu} L_{j L}\right)
$$

Usando $\left(T^{i}\right)_{\alpha \beta}=-i \epsilon_{i \alpha \beta}, \delta \mathcal{L}_{\phi D}^{d=6}$ pode ser escrita na forma

$$
\delta \mathcal{L}_{\phi D}^{d=6}=4 \frac{\left|u_{\Delta}\right|^{2}}{M_{\Delta}^{4}}\left(\phi^{\dagger} \phi\right)\left[\left(D^{\mu} \phi\right)^{\dagger}\left(D_{\mu} \phi\right)\right]+4 \frac{\left|u_{\Delta}\right|^{2}}{M_{\Delta}^{4}}\left[\phi^{\dagger} D^{\mu} \phi\right]^{\dagger}\left[\phi^{\dagger} D_{\mu} \phi\right],
$$

onde a derivada covariante em (3.56) é expressada em termos das matrizes de Pauli e a hipercarga do Higgs $\left(Y_{H}=1\right)^{4}$,

$$
D_{\mu}=\partial_{\mu}-i g \frac{\tau_{a}}{2} W_{\mu}^{a}-i \frac{g^{\prime}}{2} B_{\mu}
$$

\footnotetext{
${ }^{4} \mathrm{~A}$ derivada covariante (3.57) é a mesma que aparece no potencial do Higgs, equação $(2.30)$.
} 


\subsubsection{Esquema de Renormalização}

Trabalhamos no Z-scheme [32] o qual usa como parâmetros de entrada os valores da constante de estrutura $\alpha$, a constante do Fermi $G_{F}$ e a massa do bóson $Z$ determinados experimentalmente. Produto das novas interações do modelo seesaw tipo II dois parâmetros são modificados: a massa do bóson Z e a constante de Fermi.

Os termos de massa para os bóson $Z$ e $W$ originam-se do termo cinético do campo de Higgs $\left(D^{\mu} \phi\right)^{\dagger}\left(D_{\mu} \phi\right)$ na lagrangiana do Higgs (2.19). Na equação (3.56), pode-se observar que este termo aparece quando o dubleto de Higgs adquire um VEV não nulo, $v / \sqrt{2}$, o termo $4 \frac{\left|u_{\Delta}\right|^{2}}{M_{\Delta}^{4}}\left(\phi^{\dagger} \phi\right)$ obtém o valor de $2 \frac{v^{2}\left|u_{\Delta}\right|^{2}}{M_{\Delta}^{4}}$ o qual indica que $M_{Z}^{2}$ é $M_{W}^{2}$ são corrigidos pelo fator $2 \frac{v^{2}\left|u_{\Delta}\right|^{2}}{M_{\Delta}^{4}} M_{Z, W}^{2}$, respectivamente. Além disso, o segundo termo em (3.56) apenas modifica o termo $M_{Z}^{2}$ da mesma forma. Assim, $M_{Z}^{2}$ e $M_{W}^{2}$ obtém uma correções $\delta M_{Z}^{2}$ e $\delta M_{W}^{2}$ dadas por:

$$
\begin{gathered}
\frac{\delta M_{Z}^{2}}{M_{Z}^{2}}=4 v^{2} \frac{\left|u_{\Delta}\right|^{2}}{M_{\Delta}^{4}}, \\
\frac{\delta M_{W}^{2}}{M_{W}^{2}}=2 v^{2} \frac{\left|u_{\Delta}\right|^{2}}{M_{\Delta}^{4}} .
\end{gathered}
$$

No MP a constante de Fermi é definida como o coeficiente em

$$
-\frac{4 G_{F}}{\sqrt{2}}\left(\bar{l}_{L e} \gamma_{u} \nu_{e}\right)\left(\bar{\nu} \gamma^{u} l_{L u}\right)
$$

usando $L_{\alpha L}=\left(\begin{array}{c}\nu_{\alpha L} \\ L_{\alpha L}\end{array}\right)$ na lagrangiana $\delta \mathcal{L}_{4 F}^{d=6}$ em (3.55), esta lagrangiana gera o o seguinte termo

$$
-\frac{4}{\sqrt{2}} \frac{1}{\sqrt{2} M_{\Delta}^{2}}\left|Y_{\Delta e \mu}\right|^{2}\left(\bar{l}_{L e} \gamma_{u} \nu_{e}\right)\left(\bar{\nu} \gamma^{u} l_{L u}\right),
$$

assim o coeficiente $\frac{4}{\sqrt{2}} \frac{1}{\sqrt{2} M_{\Delta}^{2}}\left|Y_{\Delta e \mu}\right|^{2}$ em (3.61) gera um deslocamento para a constante de Fermi com respeito à sua definição no $\mathrm{MP}, G_{F}^{M P}=1 /\left(\sqrt{2} v^{2}\right)$. Este deslocamento é dado por:

$$
\delta G_{F}=\frac{1}{\sqrt{2} M_{\Delta}^{2}}\left|Y_{\Delta e \mu}\right|^{2} .
$$




$$
G_{F}=G_{F}^{M P}+\delta G_{F} .
$$

O autoacoplamento quártico $\lambda$ na definição do potencial do Higgs, equação (2.20) é também modificado produto do operador de dimensão $d=4$, equação (3.47), gerando um deslocamento $\delta \lambda$ definido por

$$
\delta \lambda=-2 \frac{\left|u_{\Delta}\right|^{2}}{M_{\Delta}^{2}} .
$$

Como o operador $\delta \mathcal{L}_{4 \phi}^{d=6}$ em (3.53) é dado por $2\left(\lambda_{3}+\lambda_{5}\right) \frac{\left|u_{\Delta}\right|^{2}}{M_{\Delta}^{4}}|\phi|^{6}$ este modifica o potencial do Higgs definido em (2.20), junto como o deslocamento $\delta \lambda$ em (3.64) o potencial de higgs é dado por

$$
V=-\mu_{\phi}^{2}|\phi|^{2}+(\lambda+\delta \lambda)|\phi|^{4}+2\left(\lambda_{3}+\lambda_{5}\right) \frac{\left|u_{\Delta}\right|^{2}}{M_{\Delta}^{4}}|\phi|^{6} .
$$

Este novo potencial de Higgs gera um deslocamento no VEV do campo de Higgs dado por

$$
\delta v^{2}=-3 v^{4} \frac{2\left|u_{\Delta}\right|^{2}}{M_{\Delta}^{4}} \frac{\left(\lambda_{3}+\lambda_{5}\right)}{(\lambda+\delta \lambda)} .
$$

\subsection{Seesaw Tipo III}

Considere-se agora o MP estendido pela adição de $n$ tripletos de férmions de $S U(2)_{L}$ com $Y=0$, denotados por $\overrightarrow{\Sigma_{n}}=\left(\Sigma_{1}, \Sigma_{2}, \Sigma_{3}\right)_{n}$, onde $\Sigma_{1}, \Sigma_{2}$ e $\Sigma_{3}$ são suas três componentes $S U(2)_{L}$. Estando $\vec{\Sigma}$ na representação adjunta do grupo de gauge, seu termo de massa de Majorana é invariante de gauge e suas interações são descritas pela lagrangiana [35]

$\mathcal{L}_{\Sigma}=\overrightarrow{i \overrightarrow{\Sigma_{n R}}} D \overrightarrow{\Sigma_{n R}}-\left[\overrightarrow{L_{L}} \vec{\tau} \tilde{\phi} Y_{n \Sigma}^{\dagger} \overrightarrow{\Sigma_{n R}}+\overrightarrow{\Sigma_{n R}} Y_{n \Sigma} \widetilde{\phi}^{\dagger} L_{L}\right]-\frac{1}{2}\left[\overrightarrow{\Sigma_{n R}} M_{\Sigma} \overrightarrow{\Sigma_{m R}^{c}}+h . c\right]$.

Nesta equação, a derivada covariante é dada pelas equações (3.38) e (3.39) e as três componentes de $S U(2)_{L}$ têm a mesma massa de Majorana. Neste trabalho considera-se a base na qual $M_{\Sigma}$ é uma matriz diagonal no espaço de gerações. O acoplamento de Yukawa $Y_{\Sigma}$ em (3.67) é, em geral, uma matriz 
complexa no espaço de gerações. Definindo $\Sigma=\Sigma_{R}+\Sigma_{R}^{c}$, a lagrangiana em (3.67) pode ser escrita como:

$$
\begin{aligned}
\mathcal{L}_{\Sigma} & =\frac{1 \overrightarrow{\vec{\Sigma}}}{2}\left(i \not D-M_{\Sigma}\right) \vec{\Sigma} \\
& -\frac{1}{2} \bar{\Sigma}\left[Y_{\nu} \widetilde{\phi}^{\dagger} \vec{\tau} L_{L}+Y_{\Sigma}^{*} \phi^{\dagger} \vec{\tau} \widetilde{L}_{L}\right]-\frac{1}{2}\left[\overline{L_{L}} \vec{\tau} \widetilde{\phi} Y_{\Sigma}^{\dagger}+\overline{\widetilde{L}_{L}} \vec{\tau} \phi Y_{\Sigma}^{T}\right] \vec{\Sigma}
\end{aligned}
$$

onde $\widetilde{L} \equiv i \tau_{2} L^{c}$. Analogamente como acontece no modelo seesaw tipo I, é e se esperar a existência de operadores de dimensões $d=5$ e $d=6$, quando procedemos da mesma forma como no modelo seesaw tipo I. Resolvendo as equações de movimento

$$
\left.\frac{\delta S}{\delta \Sigma(x)}\right|_{\Sigma_{0}}=0,\left.\quad \frac{\delta S}{\delta \bar{\Sigma}(x)}\right|_{\Sigma_{0}}=0
$$

a solução clássica resulta

$$
\overrightarrow{\Sigma_{0}}=\frac{1}{i \not D-M_{\Sigma}}\left[Y_{\nu} \widetilde{\phi}^{\dagger} \vec{\tau} L_{L}+Y_{\Sigma}^{*} \phi^{\dagger} \vec{\tau} \widetilde{L}_{L}\right]
$$

como $\mathcal{L}_{\Sigma}$ tem termos até segunda ordem em $\Sigma$, em analogia com o modelo tipo I a ação efetiva é dada por

$$
S_{e f f}=S_{M P}+S_{N}\left[\Sigma_{0}\right]
$$

onde

$$
\begin{array}{r}
S_{N}\left[\Sigma_{0}\right]=\int d^{4} x \mathcal{L}_{\Sigma_{0}}=-\int d^{4} x \frac{1}{2}\left[\overline{L_{L}} \vec{\tau} \widetilde{\phi} Y_{\Sigma}^{\dagger}+\overline{\widetilde{L}_{L}} \vec{\tau} \phi Y_{\Sigma}^{T}\right] \\
\left(\frac{1}{i \not D-M_{\Sigma}}\right)\left[Y_{\nu} \widetilde{\phi}^{\dagger} \vec{\tau} L_{L}+Y_{\Sigma}^{*} \phi^{\dagger} \vec{\tau} \widetilde{L}_{L}\right]
\end{array}
$$

\subsubsection{Operador de dimensão $d=5$}

Considerando $D_{\mu}$ no lugar de $\partial_{\mu}$ na equação (3.15), e a equação (3.72) o operador de dimensão $d=5$ da lagrangiana efetiva para o modelo seesaw tipo III é dado por

$$
\delta \mathcal{L}^{d=5}=\frac{1}{2}\left(Y_{\Sigma}^{T} \frac{1}{M_{\Sigma}} Y_{\Sigma}\right)_{\alpha \beta}\left(\widetilde{\widetilde{L}_{\alpha L}} \vec{\tau} \phi\right)\left(\widetilde{\phi}^{\dagger} \vec{\tau} L_{\beta L}\right)+h . c
$$


Usando a identidade $\left(\tau_{i}\right)_{a b}\left(\tau_{i}\right)_{c d}=2\left(\delta_{b c} \delta_{a d}-\frac{1}{2} \delta_{a b} \delta_{c d}\right)$, a equação (3.73) pode ser escrita como

$$
\delta \mathcal{L}^{d=5}=\frac{1}{2}\left(Y_{\Sigma}^{T} \frac{1}{M_{\Sigma}} Y_{\Sigma}\right)_{\alpha \beta}\left(\overline{L_{\alpha L}^{c}} \widetilde{\phi}^{*}\right)\left(\widetilde{\phi}^{\dagger} L_{\beta L}\right)+\text { h.c. }
$$

Comparando esta última expressão com a equação (3.16), o coeficiente do operador $d=5$ é dado pela matriz

$$
c_{\alpha \beta}^{d=5}=\left(Y_{\Sigma}^{T} \frac{1}{M_{\Sigma}} Y_{\Sigma}\right)_{\alpha \beta}
$$

e analogamente, como o modelo seesaw tipo I, o operador de dimensão $d=5$ gera massa de Majorana para os neutrinos, com a matriz de massa da forma

$$
m_{\alpha \beta}=-\frac{v^{2}}{2} c_{\alpha \beta}^{d=5}=-\frac{v^{2}}{2}\left(Y_{\Sigma}^{T} \frac{1}{M_{\Sigma}} Y_{\Sigma}\right)_{\alpha \beta}
$$

\subsubsection{Operador de dimensão $d=6$}

Considerando $D_{\mu}$ no lugar de $\partial_{\mu}$ na equação (3.15) e a equação (3.72), o operador de dimensão $d=6$ da lagrangiana efetiva para o modelo seesaw tipo III é dado por

$$
\delta \mathcal{L}^{d=6}=\frac{1}{2}\left[\overline{L_{L}} \vec{\tau} \widetilde{\phi} Y_{\Sigma}^{\dagger}+\widetilde{L}_{L} \vec{\tau} \phi Y_{\Sigma}^{T}\right]\left(\frac{i \not D}{M_{\Sigma}^{\dagger} M_{\Sigma}}\right)\left[Y_{\nu} \widetilde{\phi}^{\dagger} \vec{\tau} L_{L}+Y_{\Sigma}^{*} \phi^{\dagger} \vec{\tau} \widetilde{L}_{L}\right]
$$

Usando (3.21) a equação (3.77) pode ser escrita como

$$
\delta \mathcal{L}^{d=6}=c_{\alpha \beta}^{d=6}\left(\overline{L_{\alpha L}} \vec{\tau} \widetilde{\phi}\right) i \not D\left(\widetilde{\phi}^{\dagger} \vec{\tau} L_{\beta L}\right),
$$

onde o coeficiente do operador de dimensão $d=6$ e dado por

$$
c_{\alpha \beta}^{d=6}=\left(Y_{\Sigma}^{\dagger} \frac{1}{M_{\Sigma}^{\dagger} M_{\Sigma}} Y_{\Sigma}\right)_{\alpha \beta} .
$$

Pode-se observar o paralelismo entre o resultado do contexto seesaw tipo III e o modelo seesaw tipo I, equações (3.17) e (3.22-3.23).

Quando o dubleto de Higgs adquire um VEV não nulo, o operador de dimensão $d=6$ corrige o termo de energia cinética para os neutrinos e os 
léptons, assim como seus acoplamentos com o bóson $W$. Depois da quebra espontânea da simetria, o operador de dimensão $d=6$ escreve-se como

$$
\begin{aligned}
\delta \mathcal{L}^{d=6} & =2 \epsilon_{\alpha \beta}^{\Sigma} \overline{l_{\alpha L}} i \not \partial l_{\beta L}+\epsilon_{\alpha \beta}^{\Sigma} \overline{\nu_{\alpha L}} i \not \partial \nu_{\beta L}-2 e \epsilon_{\alpha \beta}^{\Sigma} \overline{l_{\alpha L}} \gamma^{\mu} l_{\beta L} A_{\mu} \\
& -\frac{g}{2 \cos \theta}\left[4 e \epsilon_{\alpha \beta}^{\Sigma} \overline{l_{\alpha L}} \gamma^{\mu} l_{\beta L}-4 e \sin ^{2} \theta_{W} \epsilon_{\alpha \beta}^{\Sigma} \overline{l_{\alpha L}} \gamma^{\mu} l_{\beta L}\right] Z_{\mu}
\end{aligned}
$$

onde

$$
\epsilon_{\alpha \beta}^{\Sigma}=\frac{v^{2}}{2} c_{\alpha \beta}^{d=6}
$$

Com (3.80) a parte da lagrangiana efetiva referente aos léptons é, na base de sabor

$$
\begin{aligned}
\mathcal{L}_{\text {léptons }}^{d \leq 6} & =i \overline{\nu_{\alpha L}} \not \partial\left(\delta_{\alpha \beta}+\epsilon_{\alpha \beta}^{\Sigma}\right) \nu_{\beta L}-i \overline{\bar{l}_{\alpha L}}\left(\delta_{\alpha \beta}+2 \epsilon_{\alpha \beta}^{\Sigma}\right) \not l_{\beta L} \\
& -\frac{1}{2}\left(\overline{\nu_{\alpha L}^{c}} m_{\nu \alpha \beta} \nu_{\alpha L}+h . c\right)-\overline{l_{\alpha L}} m_{l \alpha \beta} \nu_{\beta L}+\mathcal{L}^{(C C)}+\mathcal{L}^{(N C)}
\end{aligned}
$$

com

$$
\begin{aligned}
\mathcal{L}_{I, L}^{(C C)} & =\frac{g}{\sqrt{2}} \bar{l}_{\alpha L} \gamma^{\mu}\left(1+2 \epsilon^{\Sigma}\right)_{\alpha \beta} \nu_{\beta L} W_{\mu}^{-}+h . c, \\
\mathcal{L}_{I, L}^{(N C)} & =\frac{g}{2 \cos \theta_{W}}\left[\bar{\nu}_{L} \gamma^{\mu} \nu_{L}-\bar{l}_{\alpha L} \gamma^{\mu}\left(1+4 \epsilon^{\Sigma}\right)_{\alpha \beta} l_{\beta L}+2 \sin ^{2} \theta_{W} \bar{l}_{\alpha L} \gamma^{\mu}\left(1+2 \epsilon^{\Sigma}\right)_{\alpha \beta} l_{\beta L}\right. \\
& \left.+2 \sin ^{2} \theta_{W} \bar{l}_{\alpha R} \gamma^{\mu} l_{\alpha R}\right] Z_{\mu}-e \bar{l}_{\alpha L}\left(1+2 \epsilon^{\Sigma}\right)_{\alpha \beta} \gamma^{\mu} l_{\beta L} A_{\mu}-e \bar{l}_{\alpha R} \gamma^{u} l_{\alpha R} A_{u},
\end{aligned}
$$

Os campos para os léptons carregados e os neutrinos necessitam ser normalizados para ter um termo cinético canonicamente normalizado. Até $\mathcal{O}\left(1 / M_{\Sigma}^{2}\right)$, isto é, linear nos parâmetros de $\epsilon^{\Sigma}$, as redefinições

$$
\begin{aligned}
\nu_{\alpha L} & =\left(1-\frac{\epsilon^{\Sigma}}{2}\right)_{\alpha \beta} \nu_{\beta L}^{\prime}, \\
l_{\alpha L} & =\left(1-\epsilon^{\Sigma}\right)_{\alpha \beta} l_{\beta L}^{\prime},
\end{aligned}
$$

resultam numa lagrangiana na base de sabor que, até $\mathcal{O}\left(1 / M_{\Sigma}^{2}\right)$, toma a forma 5

$$
\begin{aligned}
\mathcal{L}_{\text {léptons }}^{d \leq 6} & =i \overline{\nu_{\alpha L}} \not \partial \nu_{\alpha L}-i \overline{l_{\alpha L}} \not l_{\alpha L}-\frac{1}{2}\left(\overline{\nu_{\alpha L}^{c}} m^{\prime}{ }_{\nu \alpha \beta} \nu_{\alpha L}+h . c\right)-\overline{l_{\alpha L}} m_{l \alpha \beta}^{\prime} \nu_{\beta L} \\
& +\mathcal{L}^{(C C)}+\mathcal{L}^{(N C)}
\end{aligned}
$$

\footnotetext{
${ }^{5}$ Onde foram retiradas as linhas sobre os campos.
} 
onde $m^{\prime}{ }_{\nu}=\left(1-\left(\epsilon^{\Sigma}\right)^{T} / 2\right) m_{\nu}\left(1-\epsilon^{\Sigma} / 2\right), m_{l}^{\prime}=\left(1-\epsilon^{\Sigma}\right) m_{l} \mathrm{e}$

$$
\begin{aligned}
\mathcal{L}_{I, L}^{(C C)}= & \frac{g}{\sqrt{2}} \bar{l}_{\alpha L} \gamma^{\mu}\left(1+\frac{\epsilon^{\Sigma}}{2}\right)_{\alpha \beta} \nu_{\beta L} W_{\mu}^{-}+h . c \\
\mathcal{L}_{I, L}^{(N C)}= & \frac{g}{2 \cos \theta_{W}}\left[\bar{\nu}_{\alpha L} \gamma^{\mu}\left(1-\epsilon^{\Sigma}\right)_{\alpha \beta} \nu_{\beta L}-\bar{l}_{\alpha L} \gamma^{\mu}\left(1+2 \epsilon^{\Sigma}\right)_{\alpha \beta} l_{\beta L}\right. \\
& \left.+2 \sin ^{2} \theta_{W} \bar{l}_{\alpha} \gamma^{\mu} l_{\beta}\right] Z_{\mu}-e \bar{l}_{\alpha} \gamma^{\mu} l_{\alpha} A_{\mu} .
\end{aligned}
$$

Analogamente ao modelo seesaw tipo I, pode-se rodar a base para a qual as matrizes de massa são diagonais e o termo cinético é canonicamente normalizado. Tal base é definida como

$$
\begin{aligned}
\nu_{i} & =V_{i \alpha}^{e f f} \nu_{\alpha L}+V_{i \alpha}^{e f f *} \nu_{\alpha L}^{c}, \text { com } \quad V^{e f f}=U^{\nu+}\left(1+\frac{\epsilon^{\Sigma}}{2}\right), \\
l_{\alpha L} & =k_{\alpha \beta}^{e f f} l_{\beta L}, \text { com } \quad k^{e f f}=U_{L}^{l+}\left(1+\epsilon^{\Sigma}\right), \\
l_{\alpha R} & =\left(U_{R}^{l+}\right)_{\alpha \beta} l_{\beta R},
\end{aligned}
$$

onde $U$ e $U^{l}$ são as matrizes que diagonalizam os termos de massa dos neutrinos e dos léptons carregados, respectivamente, $m_{\nu}^{\text {diag }} \equiv U^{\nu T} m_{\nu} U^{\nu}$ e $m_{l}^{\text {diag }} \equiv$ $U_{R}^{l+} m_{l}\left(1-\epsilon^{\Sigma}\right) U_{L}^{l}$. Em termos dos autoestados de massa, a lagrangiana para os léptons é dada por

$$
\mathcal{L}_{\text {léptons }}^{d \leq 6}=\frac{1}{2} i \overline{\nu_{i}}\left(\not \partial-m_{\nu i}^{\text {diag }}\right) \nu_{i}-\overline{l_{\alpha L}}\left(i \not \partial-m_{l \alpha}^{\text {diag }}\right) l_{\alpha L}+\mathcal{L}^{(C C)}+\mathcal{L}^{(N C)}
$$

onde, nesta base de massa, as lagrangianas $\mathcal{L}^{(C C)}$ e $\mathcal{L}^{(N C)}$, escrevem-se como

$$
\begin{aligned}
\mathcal{L}_{I, L}^{(C C)} & =\frac{g}{\sqrt{2}} \bar{l}_{\alpha L} \gamma^{u} N_{\alpha i} \nu_{i L} W_{u}^{-}+h . c \\
\mathcal{L}_{I, L}^{(N C)} & =\frac{g}{2 \cos \theta_{W}}\left[\bar{\nu}_{i L} \not Z\left(N^{\dagger} N\right)_{i j}^{-1} \nu_{j_{L}}-\bar{l}_{\alpha L} \not Z\left(N N^{\dagger}\right)_{\alpha \beta}^{2} l_{\beta L}+2 \sin ^{2} \theta_{W} \bar{l}_{\alpha} \not Z l_{\alpha}\right] \\
& -e \bar{l}_{\alpha} A l_{\alpha},
\end{aligned}
$$

com

$$
N \equiv \Omega U_{L}^{l+}\left(1+\frac{\epsilon^{\Sigma}}{2}\right) U^{\nu}
$$

onde, de novo $\Omega \equiv \operatorname{diag}\left(e^{i w_{1}}, e^{i w_{2}}, e^{i w_{3}}\right)$ reabsorve as três fases não físicas na definição de léptons carregados, como é usual. Em termos da matriz $N$, as correntes neutras e carregadas definidas em (3.28) são

$$
j_{W, L}^{\mu}=2 \bar{l}_{\alpha L} \gamma^{\mu} N_{\alpha i} \nu_{i L}
$$



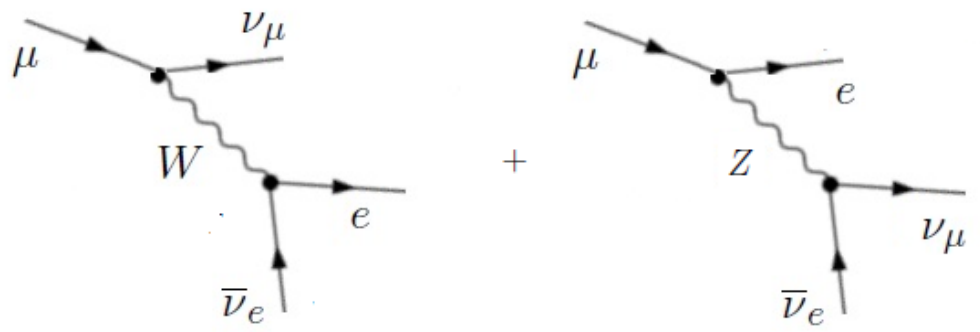

Figura 3.1: Diagramas que contribuem ao processo $\mu \rightarrow \nu_{\mu} e \bar{\nu}_{e}$. O primeiro diagrama é produto da interação carregada (3.91) e o segundo termo produto da interação de léptons carregados com o bóson $Z$, equação (3.93).

$$
\begin{aligned}
& J_{Z, L}^{\mu}(\text { neutrinos })=\bar{\nu}_{i} \gamma^{\mu}\left(N^{\dagger} N\right)_{i j}^{-1} \nu_{j L}, \\
& J_{Z, L}^{\mu}(\text { leptons })=\bar{l}_{\alpha L} \gamma^{\mu}\left(N N^{\dagger}\right)_{\alpha \beta}^{2} l_{\beta L}
\end{aligned}
$$

$\mathrm{Na}$ base de sabor, $U_{L}^{l+}$ e $\Omega$ são iguais à matriz identidade e a matriz $U^{\nu}=U_{P M N S}$, assim a matriz não unitária $N$ é dada por

$$
N=\left(1+\frac{\epsilon^{\Sigma}}{2}\right) U_{P M N S}
$$

enquanto que os acoplamentos com o bóson $Z$ adquirem também uma matriz de mistura não unitária $N N^{\dagger}=\left(1+\epsilon^{\Sigma}\right)$ e $N^{\dagger} N=U_{P M N S}^{\dagger}\left(1+\epsilon^{\Sigma}\right) U_{P M N S}$. Estas expressões podem ser comparadas com suas equivalentes para o modelo seesaw tipo I, equação (3.32). Qualquer que seja a base, as correntes nas equações (3.91)-(3.93) podem também ser comparadas com suas correspondentes para o modelo seesaw tipo I, equações (3.30) e (3.31).

Um padrão não-unitário de mistura apareceu em ambos modelos. Embora a modificação dos acoplamentos $Z$-neutrino sejam diferentes, uma matriz de mistura não unitária de sabor está também presente nas interações dos léptons carregados com o bóson $Z$, o qual dá origem a violação de sabor no setor de léptons carregados, e permite processos como $\mu \rightarrow 3 e$ e $\tau \rightarrow 3 l$. 
Uma importante consequência da mudança de sabor nas interações dos léptons com os bósons $Z$ e $W$ é sua contribuição para o decaimento $\mu^{-} \rightarrow$ $\nu_{\mu} e^{-} \bar{\nu}_{e}$ (figura (3.1)), que modifica a definição de $G_{F}$, como segue

$$
G_{F}=G_{F}^{M P} \sqrt{\left(N N^{\dagger}\right)_{e e}\left(N N^{\dagger}\right)_{\mu \mu}+\frac{3}{4}\left[\left(N N^{\dagger}\right)_{e \mu}^{2}\right]^{2}},
$$

onde o primeiro termo em (3.95) é a contribuição produto da interação carregada (3.91) tal como acontece no modelo seesaw tipo I. O segundo termo em (3.95) é produto da mistura de léptons carregados que acontece na interação com o bóson $Z$ da equação (3.93). Como ainda não se tem observado processos que violem sabor leptônico, é de esperar-se que a contribuição deste último termo, $\left(\frac{3}{4}\left[\left(N N^{\dagger}\right)_{e \mu}^{2}\right]^{2}\right)$, seja pequena comparada com a contribuição produto da interação carregada $\left(\left(N N^{\dagger}\right)_{e e}\left(N N^{\dagger}\right)_{\mu \mu}\right)$. Assim,

$$
G_{F} \approx G_{F}^{M P} \sqrt{\left(N N^{\dagger}\right)_{e e}\left(N N^{\dagger}\right)_{\mu \mu}} .
$$

Por último, em analogia com o caso de seesaw tipo I o resto dos parâmetros da lagrangiana coincidem com o tratamento padrão. É importante destacar que os supostos desvios da unitariedade da matriz de mistura leptônica podem ser relacionados com o coeficiente de operador $d=6$

$$
\left|\left(N N^{\dagger}\right)_{\alpha \beta}-1\right|=\frac{v^{2}}{2}\left|c^{d=6}\right|_{\alpha \beta}=\frac{v^{2}}{2}\left(Y_{\Sigma}^{\dagger} \frac{1}{M_{\Sigma}^{\dagger} M_{\Sigma}} Y_{\Sigma}\right)_{\alpha \beta} .
$$




\section{Capítulo 4}

\section{Fenomenologia}

Neste capítulo, vamos impor restrições sobre a matriz $Y_{\Delta}$, no caso do modelo seesaw tipo II e sobre a matriz $N N^{\dagger}$, no caso do modelo seesaw tipo III e seesaw tipo I. Para tanto usaremos os limites experimentais para as razões de decaimento de processos que violam número leptônico como $\operatorname{Br}\left(\mu^{-} \rightarrow\right.$ $\left.e^{+} e^{-} e^{-}\right)<10^{-12}, \operatorname{Br}\left(\tau^{-} \rightarrow e^{+} e^{-} e^{-}\right)<2,7 \times 10^{-8}$ e $\operatorname{Br}\left(\tau^{-} \rightarrow \mu^{+} \mu^{-} \mu^{-}\right)<$ $2,1 \times 10^{-8}$ [22]. Além disso, levaremos em conta processos até um loop, no qual se tem que usar limites experimentais como $\operatorname{Br}\left(\tau^{-} \rightarrow e^{-} \nu_{\tau} \bar{\nu}_{e}\right)=17,83 \%$, $\operatorname{Br}\left(\mu^{-} \rightarrow e \gamma\right)<2,4 \times 10^{-12}, \operatorname{Br}\left(\tau^{-} \rightarrow \mu \gamma\right)<3,3 \times 10^{-8}$ e $\operatorname{Br}\left(\tau^{-} \rightarrow e \gamma\right)<$ $4,4 \times 10^{-8}[22]$.

\subsection{Seesaw Tipo II}

Usando os valores experimentais para os parâmetros $\alpha, G_{F}$ e $M_{Z}$ definidos no $Z$-scheme no capítulo 3 , obteremos os desvios dos parâmetros $\rho$ e $M_{W}$ com respeito ao MP.

\subsection{1 $M_{W}$ e o parâmetro $\rho$}

Primeiramente consideremos apenas a modificação do parâmetro $\rho$ obtida de transições hadrônicas, ou seja, sem considerar a modificação da constante de Fermi em (3.62). Assim o parâmetro $\rho$ definido em (2.50) tem um deslocamento produto de $\delta M_{Z}^{2}$ e $\delta M_{W}^{2}$ dadas pelas equações (3.58) e (3.59), 
respetivamente. Assim

$$
\rho=\frac{M_{W}^{2}}{\cos ^{2} \theta_{W} M_{Z}^{2}}=1 \rightarrow \rho=\frac{M_{W}^{2}}{\cos ^{2} \theta_{W} M_{Z}^{2}} \frac{\left(1+2 v^{2} \frac{\left|u_{\Delta}\right|^{2}}{M_{\Delta}^{4}}\right)}{\left(1+4 v^{2} \frac{\left|u_{\Delta}\right|^{2}}{M_{\Delta}^{4}}\right)} \approx\left(1-2 v^{2} \frac{\left|u_{\Delta}\right|^{2}}{M_{\Delta}^{4}}\right),
$$

o qual gera o deslocamento ${ }^{1}$

$$
\delta \rho_{\text {had }}=-\frac{\sqrt{2}\left|\mu_{\Delta}\right|^{2}}{G_{F} M_{\Delta}^{4}} .
$$

Levando em conta o valor médio experimental do parâmetro $\rho[22]$ ( $\rho=$ $1.0002 \pm 0.0004)$, considerando apenas o domínio de contribuições hadrônicas, que não depende do acoplamento de Yukawa equação 4.2, obtém-se o limite

$$
\frac{\mu_{\Delta}}{M_{\Delta}^{2}}<[0.04 \pm 0.08](\mathrm{TeV})^{-1}
$$

Consideremos a massa do neutrino na equação (3.50) de ordem de $1 \mathrm{eV}$ e o acoplamento de Yukawa $Y_{\Delta} \sim \mathcal{O}(1)$, isto implica $\frac{\mu_{\Delta}}{M_{\Delta}^{2}} \sim 10^{-11}(\mathrm{TeV})^{-1}$ muito abaixo do limite em (4.3), enquanto o menor valor de $Y_{\Delta}$ que satisfaz (4.3) com a massa do neutrino da ordem de $1 \mathrm{eV}$ é $Y_{\Delta} \sim 10^{-7}$.

Agora consideremos $M_{W}$ definido no contexto do MP no $Z$-scheme [22]

$$
\left(M_{W}^{M P}\right)^{2}=\frac{M_{Z}^{2}}{2}\left[1+\sqrt{1-\frac{4 \pi \alpha}{\sqrt{2} G_{F} M_{Z}^{2}(1-\Delta r)}}\right],
$$

onde $\Delta r$ indica correções radiativas até um loop no MP. Como a lagrangiana $\delta \mathcal{L}_{4 F}^{d=6}$ modifica o valor de $G_{F}$ equação (3.62), considerando apenas a variação de $G_{F}$ na equação (4.4), obtemos um deslocamento na massa do bóson $W$,

$$
\delta M_{W}^{2}=\frac{M_{W}^{2}\left[M_{W}^{2}-M_{Z}^{2}\right]}{2 M_{W}^{2}-M_{Z}^{2}} \frac{\delta G_{F}}{G_{F}}=\frac{M_{W}^{2}\left[M_{W}^{2}-M_{Z}^{2}\right]\left|Y_{\Delta e \mu}\right|^{2}}{\left[2 M_{W}^{2}-M_{Z}^{2}\right] \sqrt{2} G_{F} M_{\Delta}^{2}} .
$$

O valor experimental muito bem conhecido da massa do $W$ permite obter o limite para o valor de $\left|Y_{\Delta e \mu}\right|$. Da diferença experimental e a predição no contexto do MP no $Z$-scheme $\left[M_{W}^{M P}=(80.4887 \pm 0.0515) \mathrm{GeV}\right]$

$$
\left|Y_{\Delta e \mu}\right|^{4} \leq[0.00023 \pm 0.00109]\left(M_{\Delta} / \mathrm{TeV}\right)^{4},
$$

que gera

$$
M_{\Delta} \geq 9 \mathrm{TeV}, \quad \text { para } Y_{\Delta} \sim \mathcal{O}(1)
$$

\footnotetext{
${ }^{1}$ Como não se considera o deslocamento $\delta G_{F}$ ainda se tem $2 v^{2}=\frac{G_{F}}{\sqrt{2}}$.
} 


\subsubsection{Decaimento de $\mu \rightarrow 3 e$ e $\tau \rightarrow 3 l$}

$\delta \mathcal{L}_{4 \bar{F}}^{d=6}$ na equação (3.55) induz acoplamento entre quatro férmions que pode levar a processos de violação de sabor. Pode-se observar que este operador não depende de $\mu_{\Delta}$. Como a constante de Fermi é modificada, isto altera o razão entre as larguras de decaimentos para léptons. As restrições pelos limites experimentais para tais processos são estudados nas referências [38][40].

Considerando os decaimentos $\mu \rightarrow 3 e$ e $\tau \rightarrow 3 l$, podemos obter limites sobre os coeficientes $c_{\alpha \beta \gamma \delta}^{4 F} \equiv \frac{1}{M_{\Delta}^{2}} Y_{\Delta \alpha \beta} Y_{\Delta \gamma \delta}^{\dagger}$ do operador $\delta \mathcal{L}_{4 F}^{d=6}$ na equação (3.55). Assim, para o caso do decaimento do $\mu \rightarrow 3 e$ obtém-se

$$
\operatorname{Br}\left(\mu^{-} \rightarrow e^{+} e^{-} e^{-}\right)=\frac{\Gamma\left(\mu^{-} \rightarrow e^{\dagger} e^{-} e^{-}\right)}{\Gamma\left(\mu^{-} \rightarrow e^{-} \nu_{\mu} \bar{\nu}_{e}\right)}=\frac{1}{G_{F}^{2}}\left[\frac{Y_{\Delta e \mu} Y_{\Delta e e}^{\dagger}}{M_{\Delta}^{4}}\right] .
$$

Analogamente para o caso do decaimento $\tau$ em três léptons carregados, podese mostrar que

$$
\begin{aligned}
\operatorname{Br}\left(\tau^{-} \rightarrow l_{i}^{+} l_{j}^{-} l_{j}^{-}\right) & =\frac{\Gamma\left(\tau^{-} \rightarrow l_{i}^{+} l_{j}^{-} l_{j}^{-}\right)}{\Gamma\left(\tau^{-} \rightarrow \nu_{\tau} l_{i} \bar{\nu}_{i}\right)} \operatorname{Br}\left(\tau^{-} \rightarrow \nu_{\tau} l_{i} \bar{\nu}_{i}\right) \\
& =\frac{1}{16 G_{F}^{2}}\left[\frac{Y_{\Delta \tau i} Y_{\Delta j j}^{\dagger}}{M_{\Delta}^{4}}\right] \operatorname{Br}\left(\tau^{-} \rightarrow \nu_{\tau} l_{i} \bar{\nu}_{i}\right)
\end{aligned}
$$

para algum $i, j$, enquanto

$$
\begin{aligned}
\operatorname{Br}\left(\tau^{-} \rightarrow l_{i}^{+} l_{j}^{-} l_{k}^{-}\right) & =\frac{\Gamma\left(\tau^{-} \rightarrow l_{i}^{\dagger} l_{j}^{-} l_{k}^{-}\right)}{\Gamma\left(\tau^{-} \rightarrow \nu_{\tau} l_{i} \bar{\nu}_{i}\right)} \operatorname{Br}\left(\tau^{-} \rightarrow \nu_{\tau} l_{i} \bar{\nu}_{i}\right) \\
& =\frac{1}{8 G_{F}^{2}}\left[\frac{Y_{\Delta \tau i} Y_{\Delta j k}^{\dagger}}{M_{\Delta}^{4}}\right] \operatorname{Br}\left(\tau^{-} \rightarrow \nu_{\tau} l_{i} \bar{\nu}_{i}\right)
\end{aligned}
$$

para algum $i, j, k$ com $j \neq k$. Usando os limites experimentais para a razões de decaimento fornecidos em [22], os valores de $\operatorname{Br}\left(\tau^{-} \rightarrow \nu_{\tau} e^{-} \bar{\nu}_{e}\right)=17,83 \%$, $\operatorname{Br}\left(\tau^{-} \rightarrow \nu_{\tau} \mu^{-} \bar{\nu}_{\mu}\right)=17,41 \%$ e $G_{F}=(1,16637 \pm 0,00001) \times 10^{-5}(\mathrm{GeV})^{-2}$, os limites para os correspondentes acoplamentos de Yukawa são fornecidos na tabela (4.1).

A não observação de transições que violem sabor leptônico, em particular para $\mu \rightarrow 3 e$, gera o menor limite para a escala do seesaw tipo II,

$$
\begin{aligned}
& M_{\Delta} \geq 294 \mathrm{TeV}, \quad \text { para } Y_{\Delta} \sim \mathcal{O}(1), \\
& M_{\Delta} \geq 3 \mathrm{TeV}, \quad \text { para } Y_{\Delta} \sim \mathcal{O}\left(10^{-2}\right) .
\end{aligned}
$$




\begin{tabular}{|c|c|c|}
\hline Restrições sobre & Processo & limite $\left[\times\left(\frac{M_{\Delta}}{1 \mathrm{TeV}}\right)^{2}\right]$ \\
\hline$\left|Y_{\Delta \mu e}\right|\left|Y_{\Delta e e}\right|$ & $\mu^{-} \rightarrow e^{+} e^{-} e^{-}$ & $<1,2 \times 10^{-5}$ \\
\hline$\left|Y_{\Delta \tau e}\right|\left|Y_{\Delta e e}\right|$ & $\tau^{-} \rightarrow e^{+} e^{-} e^{-}$ & $<1,8 \times 10^{-2}$ \\
\hline$\left|Y_{\Delta \tau \mu \mu}\right|\left|Y_{\Delta \mu \mu}\right|$ & $\tau^{-} \rightarrow \mu^{+} \mu^{-} \mu^{-}$ & $<1,6 \times 10^{-2}$ \\
\hline$\left|Y_{\Delta \tau \mu}\right|\left|Y_{\Delta \mu e}\right|$ & $\tau^{-} \rightarrow \mu^{+} \mu^{-} e^{-}$ & $<1,3 \times 10^{-2}$ \\
\hline$\left|Y_{\Delta \tau e}\right|\left|Y_{\Delta \mu \mu}\right|$ & $\tau^{-} \rightarrow e^{+} \mu^{-} \mu^{-}$ & $<1,4 \times 10^{-2}$ \\
\hline$\left|Y_{\Delta \tau e}\right|\left|Y_{\Delta \mu e}\right|$ & $\tau^{-} \rightarrow e^{+} e^{-} \mu^{-}$ & $<1,0 \times 10^{-2}$ \\
\hline$\left|Y_{\Delta \tau \mu}\right|\left|Y_{\Delta e e}\right|$ & $\tau^{-} \rightarrow \mu^{+} e^{-} e^{-}$ & $<1,4 \times 10^{-2}$ \\
\hline$\left|\sum_{l} Y_{\Delta l \mu}^{\dagger} Y_{\Delta e l}\right|$ & $\mu^{-} \rightarrow e \gamma$ & $<4,7 \times 10^{-3}$ \\
\hline$\left|\sum_{l} Y_{\Delta l \tau}^{\dagger} Y_{\Delta e l}\right|$ & $\tau^{-} \rightarrow \mu \gamma$ & $<1,05$ \\
\hline$\left|\sum_{l} Y_{\Delta l \tau}^{\dagger} Y_{\Delta \mu l}\right|$ & $\tau^{-} \rightarrow e \gamma$ & $<8,4 \times 10^{-1}$ \\
\hline
\end{tabular}

Tabela 4.1: Restrições sobre $Y_{\Delta \alpha \beta}$ oriundas do decaimento de léptons carregados e de processos até um loop $l_{1} \rightarrow l_{2} \gamma$.

Enquanto, para processos como $\tau \rightarrow 3 e$, o limite para a escala seesaw tipo II, é

$$
M_{\Delta} \geq 8 \mathrm{TeV}, \quad \text { para } \quad Y_{\Delta} \sim \mathcal{O}(1) .
$$

\subsection{3 $\quad l_{1} \rightarrow l_{2} \gamma$}

Para avaliar os limites impostos pelo estudo de decaimentos radiativos $l_{1} \rightarrow$ $l_{2} \gamma$, eles não podem ser obtidos dos operadores de dimensão $d=6$, pelo 

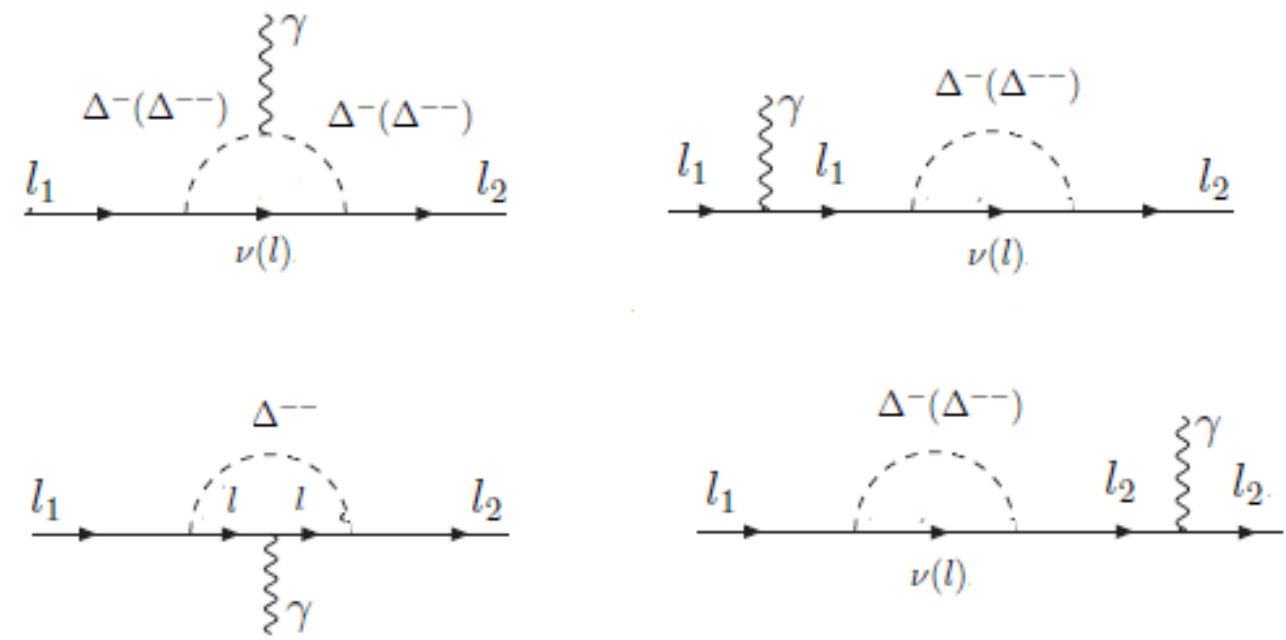

Figura 4.1: Diagramas que contribuem aos processos $l_{1} \rightarrow l_{2} \gamma$ com os tripletos carregados $\Delta^{-}$e $\Delta^{--}$como estados intermediários [36]

motivo de serem processos até um loop. Para tanto trabalharemos com a a lagrangiana da teoria completa em função das componentes carregadas

$$
\Delta^{++} \equiv \frac{\left(\Delta_{1}-i \Delta_{2}\right)}{\sqrt{2}}, \Delta^{+} \equiv \Delta_{3}, \quad \Delta^{0} \equiv \frac{\left(\Delta_{1}+i \Delta_{2}\right)}{\sqrt{2}}
$$

sendo que $\mathcal{L}_{\Delta}$ se escreve como

$$
\begin{aligned}
\mathcal{L}_{\Delta} & =D_{\mu} \Delta^{0 *} D^{\mu} \Delta^{0}+D_{\mu} \Delta^{+} D^{\mu} \Delta^{-}+D_{\mu} \Delta^{++} D^{\mu} \Delta^{--} \\
& +\left[\left(\overline{\nu_{L}^{c}} Y_{\Delta}^{\dagger} l_{L}\right) \Delta^{-}+\left(\overline{l_{L}^{c}} Y_{\Delta}^{\dagger} \nu_{L}\right) \Delta^{-}+\sqrt{2}\left(\overline{l_{L}^{c}} Y_{\Delta}^{\dagger} l_{L}\right) \Delta^{--}-\sqrt{2}\left(\overline{\nu_{L}^{c}} Y_{\Delta}^{\dagger} \nu_{L}\right) \Delta^{0}+h . c\right] \\
& +\left[u_{\Delta}\left(2 \phi^{0} \phi^{+} \Delta^{-}+\sqrt{2} \phi^{0} \phi^{0} \Delta^{0 *}-\sqrt{2} \phi^{+} \phi^{0} \Delta^{--}\right)+h . c\right] \\
& -M_{\Delta}^{2}\left(\Delta^{0 *} \Delta^{0}+\Delta^{-} \Delta^{+}+\Delta^{--} \Delta^{++}\right)-\frac{\lambda_{2}}{2}\left(\Delta^{0 *} \Delta^{0}+\Delta^{-} \Delta^{+}+\Delta^{--} \Delta^{++}\right)^{2} \\
& -\lambda_{3}\left(\phi^{-} \phi^{+}+\phi^{0 *} \phi^{0}\right)\left(\Delta^{0 *} \Delta^{0}+\Delta^{-} \Delta^{+}+\Delta^{--} \Delta^{++}\right)-\lambda_{4}\left[\frac{1}{2}\left(\Delta^{0 *} \Delta^{0}\right)^{2}+\frac{1}{2}\left(\Delta^{++} \Delta^{--}\right)^{2}\right. \\
& \left.+\Delta^{++} \Delta^{--}\left(\Delta^{+} \Delta^{-}-\Delta^{0 *} \Delta^{0}\right)+\Delta^{+} \Delta^{-} \Delta^{0 *} \Delta^{0}-\left(\Delta^{++} \Delta^{-} \Delta^{-} \Delta^{0}+h . c\right)\right] \\
& -\lambda_{5}\left[\left(\Delta^{++} \Delta^{--}-\Delta^{0 *} \Delta^{0}\right)\left(\phi^{+} \phi^{-}-\phi^{0 *} \phi^{0}\right)-\sqrt{2} \phi^{-} \phi^{0}\left(\Delta^{++} \Delta^{-}-\Delta^{0 *} \Delta^{+}\right)+h . c\right],
\end{aligned}
$$


onde $\Delta^{-}=\left(\Delta^{+}\right)^{\dagger}$ e $\Delta^{--}=\left(\Delta^{++}\right)^{\dagger}$. Estes processos radiativos t em lugar através de loops contendo léptons e os campos escalares $\Delta^{--}$e $\Delta^{-}$, vide figura (4.1). A razões de decaimento são fornecidas na referência [36]:

$$
\operatorname{Br}\left(l_{1} \rightarrow l_{2} \gamma\right)=\frac{25 \alpha\left|\sum_{l} Y_{\Delta l l_{1}}^{\dagger} Y_{\Delta l_{2} l}\right|^{2}}{768 \pi^{3} G_{F}^{2} M_{\Delta}^{4}} \operatorname{Br}\left(l_{1} \rightarrow e \nu_{1} \bar{\nu}_{e}\right)
$$

Usando os limites experimentais

$$
\begin{aligned}
& \operatorname{Br}\left(\mu^{-} \rightarrow e \gamma\right)<2,4 \times 10^{-12} \\
& \operatorname{Br}\left(\tau^{-} \rightarrow \mu \gamma\right)<3,3 \times 10^{-8} \\
& \operatorname{Br}\left(\tau^{-} \rightarrow e \gamma\right)<4,4 \times 10^{-8}
\end{aligned}
$$

os limites para os correspondentes acoplamentos de Yukawa também são fornecidos na tabela (4.1).

O modelo seesaw tipo II abre possibilidades para a observação de novos sinais. Por exemplo, para $Y_{\Delta} \sim \mathcal{O}(1)$, a possível observação de $\mu \rightarrow e \gamma$ pelo experimento MEG [31], impõe que

$$
15 \mathrm{TeV}<M_{\Delta}<50 \mathrm{TeV}
$$

enquanto para $Y_{\Delta} \sim \mathcal{O}\left(10^{-2}\right)$, impõe que $0.15 \mathrm{TeV}<M<0.5 \mathrm{TeV}$. Se $M_{\Delta}$ resulta da ordem de $\mathrm{TeV}$, a não nula carga do campo $\Delta$, abre a possibilidade de observar estes estados nos aceleradores hadrônicos Tevatron e LHC. A produção ou produção associada de partículas $\Delta^{++}$e $\Delta^{+}$com seus possíveis decaimentos deveria levar a sinais facilmente observáveis.

\subsection{Seesaw Tipo III}

Levando em conta o deslocamento induzido sobre $G_{F}$ na equação (3.96), e as modificações nas correntes carregada e neutra equações (3.91)-(3.93) calcularemos os limites atuais sobre a matriz $N N^{\dagger}$.

\subsubsection{Decaimento do $W$}

Nos modelos seesaw tipo III A matriz de mistura não unitária $N$ aparece na

interação da corrente carregada (3.91). Isto modifica a largura de decaimento 
do $W$ em léptons da forma ${ }^{2}$

$$
\Gamma\left(W \rightarrow l_{\alpha} \nu_{\alpha}\right)=\sum_{i} \Gamma\left(W \rightarrow l_{\alpha} \nu_{i}\right)=\frac{G_{F} M_{W}^{3}}{6 \sqrt{2} \pi}\left(N N^{\dagger}\right)_{\alpha \alpha} .
$$

Usando o valor de $G_{F}=G_{F}^{M P} \sqrt{\left(N N^{\dagger}\right)_{e e}\left(N N^{\dagger}\right)_{\mu \mu}}$ dada pela equação (3.96), em (4.18) a seguinte combinação é definida:

$$
f_{\alpha} \equiv \frac{\Gamma\left(W \rightarrow l_{\alpha} \nu_{\alpha}\right) 6 \sqrt{2} \pi}{G_{F}^{M P} M_{W}^{3}}=\frac{\left(N N^{\dagger}\right)_{\alpha \alpha}}{\sqrt{\left(N N^{\dagger}\right)_{e e}\left(N N^{\dagger}\right)_{\mu \mu}}} .
$$

Com os valores experimentais de $\Gamma\left(W \rightarrow e \nu_{e}\right)=(22,41 \pm 0,66) \times 10^{-2} \mathrm{GeV}$, $\Gamma\left(W \rightarrow \mu \nu_{\mu}\right)=(22,03 \pm 0,76) \times 10^{-2} \mathrm{GeV}, \Gamma\left(W \rightarrow \tau \nu_{\tau}\right)=(23,45 \pm 0,89) \times$ $10^{-2} \mathrm{GeV}$, a massa de $W$ e $G_{F}^{M P}=(1,16637 \pm 0,00001) \times 10^{-5}(\mathrm{GeV})^{-2},[22]$, os parâmetros $f_{\alpha}$ tomam os valores:

$$
\begin{aligned}
& f_{e}=1.000 \pm 0.024 \\
& f_{\mu}=0.986 \pm 0.028 \\
& f_{\tau}=1.002 \pm 0.032
\end{aligned}
$$

\subsubsection{Decaimento Invisível do $Z$}

A modificação na corrente neutra fornecida na equação (3.92) leva a

$\Gamma(Z \rightarrow$ invisível $)=\sum_{i j} \Gamma\left(Z \rightarrow \bar{\nu}_{i} \nu_{j}\right)=\frac{G_{F}^{M P} M_{W}^{3}}{6 \sqrt{2} \pi}\left(1+\rho_{t}\right) \sum_{i j}\left|\left(N^{\dagger} N\right)_{i j}^{-1}\right|^{2}$,

onde $\rho_{t} \approx 0.009$ [22] leva em conta correções radiativas principalmente de loops que contém o quark top. Como estas correções dominantes não contém léptons, a dependência da matriz de mistura em (4.21) aparece como um fator global. Usando a seguinte aproximação válida até a primeira ordem em $\epsilon^{\Sigma}$

$$
\sum_{i j}\left|\left(N^{\dagger} N\right)_{i j}^{-1}\right|^{2} \approx 9-2 \sum_{\alpha}\left(N N^{\dagger}\right)_{\alpha \alpha}
$$

\footnotetext{
${ }^{2} \mathrm{~A}$ forma geral de calcular larguras de decaimentos quando existe mistura de neutrinos é dado na referencia [21].
} 
o seguinte vinculo é obtido:

$$
\frac{9-2 \sum_{\alpha}\left(N N^{\dagger}\right)_{\alpha \alpha}}{\sqrt{\left(N N^{\dagger}\right)_{e e}\left(N N^{\dagger}\right)_{\mu \mu}}}=\frac{12 \sqrt{2} \pi \Gamma(Z \rightarrow \text { invisível })}{G_{F}^{M P} M_{W}^{3}\left(1+\rho_{t}\right)}=2.984 \pm 0.009
$$

Como é bem conhecido, este número deve corresponder ao número de neutrinos ativos no LEP. Para obter os valores dos coeficientes $\left(N N^{\dagger}\right)_{\alpha \alpha}$, a expressão em (4.23) pode ser escrita numa forma mais conveniente:

$$
\frac{9 /\left(N N^{\dagger}\right)_{e e}-2 \sum_{\alpha} \frac{\left(N N^{\dagger}\right)_{\alpha \alpha}}{\left(N N^{\dagger}\right)_{e e}}}{\sqrt{\frac{\left(N N^{\dagger}\right)_{\mu \mu}}{\left(N N^{\dagger}\right)_{e e}}}}=2.984 \pm 0.009 .
$$

Agora de (4.19) obtemos as relações

$$
\sqrt{\frac{\left(N N^{\dagger}\right)_{\mu \mu}}{\left(N N^{\dagger}\right)_{e e}}}=f_{\mu} \quad \text { e } \quad \sum_{\alpha} \frac{\left(N N^{\dagger}\right)_{\alpha \alpha}}{\left(N N^{\dagger}\right)_{e e}}=f_{\mu}\left(f_{e}+f_{\mu}+f_{\tau}\right)
$$

Da relação (4.20) em (4.24) é possível determinar o valor de $\left(N N^{\dagger}\right)_{e e}$, que é dado por

$$
\left(N N^{\dagger}\right)_{e e}=1,028 \pm 0,049 .
$$

Usando o valor para $\left(N N^{\dagger}\right)_{e e}$ em (4.25) obtém-se

$$
\begin{aligned}
& \left(N N^{\dagger}\right)_{\mu \mu}=0,999 \pm 0,005 \\
& \left(N N^{\dagger}\right)_{\tau \tau}=1,013 \pm 0,027
\end{aligned}
$$

O que mostra que os elementos diagonais de $N N^{\dagger}$ são muito próximos de 1.

\subsubsection{Testes baseados em universalidade}

Além dos processos considerados até agora, razões de decaimento para léptons, píons e $W$, que são usados para testar a universalidade de interações fracas, podem levar a limites sobre os elementos da matriz $N N^{+}$. O resultado da análise, sempre até ordem $\epsilon^{\Sigma}$, é fornecido na tabela (4.2). Da tabela (4.2), junto com os resultados nas equações (4.26) - (4.27), podemos ver que com $90 \%$ CL, os limites para os coeficientes $\left(N N^{\dagger}\right)_{\alpha \alpha}$ são 


\begin{tabular}{|c|c|c|}
\hline Restrições & Processo & limite \\
\hline$\frac{\left(N N^{\dagger}\right)_{\mu \mu}}{\left(N N^{\dagger}\right)_{e e}}$ & $\frac{\Gamma\left(W \rightarrow \mu \bar{\nu}_{\mu}\right)}{\Gamma\left(W \rightarrow e \bar{\nu}_{e}\right)}$ & $0.997 \pm 0.010$ \\
\hline$\frac{\left(N N^{\dagger}\right)_{\tau \tau}}{\left(N N^{\dagger}\right)_{e e}}$ & $\frac{\Gamma\left(W \rightarrow \tau \bar{\nu}_{\tau}\right)}{\Gamma\left(W \rightarrow e \bar{\nu}_{e}\right)}$ & $1.034 \pm 0.0014$ \\
\hline$\frac{\left(N N^{\dagger}\right)_{\mu \mu}}{\left(N N^{\dagger}\right)_{e e}}$ & $\frac{\Gamma\left(\pi \rightarrow \mu \bar{\nu}_{\mu}\right)}{\Gamma\left(\pi \rightarrow e \nu_{e}\right)}$ & $1.0017 \pm 0.0015$ \\
\hline$\frac{\left(N N^{\dagger}\right)_{\tau \tau}}{\left(N N^{\dagger}\right)_{\mu \mu}}$ & $\frac{\Gamma\left(\tau \rightarrow \pi \bar{\nu}_{\tau}\right)}{\Gamma\left(\pi \rightarrow \mu \bar{\nu}_{\mu}\right)}$ & $0.999 \pm 0.0036$ \\
\hline$\frac{\left(N N^{\dagger}\right)_{\mu \mu}}{\left(N N^{\dagger}\right)_{e e}}$ & $\frac{\Gamma\left(\tau \rightarrow \nu_{\tau} \mu \bar{\nu}_{\mu}\right)}{\Gamma\left(\tau \rightarrow \nu_{\tau} e \bar{\nu}_{e}\right)}$ & $0.999 \pm 0.0020$ \\
\hline$\frac{\left(N N^{\dagger}\right)_{\tau \tau}}{\left(N N^{\dagger}\right)_{\mu \mu}}$ & $\frac{\Gamma\left(\tau \rightarrow \nu_{\tau} e \bar{\nu}_{e}\right)}{\Gamma\left(\mu \rightarrow e \bar{\nu}_{e}\right)}$ & $1.0004 \pm 0.0023$ \\
\hline$\frac{\left(N N^{\dagger}\right)_{\tau \tau}}{\left(N N^{\dagger}\right)_{\mu \mu}}$ & $\frac{\Gamma\left(\tau \rightarrow \nu_{\tau} \mu \bar{\nu}_{\mu}\right)}{\Gamma\left(\mu \rightarrow \nu_{\mu} e \bar{\nu}_{e}\right)}$ & $1.0002 \pm 0.0022$ \\
\hline
\end{tabular}

Tabela 4.2: Restrições sobre $\left(N N^{\dagger}\right)_{\alpha \alpha}$, oriundas do decaimento do bóson $W$ em léptons, decaimento leptônicos e decaimentos de píons.

$$
\begin{aligned}
& \left(N N^{\dagger}\right)_{e e} \simeq 1,001 \pm 0,002 \\
& \left(N N^{\dagger}\right)_{\mu \mu} \simeq 1,002 \pm 0,002, \\
& \left(N N^{\dagger}\right)_{\tau \tau} \simeq 1,002 \pm 0,002
\end{aligned}
$$

onde os limites foram extraídos de [23]. Para os decaimentos leptônicos, a seguinte expressão foi usada $(\alpha \neq \beta)$ :

$$
\Gamma\left(l_{\alpha} \rightarrow \nu_{\alpha} l_{\beta} \bar{\nu}_{\beta}\right)=\frac{\left(G_{F}^{M P}\right)^{2} m_{\alpha}^{5}}{192 \pi^{3}}\left(N N^{\dagger}\right)_{\alpha \alpha}\left(N N^{\dagger}\right)_{\beta \beta},
$$


e para o decaimento dos píons foi usada a expressão:

$$
\Gamma\left(\pi \rightarrow l_{\alpha}+\bar{\nu}_{\alpha}\right)=\frac{\left(G_{F}^{M P}\right)^{2} f_{\pi}^{2} m_{\pi}^{2} m_{\alpha}^{2}}{8 \pi}\left(N N^{\dagger}\right)_{\alpha \alpha}
$$

onde $m_{\pi}$ é a massa do píon e $f_{\pi}$ é o fator de estrutura do píon [22].

\subsubsection{Decaimento do $Z$ em léptons carregados}

Enquanto as análises dos processos nas seções prévias levam a limites sobre os elementos da diagonal de $N N^{\dagger}$, a presença adicional de efeitos que trocam o sabor nos acoplamentos dos férmions carregados, com o bóson $Z$, permitem restringir os elementos fora da diagonal de $N N^{\dagger}$ para processos a nível de árvore. As larguras leptônicas do bóson $Z$ são dadas por

$$
\Gamma\left(Z \rightarrow l_{\alpha} \bar{l}_{\alpha}\right)=\frac{G_{F}^{M P} M_{Z}^{3}}{3 \sqrt{2} \pi}\left(\left|\sin ^{2} \theta_{W}\right|^{2}+\left|-\frac{1}{2}\left(N N^{\dagger}\right)_{\alpha \alpha}^{2}+\sin ^{2} \theta_{W}\right|^{2}\right),
$$

onde utilizamos para obter este resultado

$$
g_{V}^{\alpha \beta}=-\frac{1}{2}\left(N N^{\dagger}\right)_{\alpha \beta}^{2}+2 \sin ^{2} \theta_{W} \delta_{\alpha \beta} \text { e } g_{A}^{\alpha \beta}=-\frac{1}{2}\left(N N^{\dagger}\right)_{\alpha \beta}^{2},
$$

no lugar dos coeficientes usuais do MP tabela (1.1). Para $\alpha \neq \beta$ se obtém

$$
\Gamma\left(Z \rightarrow l_{\alpha} \bar{l}_{\beta}\right)=\frac{G_{F}^{M P} M_{Z}^{3}}{3 \sqrt{2} \pi} \frac{1}{4}\left|\left(N N^{\dagger}\right)_{\alpha \beta}^{2}\right|^{2} .
$$

Agora é possível obter a razões de decaimento até ordem $\epsilon^{\Sigma}$ :

$$
\begin{aligned}
\operatorname{Br}\left(Z \rightarrow l_{\alpha} \bar{l}_{\beta}\right) & =\frac{\Gamma\left(Z \rightarrow l_{\alpha} \bar{l}_{\beta}\right)}{\Gamma\left(Z \rightarrow l_{\lambda} \bar{l}_{\lambda}\right)} \operatorname{Br}\left(Z \rightarrow l_{\lambda} \bar{l}_{\lambda}\right) \\
& =\frac{\left|\left(N N^{\dagger}\right)_{\alpha \beta}\right|^{2}}{2 \sin ^{4} \theta_{W}-\sin ^{2} \theta_{W}+1 / 4} \operatorname{Br}\left(Z \rightarrow l_{\lambda} \bar{l}_{\lambda}\right)
\end{aligned}
$$

onde usamos que $\left(N N^{\dagger}\right)_{\alpha \beta}^{2}=2\left(N N^{\dagger}\right)_{\alpha \beta}$. Apartir de (4.37), as restrições para os elementos fora da diagonal de $N N^{\dagger}$, para processos a nível árvore são fornecidas na tabela (4.3). Nesta tabela, os limites foram obtidos usando os valores experimentais de $\operatorname{Br}\left(Z \rightarrow e^{ \pm} \mu^{\mp}\right)<1,7 \times 10^{-6}, \operatorname{Br}\left(Z \rightarrow e^{ \pm} \tau^{\mp}\right)<$ $9,8 \times 10^{-6}, \operatorname{Br}\left(Z \rightarrow \mu^{ \pm} \tau^{\mp}\right)<1,2 \times 10^{-5}$ e $\operatorname{Br}\left(Z \rightarrow l_{\lambda} \bar{l}_{\lambda}\right)=(3,3658 \pm$ $0,0023) \%,[22]$. 


\begin{tabular}{|c|c|c|}
\hline Restrições & Processo & limite \\
\hline$\left|\left(N N^{\dagger}\right)_{e \mu}\right|$ & $\operatorname{Br}\left(Z \rightarrow e^{ \pm} \mu^{\mp}\right)$ & $<2,5 \times 10^{-3}$ \\
\hline$\left|\left(N N^{\dagger}\right)_{e \tau}\right|$ & $\operatorname{Br}\left(Z \rightarrow e^{ \pm} \tau^{\mp}\right)$ & $<6,1 \times 10^{-3}$ \\
\hline$\left|\left(N N^{\dagger}\right)_{\mu \tau}\right|$ & $\operatorname{Br}\left(Z \rightarrow \mu^{ \pm} \tau^{\mp}\right)$ & $<6,7 \times 10^{-4}$ \\
\hline
\end{tabular}

Tabela 4.3: Vínculos sobre $\left(N N^{\dagger}\right)_{\alpha \beta}$ do decaimento do bóson Z em um par de léptons carregados.

\subsubsection{Decaimento de $\mu \rightarrow 3 e$ e $\tau \rightarrow 3 l$}

A presença de correntes neutras que trocam o sabor no setor de léptons carregados (3.93) fornece transições a nível de árvore do tipo $\mu \rightarrow e^{+} e^{-} e^{-}$ e $\tau \rightarrow 3 l$ as quais ainda não foram observadas experimentalmente. Usando (4.35), para transições do tipo $\mu \rightarrow e^{+} e^{-} e^{-}$é possível obter a razão de decaimento até ordem $\epsilon^{\Sigma}$,

$$
\begin{aligned}
\operatorname{Br}\left(\mu^{-} \rightarrow e^{+} e^{-} e^{-}\right) & =\frac{\Gamma\left(\mu^{-} \rightarrow e^{+} e^{-} e^{-}\right)}{\Gamma\left(\mu^{-} \rightarrow e^{-} \nu_{\mu} \bar{\nu}_{e}\right)} \\
& =\left|\left(N N^{\dagger}\right)_{e \mu}^{2}\right|^{2}\left(3 \sin ^{4} \theta_{W}-2 \sin ^{2} \theta_{W}+1 / 2\right)
\end{aligned}
$$

Analogamente, decaimentos de $\tau$ em $3 e$ e $3 \mu$ são não nulos e dados por:

$$
\begin{aligned}
\operatorname{Br}\left(\tau^{-} \rightarrow l_{\alpha}^{+} l_{\alpha}^{-} l_{\alpha}^{-}\right) & =\frac{\Gamma\left(\tau^{-} \rightarrow l_{\alpha}^{+} l_{\alpha}^{-} l_{\alpha}^{-}\right)}{\Gamma\left(\tau^{-} \rightarrow e^{-} \nu_{\tau} \bar{\nu}_{e}\right)} \operatorname{Br}\left(\tau^{-} \rightarrow e^{-} \nu_{\tau} \bar{\nu}_{e}\right) \\
& =\left|\left(N N^{\dagger}\right)_{\alpha \tau}^{2}\right|^{2}\left(3 \sin ^{4} \theta_{W}-2 \sin ^{2} \theta_{W}+1 / 2\right) \operatorname{Br} \Gamma\left(\tau^{-} \rightarrow e^{-} \nu_{\tau} \bar{\nu}_{e}\right)
\end{aligned}
$$


onde $\alpha=\mu, e$. Por outro lado, decaimentos de $\tau$ em $2 e(\mu)+1 \mu(e)$ são dados por:

$$
\begin{aligned}
\operatorname{Br}\left(\tau^{-} \rightarrow l_{\alpha}^{+} l_{\alpha}^{-} l_{\beta}^{-}\right) & =\frac{\Gamma\left(\tau^{-} \rightarrow l_{\alpha}^{+} l_{\alpha}^{-} l_{\beta}^{-}\right)}{\Gamma\left(\tau^{-} \rightarrow l_{\lambda}^{-} \nu_{\tau} \bar{\nu}_{e}\right)} \operatorname{Br}\left(\tau^{-} \rightarrow l_{\lambda}^{-} \nu_{\tau} \bar{\nu}_{e}\right) \\
& =\left|\left(N N^{\dagger}\right)_{\beta \tau}^{2}\right|^{2}\left(2 \sin ^{4} \theta_{W}-\sin ^{2} \theta_{W}+1 / 4\right) \operatorname{Br}\left(\tau^{-} \rightarrow l_{\lambda}^{-} \nu_{\tau} \bar{\nu}_{e}\right), \\
\operatorname{Br}\left(\tau^{-} \rightarrow l_{\beta}^{+} l_{\alpha}^{-} l_{\alpha}^{-}\right) & =\frac{\Gamma\left(\tau^{-} \rightarrow l_{\beta}^{+} l_{\alpha}^{-} l_{\alpha}^{-}\right)}{\Gamma\left(\tau^{-} \rightarrow e^{-} \nu_{\tau} \bar{\nu}_{e}\right)} \operatorname{Br}\left(\tau^{-} \rightarrow e^{-} \nu_{\tau} \bar{\nu}_{e}\right) \\
& =\frac{1}{2}\left|\left(N N^{\dagger}\right)_{\alpha \tau}^{2}\right|^{2}\left|\left(N N^{\dagger}\right)_{\alpha \beta}^{2}\right|^{2} \operatorname{Br}\left(\tau^{-} \rightarrow e^{-} \nu_{\tau} \bar{\nu}_{e}\right)
\end{aligned}
$$

onde $\alpha, \beta=\mu, e \operatorname{com} \alpha \neq \beta$.

Os limites resultantes destes processos para combinações dos elementos de $N N^{\dagger}$ estão na tabela (4.4). Nesta tabela, pode-se observar que os limites mais fortes para os elementos $\left(N N^{\dagger}\right)_{e \mu},\left(N N^{\dagger}\right)_{e \tau}$ e $\left(N N^{\dagger}\right)_{\mu \tau}$, são produto dos processos $\mu^{-} \rightarrow e^{+} e^{-} e^{-}, \tau^{-} \rightarrow e^{+} e^{-} e^{-}$e $\tau^{-} \rightarrow \mu^{+} \mu^{-} \mu^{-}$, respetivamente. Estes limites são obtidos usando os valores experimentais de $\operatorname{Br}\left(\mu^{-} \rightarrow e^{+} e^{-} e^{-}\right)<10^{-12}, \operatorname{Br}\left(\tau^{-} \rightarrow e^{+} e^{-} e^{-}\right)<2,7 \times 10^{-8}$, $\operatorname{Br}\left(\tau^{-} \rightarrow \mu^{+} \mu^{-} \mu^{-}\right)<2,1 \times 10^{-8}$ e $\operatorname{Br}\left(\tau^{-} \rightarrow e^{-} \nu_{\tau} \bar{\nu}_{e}\right)=17,83 \%$, [22]. Além disso, pode-se observar que o limite mais fraco sobre $\left(N N^{\dagger}\right)_{e \mu}$, é produto do processo $Z \rightarrow e^{ \pm} \mu^{\mp}$, (tabela (4.3)). Enquanto os limites mais fracos sobre $\left(N N^{\dagger}\right)_{e \tau}$ e $\left(N N^{\dagger}\right)_{\mu \tau}$, são produto dos processos $\tau \rightarrow \mu \gamma$ e $\tau \rightarrow e \gamma$, respectivamente. 


\begin{tabular}{|c|c|c|}
\hline Restrições & Processo & limites \\
\hline$\left|\left(N N^{\dagger}\right)_{e \mu}\right|$ & $\mu^{-} \rightarrow e^{+} e^{-} e^{-}$ & $<1,1 \times 10^{-6}$ \\
\hline$\left|\left(N N^{\dagger}\right)_{e \tau}\right|$ & $\tau^{-} \rightarrow e^{+} e^{-} e^{-}$ & $<4,3 \times 10^{-4}$ \\
\hline$\left|\left(N N^{\dagger}\right)_{\mu \tau}\right|$ & $\tau^{-} \rightarrow \mu^{+} \mu^{-} \mu^{-}$ & $<3,8 \times 10^{-4}$ \\
\hline$\left|\left(N N^{\dagger}\right)_{\tau e}\right|$ & $\tau^{-} \rightarrow \mu^{+} \mu^{-} e^{-}$ & $<5,4 \times 10^{-4}$ \\
\hline$\left|\left(N N^{\dagger}\right)_{\tau \mu}\right|\left|\left(N N^{\dagger}\right)_{e \mu}\right|$ & $\tau^{-} \rightarrow e^{+} \mu^{-} \mu^{-}$ & $<1 \times 10^{-4}$ \\
\hline$\left|\left(N N^{\dagger}\right)_{\tau \mu}\right|$ & $\tau^{-} \rightarrow e^{+} e^{-} \mu^{-}$ & $<4,4 \times 10^{-4}$ \\
\hline$\left|\left(N N^{\dagger}\right)_{\tau e}\right|\left|\left(N N^{\dagger}\right)_{\mu e}\right|$ & $\tau^{-} \rightarrow \mu^{+} e^{-} e^{-}$ & $<1 \times 10^{-4}$ \\
\hline$\left|\left(N N^{\dagger}\right)_{e \mu}\right|$ & $\mu^{-} \rightarrow e \gamma$ & $<1,1 \times 10^{-4}$ \\
\hline$\left|\left(N N^{\dagger}\right)_{\mu \tau}\right|$ & $\tau^{-} \rightarrow \mu \gamma$ & $<1,9 \times 10^{-2}$ \\
\hline$\left|\left(N N^{\dagger}\right)_{e \tau}\right|$ & $\tau^{-} \rightarrow e \gamma$ & $<2,4 \times 10^{-2}$ \\
\hline
\end{tabular}

Tabela 4.4: Vínculos sobre $\left(N N^{\dagger}\right)_{\alpha \beta}$ do decaimento de léptons carregados. Nesta tabela pode-se observar que as restrições mais fortes para os coeficientes $\left(N N^{\dagger}\right)_{\alpha \beta}$, são produto dos decaimentos $\mu^{-} \rightarrow e^{+} e^{-} e^{-}, \tau^{-} \rightarrow e^{+} e^{-} e^{-}$, $\tau^{-} \rightarrow \mu^{+} \mu^{-} \mu^{-}$. 


\subsubsection{Lagrangiana completa do seesaw tipo III e $l_{1} \rightarrow$ $l_{2} \gamma$}

Além dos processos considerados ate agora, outro vinculo sobre a matriz $N N^{\dagger}$ pode ser obtido de processos $l_{1} \rightarrow l_{2} \gamma$, mas o operador de $d=6$ indica que estes processos não podem ser obtidos a nível de árvore. Assim, para obter processos $l_{1} \rightarrow l_{2} \gamma$, temos que considerar a lagrangiana completa do modelo seesaw tipo III. A lagrangiana (3.67) expressa em termos das componentes carregadas $\Sigma^{ \pm} \equiv \frac{\Sigma^{1} \mp i \Sigma^{2}}{\sqrt{2}}$ e $\Sigma^{0}=\Sigma^{3}$, é escrita como

$$
\begin{aligned}
\mathcal{L} & =\overline{\Sigma_{R}^{\dagger}} i \not \partial \Sigma_{R}^{\dagger}+\overline{\Sigma_{R}^{-}} i \not \partial \Sigma_{R}^{-}+\overline{\Sigma_{R}^{0}} i \not \partial \Sigma_{R}^{0} \\
& +g\left[W_{u}^{\dagger} \overline{\Sigma_{R}^{0}} \gamma^{u} \Sigma_{R}^{-}-W_{u}^{\dagger} \overline{\Sigma_{R}^{\dagger}} \gamma^{u} \Sigma_{R}^{0}+h . c\right]+g\left[W_{u}^{3} \overline{\Sigma_{R}^{\dagger}} \gamma^{u} \Sigma_{R}^{\dagger}-W_{R}^{3} u \overline{\Sigma_{R}^{-}} \gamma^{u} \Sigma_{R}^{-}\right] \\
& -\frac{1}{2}\left[\overline{\Sigma_{R}^{\dagger}} M_{\Sigma} \Sigma_{R}^{-c}+\overline{\Sigma_{R}^{-}} M_{\Sigma} \Sigma_{R}^{\dagger c}+\overline{\Sigma_{R}^{0}} M_{\Sigma} \Sigma_{R}^{0 c}+h . c\right] \\
& -\left[\overline{\Sigma_{R}^{0}} Y_{\Sigma} \nu_{L}+\sqrt{2} \phi^{0} \overline{\Sigma_{R}^{-}} Y_{\Sigma} l_{L}+\phi^{+} \overline{\Sigma_{R}^{0}} Y_{\Sigma} l_{L}-\sqrt{2} \phi^{+} \overline{\Sigma_{R}^{\dagger}} Y_{\Sigma} \nu_{L}+h . c\right] .
\end{aligned}
$$

Para considerar a mistura dos tripletos com os léptons carregados, é conveniente usar a seguinte definição

$$
\psi \equiv \Sigma_{R}^{c}+\Sigma_{R}^{-},
$$

com o qual a lagrangiana (4.42) é escrita como:

$$
\begin{aligned}
\mathcal{L} & =\bar{\psi} i \not \partial \psi+\overline{\Sigma_{R}^{0}} i \not \partial \Sigma_{R}^{0}-\bar{\psi} M_{\Sigma} \psi-\frac{1}{2}\left[\overline{\Sigma_{R}^{\dagger}} M_{\Sigma} \Sigma_{R}^{-c}+h . c\right] \\
& +g\left[W_{u}^{\dagger} \overline{\Sigma_{R}^{0}} \gamma^{u} P_{R} \psi+W_{u}^{\dagger} \overline{\Sigma_{R}^{0 c}} \gamma^{u} P_{L} \psi+h . c\right]-g W_{u}^{3} \bar{\varphi} \gamma^{u} \psi \\
& -\left[\overline{\bar{\Sigma}_{R}^{0}} Y_{\Sigma} \nu_{L}+\sqrt{2} \phi^{0} \bar{\psi} Y_{\Sigma} l_{L}+\phi^{+} \overline{\Sigma_{R}^{0}} Y_{\Sigma} l_{L}-\sqrt{2} \phi^{+} \overline{\nu_{L}^{c}} Y_{\Sigma}^{T} \psi+h . c\right] .
\end{aligned}
$$

Transições $l_{1} \rightarrow l_{2} \gamma$ são produzidas por processos, em um loop, mediante os bósons $W, Z, h$ e os bósons de Goldstone associados aos bósons $W, Z$, como indica a figura (4.2). Os detalhes do cálculo da matriz de elementos da amplitude de transição é fornecida na referência [25]

$$
\begin{array}{r}
T_{l_{1} \rightarrow l_{2} \gamma}=-i \frac{G_{F}^{S M} e}{\sqrt{2} 16 \pi^{2}} m_{l_{1}} \overline{u_{2}}(p-q)\left(1+\gamma^{5}\right) i \sigma_{\lambda \nu} \epsilon^{\lambda} q^{\nu} u_{1}(p) \\
{\left[c \epsilon_{21}^{\Sigma}+\sum_{i} x_{\nu_{i}}\left(U_{P M N S}\right)_{2 i}\left(U_{P M N S}\right)_{i 1}+\mathcal{O}\left(1 / M_{\Sigma}^{4}\right)\right] .}
\end{array}
$$



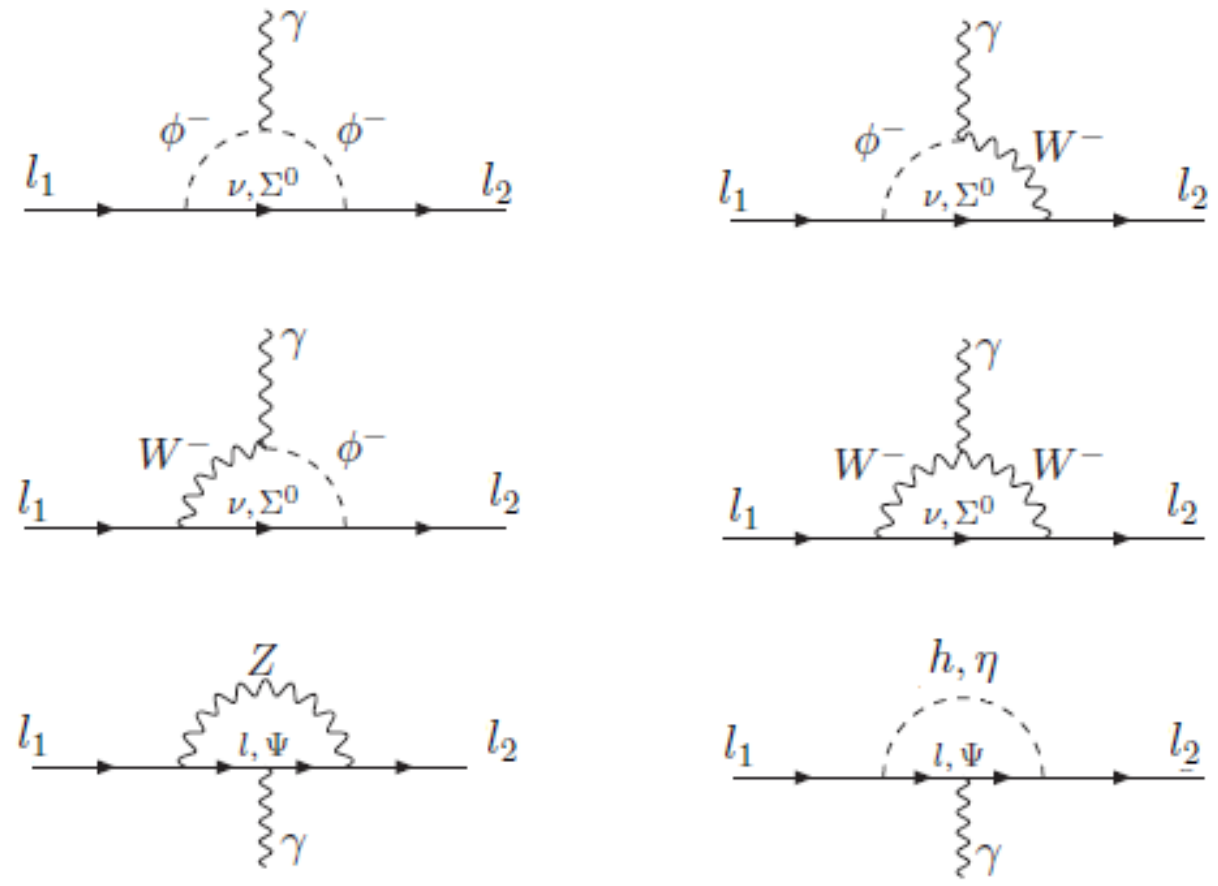

Figura 4.2: Diagramas que contribuem aos processos $l_{1} \rightarrow l_{2} \gamma$. $\phi^{-}$é o bóson de Goldstone associado com o bóson $W^{-}, \eta$ é o bóson de Goldstone associado com o bóson $Z$ e $h$ o bóson de Higgs, [25].

Nesta equação, $c=2,23, x_{\nu_{i}}=\frac{m_{\nu_{i}}^{2}}{M_{W}}$ e $\epsilon_{12}^{\Sigma}$ corresponde aos coeficientes $\epsilon_{e \nu}^{\Sigma}, \epsilon_{e \tau}^{\Sigma}$ e $\epsilon_{\nu \tau}^{\Sigma}$, quando se considera transições $\mu \rightarrow e \gamma, \tau \rightarrow e \gamma$ e $\tau \rightarrow \mu \gamma$, respetivamente. Como se pode observar em (4.45), o segundo termo é a contribuição usual de mistura de férmions [26], enquanto o primeiro termo é a contribuição explícita do tripleto de férmions. Usando a expressão para a amplitude e 
(4.32), a razão entre larguras de decaimento para a transição $l_{1} \rightarrow l_{2} \gamma$ é:

$$
\begin{aligned}
\operatorname{Br}\left(l_{1} \rightarrow l_{2} \gamma\right) & =\frac{\Gamma\left(l_{1} \rightarrow l_{2} \gamma\right)}{\Gamma\left(l_{1} \rightarrow \nu_{1} l_{2} \bar{\nu}_{2}\right)} \operatorname{Br}\left(l_{1} \rightarrow \nu_{1} l_{2} \bar{\nu}_{2}\right) \\
& =\frac{3 \alpha}{32 \pi} \frac{\left|c \epsilon_{21}^{\Sigma}+\sum_{i} x_{\nu_{i}}\left(U_{P M N S}\right)_{2 i}\left(U_{P M N S}\right)_{i 1}\right|^{2}}{\left(N N^{\dagger}\right)_{11}\left(N N^{\dagger}\right)_{22}} \operatorname{Br}\left(l_{1} \rightarrow \nu_{1} l_{2} \bar{\nu}_{2}\right)
\end{aligned}
$$

Agora usando os limites experimentais para as razões das larguras de decaimento [22], as restrições para $N N^{\dagger}$ são fornecidas na tabela (4.4), e são comparadas com as restrições produto de decaimentos puramente leptônicos a nível de árvore.

\subsubsection{Combinação de todos os vínculos}

Das equações (4.29)-(4.31), obtemos os limites para os elementos da diagonal de $N N^{\dagger}$, e dos três primeiros limites fornecidos na tabela (4.4), obtemos os limites sobre os termos fora da diagonal de $N N^{\dagger}$. Assim, obtemos com $90 \%$ de CL que

$$
\left|N N^{\dagger}\right| \approx\left(\begin{array}{ccc}
1,001 \pm 0,002 & <1,1 \times 10^{-6} & <4,3 \times 10^{-4} \\
<1,1 \times 10^{-6} & 1,002 \pm 0,002 & <3,8,2 \times 10^{-4} \\
<4,3 \times 10^{-4} & <3,8 \times 10^{-4} & 1,002 \pm 0,002
\end{array}\right)
$$

Usando a relação obtida em (3.97) entre os elementos da matriz $c^{d=6}$ e da matriz $N N^{\dagger}$, obtemos :

$$
\frac{v^{2}}{2}\left|c^{d=6}\right|_{\alpha \beta}=\left|N N^{\dagger}-1\right|_{\alpha \beta} \approx\left(\begin{array}{ccc}
3 \times 10^{-3} & <1,1 \times 10^{-6} & <4,3 \times 10^{-4} \\
<1,1 \times 10^{-6} & 4 \times 10^{-3} & <3,8 \times 10^{-4} \\
<4,3 \times 10^{-4} & <3,8 \times 10^{-4} & 4 \times 10^{-3}
\end{array}\right)
$$

\subsection{Seesaw Tipo I}

Como acontece no modelo seesaw tipo III, aparece uma matriz $N$ não unitária na definição da corrente neutra, equação (3.29). Disso segue, que se pode impor limites sobre a matriz $N N^{\dagger}$. Para os elementos da diagonal de $N N^{\dagger}$, 
os limites são obtidos da mesma forma que no modelo seesaw tipo III, ou seja, os vínculos originam-se dos dados experimentais de decaimentos dos bósons $Z$ e $W$, testes universais e larguras de decaimento leptônicos . No modelo seesaw tipo III, os limites para os elementos fora da diagonal de $N N^{\dagger}$, são obtidos de processos $\mu^{-} \rightarrow e^{\dagger} e^{-} e^{-}, \tau^{-} \rightarrow e^{\dagger} e^{-} e^{-}$e $\tau^{-} \rightarrow \mu^{\dagger} \mu^{-} \mu^{-}$. Os limites para os elementos fora da diagonal são produto de processos radiativos como $\mu \rightarrow e \gamma, \tau \rightarrow e \gamma$ e $\tau \rightarrow \mu \gamma$. A determinação dos correspondentes limites para $N N^{\dagger}$, são fornecidos na referencia [24]. Com 90\%CL, os limites para $N N^{\dagger}$ são

$$
\left|N N^{\dagger}\right| \simeq\left(\begin{array}{ccc}
0,994 \pm 0.005 & <7,0 \times 10^{-5} & <1,6 \times 10^{-2} \\
<7,0 \times 10^{-5} & 0,995 \pm 0.005 & <1,0 \times 10^{-2} \\
<1,6 \times 10^{-2} & <1,0 \times 10^{-2} & 0,995 \pm 0.005
\end{array}\right)
$$

Usando a relação obtida na equação (3.35) o limite para o coeficiente $c^{d=6}$ é

$$
\frac{v^{2}}{2}\left|c^{d=6}\right|_{\alpha \beta}=\left|N N^{\dagger}-1\right|_{\alpha \beta} \simeq\left(\begin{array}{ccc}
10^{-2} & <7,0 \times 10^{-5} & <1,6 \times 10^{-2} \\
<7,0 \times 10^{-5} & 10^{-2} & <1,0 \times 10^{-2} \\
<1,6 \times 10^{-2} & <1,0 \times 10^{-2} & 10^{-2}
\end{array}\right)
$$




\section{Capítulo 5}

\section{Violação Mínima de Sabor no Modelo Seesaw Tipo III}

\subsection{Introdução}

A possibilidade que a escala da nova física não esteja muito longe da escala eletrofraca abre novas possibilidades para testar a origem da massa dos neutrinos em futuros experimentos. Neste contexto, entretanto, a explicação da massa do neutrino precisa de alguma simetria que assegure os pequenos valores das suas massas comparadas com a massa de outros férmions. Como é bem conhecido, na ausência de novos graus de liberdade, a simetria que assegura esta supressão é a simetria de número total leptônico, que é quebrada pelo operador efetivo de Weinberg (3.74), que gera massa para os neutrinos de Majorana. Porém é concebível que a nova dinâmica que viole sabor leptônico exista em uma escala $\Lambda_{F L}$, que deveria de ser da ordem de $\mathrm{TeV}$, enquanto que o número leptônico total é uma simetria aproximada nesta escala. Genericamente, a baixas energias a lagrangiana de uma teoria completa pode ser expandida como

$$
\mathcal{L}=\mathcal{L}_{M P}+\frac{\alpha^{d=5}}{\Lambda_{L N}} \mathcal{O}^{d=5}+\sum_{i} \frac{\alpha_{i}^{d=6}}{\Lambda_{F L}^{2}} \mathcal{O}_{i}^{d=6}
$$

Nesta expansão, só o operador de dimensão $d=5$ é de Weinberg, responsável para gerar massa aos neutrinos (3.74), e os operadores $\mathcal{O}_{i}^{d=6}$ são de dimensão 
$d=6$ que trocam sabor leptônico, mas conservam NL. As escalas $\Lambda_{F L}, \Lambda_{L N}$ são associadas a nova dinâmica de violação de número leptônico e violação de sabor leptônico, respectivamente. Com $\Lambda_{L N}>>\Lambda_{F L}$ para poder explicar os pequenos valores para as massas dos neutrinos, se a escala $\Lambda_{F L}$ é da ordem de $1 \mathrm{TeV}$. Isto é diferente da implementação descrita nos modelos estudados no capítulo anterior onde $M \sim \Lambda_{L N} \sim \Lambda_{F L}$. A fenomenologia dos operadores de dimensão $d=6$ (no caso de modelos seesaw) associada com a massa do neutrino foi estudada no capítulo anterior. Larguras para processos como $\mu \rightarrow e \gamma$ podem ser em geral consideravelmente grandes se a escala $\Lambda_{F L}$ é da ordem de $\mathrm{TeV}$, mas não é possível predizer a intensidade destes processos de uma forma independente do modelo, desde que a estrutura de sabor dos correspondentes acoplamentos, $\alpha_{i}^{d=6}$, em geral não está relacionada com a massa dos neutrinos, $\alpha_{i}^{d=5}$.

Um tipo de modelo onde a relação pode ser estabelecida é aquele que incorpora violação mínima de sabor (MFV) [27]. MFV assume que a única fonte de violação de sabor em uma teoria efetiva completa é a mesma que no MP: ou seja os acoplamentos de Yukawa, que devem ser incluídos na teoria efetiva como operadores que quebram a simetria de sabor (spúrion de sabor).

Esta hipótese foi primeiramente introduzida no contexto do setor de sabor para os quarks [28]-[30], e isto implica que uma teoria efetiva deve ser construída com campos do MP e acoplamentos de Yukawa dos quarks para cumprir a completa simetria de sabor da $\mathcal{L}_{M P}$, ou seja, os coeficientes dos operadores efetivos de dimensão $d=6$ são combinações específicas dos acoplamentos de Yukawa, que assim determinam a estrutura de sabor. Como resultado, a teoria evita potencialmente grande efeitos de violação de sabor e é muito preditiva para processos que violem sabor.

A mesma hipótese no setor de léptons é mais sutil [27], porque, estritamente falando a única quebra de sabor leptônico na $\mathcal{L}_{M P}$ é feita pelos acoplamentos de Yukawa dos léptons carregados que não induzem efeitos de troca de sabor por si mesmos na ausência de neutrinos massivos. Os adicionais spúrions de sabor necessários para induzir mistura de léptons são dependentes do modelo, desde que eles apareçam nos acoplamentos dos dubletos de léptons com os novos campos nas equações (3.2),(3.37),(3.67). Os autores da referência [27] consideram dois possibilidades no cenário seesaw:

1. Caso mínimo: o spúrion de sabor é o coeficiente do operador de Weinberg, ou seja $\alpha^{d=5}$ na equação (5.1). Como uma consequência, qualita- 
tivamente falando $\alpha^{d=6} \propto \alpha^{d=5^{\dagger}} \alpha^{d=5}$.

2. Caso estendido: Contém neutrinos pesados de mão direita de Majorana (como no modelo seesaw tipo I) e seus acoplamentos com os dubletos leptônicos fornecem os spúrions de sabor, que na ausência de violação CP na matriz de massa dos neutrinos, $\alpha_{i}^{d=6} \propto \alpha_{i}^{d=5}$.

A ideia desenvolvida na referência [27] assume duas condições fundamentais não relacionadas a priori a obedecer:

(a) A hierarquia entre operadores que quebram e preservam número leptônico, ou seja a hierarquia entre as correspondentes escalas, $\Lambda_{F L}<<\Lambda_{L N}$.

(b) A estrutura de sabor dos coeficientes dos operadores $d=6$ fixados pelos coeficientes do operador $d=5$.

Neste capítulo é mostrado um modelo onde se satisfaz as condições (a) e (b) mas a relação entre os operadores $d=5$ e $d=6$ não é nenhuma do tipo considerado na referência [27].

\subsection{MFV Seesaw tipo III}

Dada a lagrangiana da equação (3.67), consideremos apenas dois tripletos de férmions $\Sigma^{w}, \Sigma^{\prime w 1}$ e $M_{\Sigma}, Y_{\Sigma}^{T}$ com a seguinte estrutura de bloco

$$
M_{\Sigma}=\left(\begin{array}{cc}
\mu^{\prime} & \Lambda \\
\Lambda & \mu
\end{array}\right) \quad Y_{\Sigma}^{T} \equiv\left(\begin{array}{cc}
Y^{\dagger} & x Y^{\prime \dagger}
\end{array}\right),
$$

com a equação (5.2) a lagrangiana (3.67) é escrita como:

$$
\begin{aligned}
& \mathcal{L}_{K}=\overrightarrow{\Sigma^{w}}{ }_{R} i \not D \overrightarrow{\Sigma^{w}}{ }_{R}+\overrightarrow{\Sigma^{\prime w}}{ }_{R} i \not D{\overrightarrow{\Sigma^{\prime}}}_{R}, \\
& \mathcal{L}_{Y}=-\overline{L_{L}^{w}} \vec{\tau} \widetilde{\phi} Y^{\dagger} \overrightarrow{\Sigma^{w}}-x \overrightarrow{L_{L}^{w}} \vec{\tau} Y^{\prime \dagger} \widetilde{\phi}{\overrightarrow{\Sigma^{\prime}}}^{w}+h . c, \\
& \left.\mathcal{L}_{\Lambda}=-\frac{\Lambda}{2} \overrightarrow{\left[\overrightarrow{\Sigma^{w} c}\right.} \overrightarrow{\Sigma^{\prime} w}+\overrightarrow{\Sigma^{\prime w c}} \overrightarrow{\Sigma^{w}}\right]-\frac{\mu \overrightarrow{\Sigma^{\prime w c}}}{\Sigma^{\prime w}}-\frac{\mu^{\prime}}{2} \overrightarrow{\Sigma^{w c}} \overrightarrow{\Sigma^{w}},
\end{aligned}
$$

onde os parâmetros insensíveis aos sabor $x, \mu, \mu^{\prime}$ são pequenos comparados com $\Lambda$ e $v$, e $x<<1$, para garantir a simetria aproximada $U(1)_{L N}$, que no limite $\mu, \mu^{\prime}, x \rightarrow 0$ é conservada se $L\left(L^{w}\right)=L\left(\Sigma^{w}\right)=-L\left(\Sigma^{w}\right)=1$.

\footnotetext{
${ }^{1}$ Onde o índice $w$ indica autoestados fracos.
} 


\subsubsection{Hierarquia entre as escalas $\Lambda_{F L}$ e $\Lambda_{L N}$}

Quando os férmions $\Sigma$ e $\Sigma^{\prime}$ são integrados eles fornecem operadores de dimensão $d=5$ e $d=6$, equações (3.74) e (3.78), com coeficientes $c_{\alpha \beta}^{d=5}$ e $c_{\alpha \beta}^{d=6}$. Em termos da equação (5.2) e considerando apenas termos lineares em $x$ os coeficientes são escritos como:

$$
\begin{aligned}
& c_{\alpha \beta}^{d=5}=x\left({\widetilde{Y^{\prime}}}^{T} \frac{1}{\Lambda} Y+Y^{T} \frac{1}{\Lambda} \widetilde{Y}^{\prime}\right)_{\alpha \beta}, \\
& c_{\alpha \beta}^{d=6}=\left(Y^{\dagger} \frac{1}{\Lambda^{\dagger} \Lambda} Y\right)_{\alpha \beta},
\end{aligned}
$$

onde $\widetilde{Y^{\prime}}$ define-se como

$$
\widetilde{Y^{\prime}}=Y^{\prime}-\frac{\mu}{2 x \Lambda} Y
$$

Em geral não existe relação entre os coeficientes $c_{\alpha \beta}^{d=6}$ e $c_{\alpha \beta}^{d=5}$, mas em nosso caso é possível ter uma conexão entre $c_{\alpha \beta}^{d=6}$ e $c_{\alpha \beta}^{d=5}$, para isso podemos reconstruir os vetores de Yukawa $\widetilde{Y^{\prime}}$ e $Y$ de $c_{\alpha \beta}^{d=5}$, e portanto predizer a estrutura de sabor de $c_{\alpha \beta}^{d=6}$.

A quebra de sabor no modelo é produzida por $Y, \widetilde{Y}^{\prime}$ e consequentemente existe violação de sabor inclusive no limite onde o número leptônico é conservado $(x, \mu \rightarrow 0)$ porque $Y$ permanece ativo e assim $c_{\alpha \beta}^{d=6} \neq 0$, equação (5.7).

A estrutura da lagrangiana efetiva na equação (5.1) é obtida, identificando $\Lambda_{F L} \rightarrow \Lambda$ e $\Lambda_{L N} \rightarrow \Lambda / x$ nas equações (5.6) e (5.7). Assim a separação de escalas é obtida pelo pequeno valor de $x$, que restaura a simetria de número leptônico quando $\mu, \mu^{\prime}, x \rightarrow 0$. A escala $\Lambda_{L N}$ não corresponde a nenhuma partícula massiva, enquanto que $\Lambda_{F L}$ corresponde à escala de massa das partículas pesadas $\Sigma$.

\subsubsection{Reconstrução dos acoplamentos de Yukawa $Y$ e $\tilde{Y}^{\prime}$}

Sem perda de generalidade, no que segue, vai-se considerar $\Lambda$ real enquanto que $Y$ e $\tilde{Y}^{\prime}$ são considerados complexos. 
Para reconstruir os acoplamentos $\widetilde{Y^{\prime}}$ e $Y$ de $c^{d=5}$, a seguinte notação é introduzida

$$
Y^{T} \equiv y u_{1},{\widetilde{Y^{\prime}}}^{T} \equiv \widetilde{y}^{\prime} u_{2}
$$

onde $\widetilde{y}^{\prime}$ e $y$ são números reais e $u_{1}$ e $u_{2}$ são vetores tridimensionais complexos de norma um, isto é

$$
<u_{1}, u_{1}>=<u_{2}, u_{2}>=1
$$

O coeficiente $c^{d=5}$ em (5.6) pode ser escrito como

$$
c^{d=5}=\frac{x y \tilde{y}^{\prime}}{\Lambda}\left[u_{1} u_{2}^{T}+u_{2} u_{1}^{T}\right] \equiv \frac{x y \bar{y}^{\prime}}{\Lambda} \widetilde{O} .
$$

De (3.76) a relação entre a matriz de massa para o neutrino e $c^{d=5}$ é

$$
m_{\alpha \beta}=-\frac{v^{2}}{2} c_{\alpha \beta}^{d=5}=-\frac{x y y^{\prime} v^{2}}{2 \Lambda} \widetilde{O}
$$

considerando apenas primeiros termos em $x$ a matriz de massa do neutrino pode ser diagonalizada pela matriz $U=U_{P M N S}$ da seguinte forma:

$$
\frac{x y \widetilde{y^{\prime}} v^{2}}{2 \Lambda} U^{T} \widetilde{O} U=\frac{x y \widetilde{y^{\prime}} v^{2}}{2 \Lambda} \widetilde{O}_{\text {dia }} \equiv-\left(\begin{array}{ccc}
m_{\nu 1} & 0 & 0 \\
0 & m_{\nu 2} & 0 \\
0 & 0 & m_{\nu 3}
\end{array}\right),
$$

onde $\widetilde{O}_{\text {dia }}=U^{T} \widetilde{O} U$ e $m_{\nu i}$ denotam os autovalores de massa do neutrino. Como

$$
U^{\dagger} \widetilde{O}^{\dagger} \widetilde{O} U=\widetilde{O}_{\text {dia }}^{2}
$$

observa-se que $U$ diagonaliza também a matriz $\widetilde{O}^{\dagger} \widetilde{O}$. Assim, a matriz $U_{P M N S}$ é dada pela matriz cujas colunas são os autovetores de $\widetilde{O}^{\dagger} \widetilde{O}$. De (5.13) e (5.14) podemos obter $m_{\nu i}^{2}$ em função dos autovalores de $\widetilde{O}^{\dagger} \widetilde{O}$. Os três autovalores e autovetores de $\widetilde{O}^{\dagger} \widetilde{O}$ são calculados e dados por

$$
\begin{aligned}
\mu_{0} & =0, \quad e_{0}=\frac{u_{1} \times u_{2}}{\sqrt{1-\left|u_{1} \cdot u_{2}\right|^{2}}} \\
\mu_{ \pm} & =(1 \pm \rho)^{2} \quad e_{ \pm}=\frac{1}{\sqrt{2(1 \pm \rho)}}\left[e^{-i \theta / 2} u_{1}^{*} \pm e^{i \theta / 2} u_{2}^{*}\right]
\end{aligned}
$$


onde

$$
<u_{1}, u_{2}>=<u_{2}, u_{1}>^{*}=\rho e^{i \theta} .
$$

Como a matriz $U_{P M N S}$ é dada pela matriz cujas colunas são os autovetores de $\widetilde{O}{ }^{\dagger} \widetilde{O}$, a massa do neutrino e os parâmetros de mistura fixam os autovetores e permitem reconstruir os vetores $u_{1}$ e $u_{2}$ e são dados de (5.16)

$$
\begin{aligned}
& u_{1}^{*}=\frac{e^{i \theta}}{\sqrt{2}}\left[\sqrt{1+\rho} e_{+}+\sqrt{1-\rho} e_{-}\right], \\
& u_{2}^{*}=\frac{e^{-i \theta}}{\sqrt{2}}\left[\sqrt{1+\rho} e_{+}-\sqrt{1-\rho} e_{-}\right],
\end{aligned}
$$

enquanto que $m_{i}$ é fixado por $\rho$ (que depende da hierarquia de massa dos neutrinos). Como podemos absorver $\theta$ na definição de $\Sigma$ e $\Sigma^{\prime}$, podemos escolher $\theta=0$. Mas a reconstrução de $\tilde{Y}^{\prime}$ e $Y$ depende da hierarquia de massa dos neutrinos. Se $m_{\nu_{3}}>m_{\nu_{2}}>m_{\nu_{1}}$, se diz que os neutrinos têm uma hierarquia normal e se $m_{\nu_{3}}<m_{\nu_{1}}<m_{\nu_{2}}$ se diz que os neutrinos têm uma hierarquia inversa.

\subsubsection{Hierarquia Normal}

Neste caso a ordem das massas dos neutrinos é

$$
m_{\nu_{1}}=0, \quad m_{\nu_{2}}=\frac{x y \widetilde{y}^{\prime} v^{2}}{\Lambda}(1-\rho), \quad m_{\nu_{3}}=\frac{x y \widetilde{y}^{\prime} v^{2}}{\Lambda}(1+\rho),
$$

e as colunas de $U$ são dadas como $\left(e_{0}, e_{-}, e_{+}\right)$. Da definição da razão de massas

$$
r=\frac{m_{i j}^{2}}{m_{j k}^{2}},
$$

onde $m_{i j}^{2}=m_{j}^{2}-m_{i}^{2}$ e $m_{i}<m_{j}<m_{k}$, é possível fixar $\rho$ como:

$$
\rho=\frac{\sqrt{1+r}-\sqrt{r}}{\sqrt{1+r}+\sqrt{r}}, \quad r=\frac{m_{12}^{2}}{m_{23}^{2}}=\frac{m_{\nu_{2}}^{2}-m_{\nu_{1}}^{2}}{m_{\nu_{3}}^{2}-m_{\nu_{2}}^{2}}
$$

e os acoplamentos de Yukawa são escritos como:

$$
\begin{aligned}
& Y_{a}=\frac{y}{\sqrt{2}}\left[\sqrt{1+\rho} U_{a 3}^{*}+\sqrt{1-\rho} U_{a 2}^{*}\right], \\
& \widetilde{Y}_{a}^{\prime}=\frac{\widetilde{y}^{\prime}}{\sqrt{2}}\left[\sqrt{1+\rho} U_{a 3}^{*}-\sqrt{1-\rho} U_{a 2}^{*}\right] .
\end{aligned}
$$


Na parametrização padrão para a matriz $U_{P M N S}$ no caso de neutrinos de Majorana,

$$
\begin{aligned}
U_{P M N S} & =\left(\begin{array}{ccc}
c_{12} c_{13} & s_{12} s_{13} & s_{13} e^{-i \delta} \\
-s_{12} c_{23}-c_{12} s_{23} s_{13} e^{i \delta} & c_{12} c_{23}-s_{12} s_{23} s_{13} e^{i \delta} & s_{23} c_{13} \\
s_{12} s_{23}-c_{12} c_{23} s_{13} e^{i \delta} & -c_{12} s_{23}-s_{12} c_{23} s_{13} e^{i \delta} & c_{23} c_{13}
\end{array}\right) \\
& \times\left(\begin{array}{ccc}
e^{-i \alpha} & 0 & 0 \\
0 & e^{i \alpha} & 0 \\
0 & 0 & 1
\end{array}\right),
\end{aligned}
$$

onde a primeira matriz em (5.23) é a parametrização padrão de $U_{P M N S}$ no caso de neutrinos de Dirac, equação (2.108). Existe apenas uma fase de física de Majorana $\alpha$. Isto pode ser explicado olhando o coeficiente $c_{\alpha \beta}^{d=5}$ na equação (5.6). Nesta equação se pode observar que temos 7 fases, três produto do vetor $Y$, três produto do vetor $Y^{\prime}$ e uma da relação $x / \Lambda$, mas das quais não todas são físicas, como pode-se rotar os três dubletos $L_{\alpha}$ e os tripletos $\Sigma$ e $\Sigma^{\prime}$, restam apenas dois fases físicas das quais uma é identificada com a fase CP $\delta$ e a outra com a única fase de Majorana. Usando (5.23) até termos de $\mathcal{O}\left(\sqrt{r}, s_{13}\right)$, obtém-se

$$
Y^{T} \approx y\left(\begin{array}{c}
s_{13} e^{i \delta}+e^{-i \alpha} s_{12} r^{1 / 4} \\
s_{23}\left(1-\frac{\sqrt{r}}{2}\right)+e^{-i \alpha} r^{1 / 4} c_{12} c_{23} \\
c_{23}\left(1-\frac{\sqrt{r}}{2}\right)-e^{-i \alpha} r^{1 / 4} c_{12} s_{23}
\end{array}\right) .
$$

Com os valores dos parâmetros solar $\Delta m_{S U N}^{2} \equiv m_{12}^{2}=0,76 \times 10^{-4} \mathrm{eV}^{2} \mathrm{e}$ atmosférico $\Delta m_{A T M}^{2} \equiv m_{23}^{2}=2,4 \times 10^{-3} \mathrm{eV}^{2}$ [37], se pode fixar a combinação

$$
\left|\frac{x y \widetilde{y}^{\prime} v^{2}}{\Lambda}\right| \sim 0,029 \times \mathrm{eV} \rightarrow\left|\frac{x y \widetilde{y}^{\prime}}{\Lambda}\right| \sim 4.9 \times 10^{-13} \mathrm{TeV}^{-1}
$$

\subsubsection{Hierarquia Inversa}

Neste caso a ordem das massas dos neutrinos é

$$
m_{\nu_{3}}=0, \quad m_{\nu_{1}}=\frac{x y \widetilde{y}^{\prime} v^{2}}{\Lambda}(1-\rho), \quad m_{\nu_{2}}=\frac{x y \widetilde{y}^{\prime} v^{2}}{\Lambda}(1+\rho)
$$

e as colunas de $U$ são dadas por $\left(e_{-}, e_{+}, e_{0}\right)$. Da definição da razão de massas $r$ em (5.20) é possível fixar $\rho$ como:

$$
\rho=\frac{\sqrt{1+r}-1}{\sqrt{1+r}+1}, \quad r=\frac{m_{31}^{2}}{m_{12}^{2}}=\frac{m_{\nu_{1}}^{2}-m_{\nu_{3}}^{2}}{m_{\nu_{2}}^{2}-m_{\nu_{1}}^{2}} .
$$


e os acoplamentos de Yukawa são escritos como:

$$
\begin{aligned}
& Y_{a}=\frac{y}{\sqrt{2}}\left[\sqrt{1+\rho} U_{a 2}^{*}+\sqrt{1-\rho} U_{a 1}^{*}\right] \\
& \tilde{Y}_{a}^{\prime}=\frac{\widetilde{y}^{\prime}}{\sqrt{2}}\left[\sqrt{1+\rho} U_{a 2}^{*}-\sqrt{1-\rho} U_{a 1}^{*}\right] .
\end{aligned}
$$

Usando (5.23) até termos de $\mathcal{O}\left(\sqrt{r}, s_{13}\right)$, obtém-se

$$
Y^{T} \approx \frac{y}{\sqrt{2}}\left(\begin{array}{c}
c_{12} e^{i \alpha}+s_{12} e^{-i \alpha} \\
c_{12}\left(c_{23} e^{-i \alpha}-s_{23} s_{13} e^{i(\alpha-\delta)}\right)-s_{12}\left(c_{23} e^{i \alpha}+s_{23} s_{13} e^{-i(\alpha+\delta)}\right) \\
-c_{12}\left(s_{23} e^{-i \alpha}+c_{23} s_{13} e^{i(\alpha-\delta)}\right)+s_{12}\left(s_{23} e^{i \alpha}-c_{23} s_{13} e^{-i(\alpha+\delta)}\right)
\end{array}\right) .
$$

Com os valores dos parâmetros solar $\Delta m_{\text {solar }}^{2}$ e atmosférico $\Delta m_{\text {atmos }}^{2}$ [37], neste caso se pode fixar a combinação

$$
\left|\frac{x y \widetilde{y}^{\prime} v^{2}}{\Lambda}\right| \sim 0.049 \times \mathrm{eV} \rightarrow\left|\frac{x y \widetilde{y}^{\prime}}{\Lambda}\right| \sim 8.1 \times 10^{-13} \mathrm{TeV}^{-1}
$$

\subsubsection{Escala $\Lambda$ de $\mathcal{O}(\mathrm{TeV})$}

De (4.46) e (5.7) as razões de decaimento podem ser escritas como:

$$
\operatorname{Br}\left(l_{i} \rightarrow l_{j} \gamma\right)=10^{-6}\left(\frac{\mathrm{TeV}}{\Lambda}\right)^{4}\left|u_{1 i}\right|^{2}\left|u_{1 j}\right|^{2} y^{4} \operatorname{Br}\left(l_{i} \rightarrow \nu_{i} l_{j} \bar{\nu}_{j}\right) .
$$

Como $\Lambda_{N L}=\Lambda / x$, consideremos $\Lambda_{N L}$ da escala de grande unificação $\left(10^{12} \times\right.$ $\mathrm{TeV})$, das equações (5.25) e (5.30), obtemos $y \widetilde{y}^{\prime} \sim 0.49$ para a hierarquia normal e $y \widetilde{y}^{\prime} \sim 0.81$ para a hierarquia inversa. Assim, temos a libertade de escolher $y \simeq 1$. Enquanto $\widetilde{y}^{\prime}<1$. Como $u_{1 i}$ é fixo pelos parâmetros de mistura (5.24) e (5.28) com $\left|u_{1 i}\right|^{2}<1$, para $i=\mu, j=e$ e $\left|u_{1(e, \mu)}\right| \approx 10^{-2}$ temos:

$$
\operatorname{Br}(\mu \rightarrow e \gamma) \simeq 10^{-14}\left(\frac{\mathrm{TeV}}{\Lambda}\right)^{4}, \quad \operatorname{Br}(\mu \rightarrow e \gamma)<10^{-12}
$$

Assim, há a possibilidade que a escala $\Lambda$ seja $\mathcal{O}(\mathrm{TeV})$, o que abre a possibilidade de futuras observações de processos $\operatorname{Br}\left(l_{i} \rightarrow l_{j} \gamma\right)$. 


\subsubsection{Autoestados de Massa e suas Interações}

Voltando para a teoria completa, depois que o dubleto de Higgs adquire um VEV não nulo, e usando a definição (4.43), o termo de massa para os léptons se escreve como:

$$
\begin{aligned}
\mathcal{L}_{m} & =-\frac{1}{2}\left(\begin{array}{lll}
{\overline{\nu^{w c}}}_{L} & \bar{N}_{R}^{w} & \bar{N}_{R}^{\prime w}
\end{array}\right) M_{0}\left(\begin{array}{c}
\nu_{L}^{w} \\
N_{R}^{w c} \\
N_{R}^{\prime w c}
\end{array}\right) \\
& -\frac{1}{2}\left(\begin{array}{lll}
\bar{l}_{L} & \bar{\psi}_{L}^{w} & {\overline{\psi^{\prime w}}}_{L}
\end{array}\right) M_{ \pm}\left(\begin{array}{c}
l_{R}^{w} \\
\psi_{R}^{w} \\
\psi_{R}^{\prime w}
\end{array}\right)+\text { h.c }
\end{aligned}
$$

com

$$
M_{0}=\left(\begin{array}{ccc}
0 & \frac{v}{\sqrt{2}} Y^{T} & x \frac{v}{\sqrt{2}} Y^{\prime T} \\
\frac{v}{\sqrt{2}} Y & \mu^{\prime} & \Lambda \\
x \frac{v}{\sqrt{2}} Y^{\prime} & \Lambda & \mu
\end{array}\right), \quad M_{ \pm}=\left(\begin{array}{ccc}
\frac{v}{\sqrt{2}} Y^{l} & v Y^{\dagger} & x v Y^{\prime \dagger} \\
0 & \mu^{\prime} & \Lambda \\
0 & \Lambda & \mu
\end{array}\right) .
$$

Diagonalizando $\mathcal{L}_{m}$ encontra-se três neutrinos de Majorana leves (um deles permanece não massivo) e três léptons carregados com massas fornecidas por

$$
\begin{aligned}
m_{\nu}^{\text {diag }} & =U_{\nu}^{T}\left[-\frac{v^{2}}{2 \Lambda} x\left({\widetilde{Y^{\prime}}}^{T}+Y^{T} \widetilde{Y^{\prime}}\right)\right] U_{\nu}, \\
m_{l}^{\text {diag }} & =\frac{v}{\sqrt{2}}\left(U_{R}^{l}\right)^{\dagger} Y^{l \dagger}\left[1-\frac{V^{2}}{2 \Lambda^{2}} Y^{\dagger} Y\right] U_{L}^{l} .
\end{aligned}
$$

Além disso, são encontrados dois léptons neutros pesados de Majorana e dois léptons carregados pesados com massas $M \approx \Lambda\left(1 \mp \frac{\mu+\mu^{\prime}}{2 \Lambda}\right)$ (obtidos da diagonalização de $M_{\Sigma}$ ).

Usando as matrizes que diagonalizam $\mathcal{L}_{m}$ fornecidas no apêndice $\mathrm{A}$, construímos um estado $N$ com os dois léptons neutros pesados de Majorana e duas combinações de léptons carregados pesados $E_{1}^{-}$e $E_{2}^{+}$que são relacionadas com os autoestados fracos por ${ }^{2}$ :

\footnotetext{
${ }^{2}$ Nas equações 5.37-5.45 são consideradas até a primeira ordem em $x$.
} 


$$
\begin{aligned}
\nu_{L}^{w} & =U^{\nu} \nu+\frac{v}{\sqrt{2} \Lambda} Y^{\dagger} N_{L}+\frac{v}{\sqrt{2} \Lambda}\left[x{\widetilde{Y^{\prime}}}^{\dagger}-\left(\frac{3 \mu+\mu^{\prime}}{4 \Lambda}\right) Y^{\dagger}\right] N_{R}^{c}, \\
l_{L}^{w} & =l_{L}+\frac{v}{\Lambda} Y^{\dagger} E_{1 L}^{-}+\frac{v}{\Lambda}\left[x \widetilde{Y^{\prime}}-\left(\frac{3 \mu+\mu^{\prime}}{4 \Lambda}\right) Y^{\dagger}\right] E_{2 R}^{\dagger c}, \\
l_{R}^{w} & =l_{R}, \\
N_{L}^{w} & =N_{R}^{c}-\left[\frac{\mu-\mu^{\prime}}{4 \Lambda}\right] N_{L}-\frac{v}{\sqrt{2} \Lambda}\left[x \widetilde{Y^{\prime}}-\frac{\mu}{\Lambda} Y\right] U^{\nu} \nu_{L}, \\
N_{L}^{\prime w} & =N_{L}-\left[\frac{\mu-\mu^{\prime}}{4 \Lambda}\right] N_{R}^{c}-\frac{v}{\sqrt{2} \Lambda} Y U^{\nu} \nu_{L}, \\
\psi_{L}^{w} & =E_{2 R}^{\dagger c}-\left[\frac{\mu-\mu^{\prime}}{4 \Lambda}\right] E_{1 L}^{-}-\frac{v}{\Lambda}\left[x \widetilde{Y^{\prime}}-\frac{\mu}{\Lambda} Y\right] l_{L}, \\
\psi_{R}^{w} & =E_{1 R}^{-}-\left[\frac{\mu-\mu^{\prime}}{4 \Lambda}\right] E_{2 L}^{\dagger c}, \\
\psi_{L}^{\prime w} & =E_{1 L}^{-}+\left[\frac{\mu-\mu^{\prime}}{4 \Lambda}\right] E_{2 R}^{\dagger c}-\frac{v}{\Lambda} Y l_{L}, \\
\psi_{R}^{\prime w} & =E_{2 L}^{\dagger c}+\left[\frac{\mu-\mu^{\prime}}{4 \Lambda}\right] E_{1 R}^{-} .
\end{aligned}
$$

As interações carregada e neutra para os estados leves são as mesmas que nas equações (3.91)-(3.93), ou seja, se tem violação da unitariedade e mistura de sabor nas interações carregada e neutra. Mas considerando apenas a primeira ordem no parâmetro $x$ a interação neutra é a mesma que no MP e a interação de corrente carregada é

$$
\mathcal{L}_{W}=\frac{g}{\sqrt{2}}\left[\bar{l}_{L} \gamma^{u} U_{P M N S} \nu_{L}\right] W_{u}^{-}+\text {h.c. }
$$

Além disso as lagrangianas de interação dos tripletos pesados ${ }^{3}$ são escritas

\footnotetext{
${ }^{3}$ As lagrangianas de interação dos tripletos pesados na base de massa são fornecidas no Apêndice, equações (6.15)-(6.17).
} 
como:

$$
\begin{aligned}
\mathcal{L}_{W} & =-g\left[\overline{E_{1}^{-}} \gamma^{u} N W_{u}^{-}-\bar{N} \gamma^{u} E_{2}^{+} W_{u}^{-}\right]+h . c \\
& -\frac{g}{\sqrt{2}}\left[K_{a} \overline{l_{a L}} \gamma^{u} N_{L} W_{u}^{-}+K^{\prime}{ }_{a} \overline{l_{a L}} \gamma^{u} N_{R}^{c} W_{u}^{-}\right]+h . c \\
& +g\left[\widetilde{K_{a}} \overline{\nu_{a L}} \gamma^{u} E_{2 L}^{\dagger} W_{u}^{-}+\widetilde{K^{\prime}}{ }_{a} \overline{\nu_{a L}} \gamma^{u} E_{1 R}^{-c} W_{u}^{-}\right]+h . c \\
\mathcal{L}_{Z} & =g \cos \theta_{w}\left[\overline{E_{1}^{-}} \gamma^{u} E_{1}^{-}-\overline{E_{2}^{\dagger}} \gamma^{u} E_{2}^{\dagger}\right] Z_{u} \\
& +\frac{g}{2 \cos \theta_{w}}\left[\widetilde{K_{a}} \overline{\nu_{a L}} \gamma^{u} N_{L}+\widetilde{K^{\prime}}{ }_{a} \overline{\nu_{a L}} \gamma^{u} N_{R}^{c}\right] Z_{u}+h . c \\
& +\frac{g}{\sqrt{2} \cos \theta_{w}}\left[K_{a} \overline{l_{a L}} \gamma^{u} E_{1 L}^{-}+K^{\prime} \overline{l_{a L}} \gamma^{u} E_{2 R}^{\dagger c}\right] Z_{u}+h . c \\
\mathcal{L}_{\gamma} & =g \cos \theta_{w}\left[\overline{E_{1}^{-}} \gamma^{u} E_{1}^{-}-\overline{E_{2}^{\dagger}} \gamma^{u} E_{2}^{\dagger}\right] A_{u} . \\
\mathcal{L}_{h} & =\frac{g \Lambda}{M_{W}}\left[K_{a} \overline{\nu_{a L}} N_{R}+\widetilde{K^{\prime \prime}}{ }_{a} \overline{\nu_{a L}} N_{L}^{c}\right] h+h . c \\
& +\frac{g \Lambda}{\sqrt{2} M_{W}}\left[K_{a} \overline{l_{a L}} E_{1 R}^{-}+K^{\prime \prime}{ }_{a} \overline{l_{a L}} E_{2 L}^{\dagger c}\right] h+h . c,
\end{aligned}
$$

onde as matrizes $K^{\left({ }^{\prime}\right)\left({ }^{\prime \prime}\right)}$ e $\widetilde{K}^{\left({ }^{\prime}\right)\left({ }^{\prime \prime}\right)}$ são definidas como:

$$
\begin{aligned}
K_{a} & =-\frac{v}{\sqrt{2} \Lambda} Y_{a}^{\dagger}, & \widetilde{K}_{a} & =U_{a c}^{\dagger} K_{c}, \\
k^{\prime}{ }_{a} & =-\frac{v}{\sqrt{2} \Lambda}\left[x Y_{a}^{\prime \dagger}-\left(\frac{3 \mu+\mu^{\prime}}{4 \Lambda}\right) Y_{a}^{\dagger}\right], & {\widetilde{K^{\prime}}}_{a} & =U_{a c}^{\dagger} K_{c}^{\prime}, \\
k^{\prime \prime}{ }_{a} & =-\frac{v}{\sqrt{2} \Lambda}\left[x Y^{\prime \dagger}-\left(\frac{\mu-\mu^{\prime}}{4 \Lambda}\right) Y_{a}^{\dagger}\right], & \widetilde{K}^{\prime \prime}{ }_{a} & =U_{a c}^{\dagger} K^{\prime \prime}{ }_{c} .
\end{aligned}
$$

Como pode ser observado de (5.51) a estrutura de sabor dos acoplamentos dos férmions pesados que conservam número leptônico, $K$ e $\widetilde{K}$, são fixados pelos parâmetros de mistura. Por outro lado, estes acoplamentos são controlados pelo número $v y / \Lambda$, enquanto que $x y \widetilde{y} / \Lambda$ é fixado pela massa do neutrino na equação (5.19) e (5.26). De outro lado os acoplamentos $K^{\left({ }^{\prime}\right)\left({ }^{\prime \prime}\right)}$ e $\widetilde{K}^{\left({ }^{\prime}\right)\left({ }^{\prime \prime}\right)}$ que violam número leptônico dependem de $Y^{\prime}$, que não pode ser fixada pelos parâmetros da mistura. Entretanto é possível observar que estes acoplamentos que violam NL são muito suprimidos devido aos pequenos parâmetros $x, \mu$ e $\mu^{\prime}$. 


\subsection{Fenomenologia}

No que concerne para fenomenologia dos tripletos pesados, violação do número leptônico total aparece em seus decaimentos como uma consequência da presença dos acoplamentos linha e não linha (5.47)-(5.50), assim como da relação de suas massas que dependem de $\mu / \Lambda, \mu^{\prime} / \Lambda$ e da mistura de estados pesados (5.37)-(5.45). Portanto, a pequena violação do número leptônico total implica uma forte hierarquia entre efeitos que conservam e não conservam número leptônico na fenomenologia de colisão de férmions pesados e implica a impossível observação de sinais que violem número leptônico no LHC. Consequentemente, quando os sinais associados com o modelo de estudo são analisados, se tem que concentrar em sinais que conservem número leptônico total.

\section{Produção de Tripletos Pesados}

Como mencionou-se anteriormente, considerando apenas processos que conservem NL, os processos dominantes na produção de tripletos pesados são a produção de pares devido a suas interações de gauge:

$$
\begin{aligned}
& p p \rightarrow \gamma, Z \rightarrow F F^{\prime}, \\
& p p \rightarrow W \rightarrow N F,
\end{aligned}
$$

onde $F, F^{\prime}=E_{1}^{-}, E_{2}^{+}$. Pares neutros $N$ não são produzidos, visto que eles não têm acoplamentos com o bóson $Z$ ou com o fóton, equações (5.48)(5.49). A seções de choque $\sigma$ para os processos em (5.54) não dependem da matriz $K$, isto porque os acoplamentos do tipo $F N W^{-}, F F^{\prime} Z$ ou $F F^{\prime} \gamma$ nas equações (5.47)-(5.50) são independentes da matriz $K$. As seções de choque dos processos em (5.54) são funções bem conhecidas de suas massas [34] como se pode observar na figura (5.1). Nesta figura as seções de choque para $E_{1}^{-} N$ e $E_{2}^{+} N$ têm o mesmo valor, já que os acoplamentos $E_{1}^{-} N W^{-}$e $E_{2}^{+} N W^{-}$em (5.47) têm a mesma forma.

\section{Larguras de Decaimento}

Das equações (5.47)-(5.50) decaimentos dos tripletos pesados podem ser

$$
E_{1}^{-}, E_{2}^{+} \rightarrow W^{-} N
$$




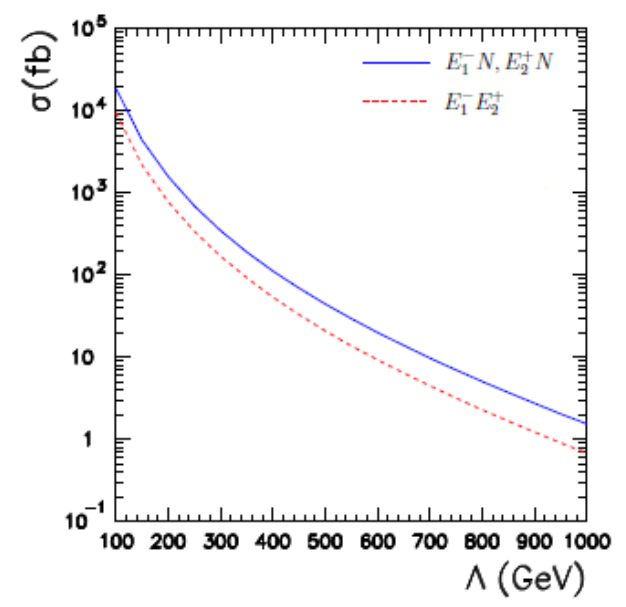

Figura 5.1: Seções de choque para a produção de par de tripletos pesados $E_{1}^{-} N, E_{2}^{+} N$ e $E_{1}^{-} E_{2}^{+}$onde as seções de choque para $E_{1}^{-} N$ e $E_{2}^{+} N$ têm o mesmo valor, [34].

$\mathrm{e}$

$$
F \rightarrow X l_{\alpha}, \quad F \rightarrow X \nu_{\alpha}
$$

onde $F=E_{1}^{-}, E_{2}^{+}, N$ e $X=W, Z, h$. Processos como (5.56), não são permitidos já que os férmions $E_{2}^{+}, E_{1}^{-}$e $N$ têm quase a mesma massa. Para processos como em (5.57), que conservam NL, as larguras para as diferentes 
formas de decaimento são:

$$
\begin{aligned}
\Gamma\left(N \rightarrow l_{a} W^{\dagger}\right) & =\frac{g^{2} \Lambda^{3}}{64 \pi M_{W}^{2}}\left|K_{a}\right|^{2}\left[1-\frac{M_{W}^{2}}{\Lambda^{2}}\right]\left[1+\frac{M_{W}^{2}}{\Lambda^{2}}-2 \frac{M_{W}^{4}}{\Lambda^{4}}\right], \\
\Gamma\left(N \rightarrow \nu_{a} Z\right) & =\frac{g^{2} \Lambda^{3}}{128 \pi \cos ^{2} \theta_{W} M_{Z}^{2}}\left|\widetilde{K}_{a}\right|^{2}\left[1-\frac{M_{Z}^{2}}{\Lambda^{2}}\right]\left[1+\frac{M_{Z}^{2}}{\Lambda^{2}}-2 \frac{M_{Z}^{4}}{\Lambda^{4}}\right], \\
\Gamma\left(N \rightarrow \nu_{a} h\right) & =\frac{g^{2} \Lambda^{3}}{128 \pi M_{Z}^{2}}\left|\widetilde{K}_{a}\right|^{2}\left[1-\frac{M_{h}^{2}}{\Lambda^{2}}\right]^{2}, \\
\Gamma\left(N \rightarrow l_{a} W^{\dagger}\right) & =\frac{g^{2} \Lambda^{3}}{64 \pi M_{W}^{2}}\left|K_{a}\right|^{2}\left[1-\frac{M_{W}^{2}}{\Lambda^{2}}\right]\left[1+\frac{M_{W}^{2}}{\Lambda^{2}}-2 \frac{M_{W}^{4}}{\Lambda^{4}}\right], \\
\Gamma\left(N \rightarrow \nu_{a} Z\right) & =\frac{g^{2} \Lambda^{3}}{128 \pi \cos ^{2} \theta_{W} M_{Z}^{2}}\left|\widetilde{K}_{a}\right|^{2}\left[1-\frac{M_{Z}^{2}}{\Lambda^{2}}\right]\left[1+\frac{M_{Z}^{2}}{\Lambda^{2}}-2 \frac{M_{Z}^{4}}{\Lambda^{4}}\right], \\
\Gamma\left(E_{2}^{\dagger} \rightarrow \nu_{a} W^{\dagger}\right) & =\frac{g^{2} \Lambda^{3}}{32 \pi M_{W}^{2}}\left|\widetilde{K}_{a}\right|^{2}\left[1-\frac{M_{W}^{2}}{\Lambda^{2}}\right]\left[1+\frac{M_{W}^{2}}{\Lambda^{2}}-2 \frac{M_{W}^{4}}{\Lambda^{4}}\right], \\
\Gamma\left(E_{1}^{-} \rightarrow l_{a} Z\right) & =\frac{g^{2} \Lambda^{3}}{64 \pi \cos ^{2} \theta_{W} M_{Z}^{2}}\left|K_{a}\right|^{2}\left[1-\frac{M_{Z}^{2}}{\Lambda^{2}}\right]\left[1+\frac{M_{Z}^{2}}{\Lambda^{2}}-2 \frac{M_{Z}^{4}}{\Lambda^{4}}\right] \\
\Gamma\left(E_{1}^{-} \rightarrow l_{a} h\right) & =\frac{g^{2} \Lambda^{3}}{64 \pi M_{Z}^{2}}\left|K_{a}\right|^{2}\left[1-\frac{M_{h}^{2}}{\Lambda^{2}}\right]^{2} .
\end{aligned}
$$

Usando (5.51) é possível mostrar

$$
\sum_{a=1}^{3}\left|K_{a}\right|^{2}=\sum_{a=1}^{3}\left|\widetilde{K}_{a}\right|^{2}=\frac{y^{2} v^{2}}{2}
$$

e a largura total de decaimento para os férmions $F=N, E_{1}^{-}, E_{2}^{+}$é

$$
\Gamma_{F}^{T O T}=\frac{g^{2} \Lambda y^{2} v^{2}}{64 \pi M_{W}^{2}}\left(1+\mathcal{F}_{F}(\Lambda)\right),
$$

onde $\mathcal{F}_{F}(\Lambda) \rightarrow 0$ para $\Lambda>M_{h}, M_{Z}, M_{W}$. Em um modelo geral seesaw tipo III é possível que a razão das larguras de decaimento de $N$ ou $E_{1}^{-}, E_{2}^{+}$, em léptons carregados de um determinado sabor, sejam nulos. Este não é o caso para o modelo em estudo, equações (5.58)-(5.66); isso por que os acoplamentos de Yukawa fixados pela física do neutrino são não nulas, equações (5.24) 
e (5.29). Portanto, estados finais com dois léptons com sabor definido podem ser produzidos mediante

$$
p p \rightarrow \gamma, Z, W \rightarrow F\left(\rightarrow l_{a} X\right) F^{\prime}\left(\rightarrow l_{b} X^{\prime}\right),
$$

onde $F, F^{\prime}=E_{1}^{-}, N$ e $X, X^{\prime}=W, Z, h$. 


\section{Capítulo 6}

\section{Conclusões}

Enquanto que o único operador de dimensão $d=5$ é comum para os três modelos seesaw estudados, os operadores de dimensão $d=6$ permite diferenciar estes modelos. Estes foram determinamos para os três modelos seesaw. Para os modelos seesaw tipo I e III, os operadores de dimensão $d=6$ efetivos obtidos dão origem a em uma matriz não unitária de mistura, que modifica os acoplamentos dos léptons com os bósons de gauge. Seja $N$ a matriz não unitária que substitui a usual matriz $U_{P M N S}$ na corrente carregada. As correntes léptons- $Z$ e neutrino $-Z$ têm a seguinte estrutura

$J_{Z, L}^{\mu}($ neutrinos $) \propto N^{\dagger} N, \quad J_{Z, L}^{\mu}$ (léptons) $\propto 1 \quad$ para seesaw tipo I, $J_{Z, L}^{\mu}($ neutrinos $) \propto\left(N^{\dagger} N\right)^{-1}, J_{Z, L}^{\mu}$ (léptons $) \propto\left(N N^{\dagger}\right)^{2}$ para seesaw tipo III.

Para o modelo seesaw tipo II, a matriz de mistura permanece unitária, enquanto que os operadores de dimensão $d=6$ geram acoplamentos exóticos de quatro férmions, assim como correções nos parâmetros do potencial do Higgs e correções nos parâmetros de gauge (massa do $Z$ e $W$ ).

Para os três modelos, de forma geral, os coeficientes dos operadores de dimensão $d=6$ são $\left|c^{d=6}\right|=Y^{\dagger} \frac{1}{M^{2}} Y$, onde $Y$ indica os novos acoplamentos de Yukawa e $M$ a escala da nova teoria. Independente do valor de $M$, os limites sobre $Y / M$ são $Y \leq 10^{-1}(M / \mathrm{TeV})$, com maior restrição dependendo do canal especifico, como se pode observar na tabela (4.1) e nas equações (4.48) e (4.50). Para obter tais limites foi necessário considerar os dados experimentais de muitos processos a nível de árvore, assim como processos a um loop $(\mu \rightarrow e \gamma, \tau \rightarrow e \gamma$ e $\tau \rightarrow \mu \gamma)$

No que corresponde ao modelo MFV tipo III, foi possível gerar um desa- 
coplamento entre os coeficientes $c^{d=5}$ e $c^{d=6}$ produto da identificação de duas diferentes escalas $\Lambda_{L N} \sim \Lambda / x$ e $\Lambda_{F V} \sim \Lambda$, onde $\Lambda$ corresponde às massas dos férmions pesados e $x<<1$ para garantir a simetria aproxima $U(1)_{L N}$ que no limite $x \rightarrow 0$ é restaurada. As equações (5.6) e (5.7) indicam que no limite que $x \rightarrow 0$, o coeficiente $c^{d=6} \neq 0$, o qual indica mistura de sabor no setor leptônico carregado. Este coeficiente é determinado pelos parâmetros de mistura e depende da hierarquia de massas normal e invertida, já que neste modelo os acoplamentos de Yukawa $Y$ são reconstruídos em função de tais parâmetros, equações (5.24) e (5.29). A equação (5.32) abre a possibilidade de observações de processos como $l_{2} \rightarrow l_{1} \gamma$ se a escala $\Lambda$ é $\mathcal{O}(\mathrm{TeV})$. Assim no modelo MFV tipo III, o pequeno valor para as massas dos neutrinos é gerado pela larga escala $\Lambda_{L N} \sim \Lambda / x$, sem a necessidade de ter-se partículas massivas com massas da ordem de grande unificação para por ter acoplamentos de Yukawa de $\mathcal{O}(1)$. Os modelos seesaw e em particular o MFV tipo III abrem a possibilidade da observação de fortes sinais que não conservem sabor com acoplamentos de Yukawa de $\mathcal{O}(1)$ e escalas de energia da $\mathcal{O}(\mathrm{TeV})$ em aceleradores sensitivos na escala de TeV tais como o LHC ou ILC.

Da lagrangiana completa no modelo MFV seesaw tipo III (5.3), construímos as interações dos tripletos pesados $N, E_{1}^{-}, E_{2}^{+}$, equações (5.47)(5.50). Apartir destas, considerando apenas processos que conservem $N L$, calculamos as larguras de decaimento de processos como $N \rightarrow l_{a} W^{+}, E_{1}^{-} \rightarrow$ $l_{a} Z$, equações (5.58)-(5.66), estas, são não nulas e portanto, numa colisão $p p$ pode-se obter estados finais com dois léptons de sabor definido, equação 5.69, diferente de o que pode acontecer em um geral modelo seesaw tipo III [35]. 


\section{Apêndice}

\section{Apêndice A: Completa Lagrangiana Seesaw Tipo III na Base de Autoestados de Massa}

Da equação 4.46 a lagrangiana da completa teoria seesaw tipo III pode ser escrita como:

$$
\mathcal{L}=\mathcal{L}_{m}+\mathcal{L}_{C C}+\mathcal{L}_{N C}+\mathcal{L}_{h, \eta}
$$

com

$$
\begin{aligned}
\mathcal{L}_{m} & =-\frac{1}{2}\left(\begin{array}{ll}
\bar{\nu}_{L}^{w c} & \bar{N}_{R}
\end{array}\right) M_{0}\left(\begin{array}{c}
\nu_{L}^{w} \\
N_{R}^{c}
\end{array}\right)-\frac{1}{2}\left(\begin{array}{ll}
\bar{l}_{L}^{w} & \bar{\psi}_{L}
\end{array}\right) M_{ \pm}\left(\begin{array}{c}
l_{R}^{w} \\
\psi_{R}
\end{array}\right)+h . c \\
\mathcal{L}_{C C} & =\frac{g}{\sqrt{2}}\left(\begin{array}{ll}
\bar{l} & \bar{\psi}
\end{array}\right) \gamma^{u} W_{u}^{-}\left[g_{L}^{\prime C C} P_{L}+g_{R}^{\prime C C} P_{R}\right]\left(\begin{array}{c}
\nu \\
N
\end{array}\right) \\
\mathcal{L}_{N C} & =\frac{g}{\cos \theta}\left[\left(\begin{array}{ll}
\bar{l} & \bar{\psi}
\end{array}\right) \gamma^{u} Z_{u}\left[g_{L}^{\prime N C} P_{L}+g_{R}^{\prime N C} P_{R}\right]\left(\begin{array}{c}
l \\
\psi
\end{array}\right)\right. \\
& \left.-e\left(\begin{array}{ll}
\bar{l} & \bar{\psi}
\end{array}\right) \gamma^{u} A_{u}\left(\begin{array}{c}
l \\
\psi
\end{array}\right)\right] \\
\mathcal{L}_{h, \eta} & \left.=\frac{g}{2 M_{W}}\left[\begin{array}{ll}
(\bar{l} & \bar{\psi}
\end{array}\right) h\left[\begin{array}{l}
g_{L}^{\prime h} P_{L}+g_{R}^{\prime h} P_{R}
\end{array}\right]\left(\begin{array}{c}
l \\
\psi
\end{array}\right)\right] \\
& +\frac{g}{2 M_{W}}\left[\left(\begin{array}{ll}
\bar{l} & \bar{\psi}
\end{array}\right) i \eta\left[g_{L}^{\prime \eta} P_{L}+g_{R}^{\prime \eta} P_{R}\right]\left(\begin{array}{c}
l \\
\psi
\end{array}\right)\right]
\end{aligned}
$$


onde

$$
\begin{aligned}
M_{0} & =\left(\begin{array}{cc}
\frac{v}{\sqrt{2}} Y^{l} & v Y^{\dagger} \\
0 & M_{\Sigma}
\end{array}\right), & M_{ \pm}=\left(\begin{array}{cc}
0 & \frac{v}{\sqrt{2}} Y^{T} \\
\frac{v}{\sqrt{2}} Y & M_{\Sigma}
\end{array}\right), \\
g_{L}^{\prime N C} & =\left(\begin{array}{cc}
-\frac{1}{2}+\sin ^{2} \theta & 0 \\
0 & -\cos ^{2} \theta
\end{array}\right), & g_{R}^{\prime N C}=\left(\begin{array}{cc}
\sin ^{2} \theta & 0 \\
0 & -\cos ^{2} \theta
\end{array}\right), \\
g_{L}^{\prime C C} & =\left(\begin{array}{cc}
1 & 0 \\
0 & \sqrt{2}
\end{array}\right), & g_{R}^{\prime C C}=\left(\begin{array}{cc}
0 & 0 \\
0 & \sqrt{2}
\end{array}\right), \\
g_{L}^{\prime h} & =\left(\begin{array}{cc}
m_{l} & 0 \\
v Y_{\Sigma} & 0
\end{array}\right), & g_{R}^{\prime h}=\left(\begin{array}{cc}
m_{l} & v Y_{\Sigma}^{\dagger} \\
0 & 0
\end{array}\right), \\
g_{L}^{\prime \eta} & =\left(\begin{array}{cc}
m_{l} & 0 \\
-v Y_{\Sigma} & 0
\end{array}\right), & g_{R}^{\prime \eta}=\left(\begin{array}{cc}
m_{l} & -v Y_{\Sigma}^{\dagger} \\
0 & 0
\end{array}\right) .
\end{aligned}
$$

Como acontece com as matrizes de massa de Dirac, a matriz de massa de léptons carregados $M_{ \pm}$pode ser diagonalizada por uma transformação biunitária

$$
\left(\begin{array}{c}
l_{L, R} \\
\psi_{L, R}
\end{array}\right)=U_{L, R}\left(\begin{array}{c}
l_{L, R}^{w} \\
\psi_{L, R}^{w}
\end{array}\right)
$$

enquanto que a matriz de massa para léptons neutros $M_{0}$ pode ser diagonalizada por uma simples matriz unitária

$$
\left(\begin{array}{c}
\nu_{L} \\
N_{R}^{c}
\end{array}\right)=U_{0}\left(\begin{array}{c}
\nu_{L}^{w} \\
N_{R}^{c}
\end{array}\right)
$$

Escrevendo a matrizes de mistura como

$$
U_{L} \equiv\left(\begin{array}{cc}
U_{L l l} & U_{L l \psi} \\
U_{L \psi l} & U_{L \psi \psi}
\end{array}\right), \quad U_{R} \equiv\left(\begin{array}{cc}
U_{R l l} & U_{R l \psi} \\
U_{R \psi l} & U_{R \psi \psi}
\end{array}\right), \quad U \equiv\left(\begin{array}{cc}
U_{0 \nu \nu} & U_{0 \nu \Sigma} \\
U_{0 \Sigma \nu} & U_{0 \Sigma \Sigma}
\end{array}\right)
$$

até ordem $\mathcal{O}\left(\left[\left(Y_{\Sigma}, m_{l}\right) / M_{\Sigma}\right]^{2}\right)$ obtém-se:

$$
\begin{aligned}
U_{L l l} & =1-\epsilon^{\Sigma} \\
U_{R l l} & =1 \\
U_{0 \nu \nu} & =\left(1-\epsilon^{\Sigma} / 2\right) U_{P M N S}
\end{aligned}
$$




$$
\begin{aligned}
U_{L l \psi} & =v Y_{\Sigma}^{\dagger} M_{\Sigma}^{-1} V_{\Sigma}, \\
U_{R l \psi} & =v m_{l} Y_{\Sigma}^{\dagger} M_{\Sigma}^{-2} V_{\Sigma}, \\
U_{0 \nu \Sigma} & =\frac{v}{\sqrt{2}} Y_{\Sigma}^{\dagger} M_{\Sigma}^{-1} V_{\Sigma}, \\
U_{L \psi l} & =-v M_{\Sigma}^{-1} Y_{\Sigma}, \\
U_{R \psi l} & =-v M_{\Sigma}^{-2} Y_{\Sigma} m_{l}, \\
U_{0 \Sigma \nu} & =-\frac{v}{\sqrt{2}} M_{\Sigma}^{-1} Y_{\Sigma} U_{0 \nu \nu}, \\
U_{L \psi \psi} & =\left(1-\epsilon^{\prime \Sigma}\right) V_{\Sigma}, \\
U_{R \psi \psi} & =V_{\Sigma}, \\
U_{0 \Sigma \Sigma} & =\left(1-\epsilon^{{ }^{\Sigma}} / 2\right) V_{\Sigma},
\end{aligned}
$$

onde $\epsilon^{\Sigma}=\frac{v^{2}}{2} Y_{\Sigma}^{\dagger} M_{\Sigma}^{-2} Y_{\Sigma}, \epsilon^{\prime \Sigma}=\frac{v^{2}}{2} M_{\Sigma}^{-1} Y_{\Sigma} Y_{\Sigma}^{\dagger} M_{\Sigma}^{-1}$ e $V_{\Sigma}$ é a matriz que diagonaliza a matriz $M_{\Sigma}$. Depois de diagonalizar as matrizes de massa, as equações (6.2)-(6.5) na base auto estados de massa são escritas como:

$$
\begin{aligned}
& \mathcal{L}_{m}=-\frac{1}{2}\left(\begin{array}{ll}
\bar{\nu}_{L}^{w c} & \bar{N}_{R}
\end{array}\right) M_{0}^{\text {diag }}\left(\begin{array}{c}
\nu_{L}^{w} \\
N_{R}^{c}
\end{array}\right)-\frac{1}{2}\left(\begin{array}{ll}
\overline{l w}_{L} & \bar{\varphi}_{L}
\end{array}\right) M_{ \pm}^{\text {diag }}\left(\begin{array}{c}
l_{R}^{w} \\
\psi_{R}
\end{array}\right)+h . c, \\
& \mathcal{L}_{C C}=\frac{g}{\sqrt{2}}\left(\begin{array}{ll}
\bar{l} & \bar{\psi}
\end{array}\right) \gamma^{u} W_{u}^{-}\left[g_{L}^{C C} P_{L}+g_{R}^{C C} P_{R}\right]\left(\begin{array}{c}
\nu \\
N
\end{array}\right) \\
& \mathcal{L}_{N C}=\frac{g}{\cos \theta}\left[\left(\begin{array}{ll}
\bar{l} & \bar{\psi}
\end{array}\right) \gamma^{u} Z_{u}\left[g_{L}^{N C} P_{L}+g_{R}^{N C} P_{R}\right]\left(\begin{array}{c}
l \\
\psi
\end{array}\right)\right. \\
& \left.-e\left(\begin{array}{ll}
\bar{l} & \bar{\psi}
\end{array}\right) \gamma^{u} A_{u}\left(\begin{array}{c}
l \\
\psi
\end{array}\right)\right] \\
& \mathcal{L}_{h, \eta}=\frac{g}{2 M_{W}}\left[\left(\begin{array}{ll}
\bar{l} & \bar{\psi}
\end{array}\right) h\left[g_{L}^{h} P_{L}+g_{R}^{h} P_{R}\right]\left(\begin{array}{c}
l \\
\psi
\end{array}\right)\right] \\
& +\frac{g}{2 M_{W}}\left[\left(\begin{array}{ll}
\bar{l} & \bar{\psi}
\end{array}\right) i \eta\left[g_{L}^{\eta} P_{L}+g_{R}^{\eta} P_{R}\right]\left(\begin{array}{c}
l \\
\psi
\end{array}\right)\right],
\end{aligned}
$$


com

$$
\begin{aligned}
& g_{L}^{N C}=U_{L}^{\dagger} g_{L}^{\prime N C} U_{L}=\left(\begin{array}{cc}
-\frac{1}{2}+\sin ^{2} \theta-\epsilon_{\Sigma} & \frac{v}{2} Y_{\Sigma}^{\dagger} M_{\Sigma}^{-1} V_{\Sigma} \\
V_{\Sigma}^{\dagger}\left[\frac{v}{2} M_{\Sigma}^{-1} Y_{\Sigma}\right] & V_{\Sigma}^{\dagger}\left[-\cos ^{2} \theta\right] V_{\Sigma}
\end{array}\right), \\
& g_{R}^{N C}=U_{R}^{\dagger} g_{R}^{\prime N C} U_{R}=\left(\begin{array}{cc}
\sin ^{2} \theta & v m_{l} Y_{\Sigma}^{\dagger} M_{\Sigma}^{-2} V_{\Sigma} \\
v V_{\Sigma}^{\dagger} M_{\Sigma}^{-2} Y_{\Sigma} m_{l} & -\cos ^{2} \theta
\end{array}\right) \text {, } \\
& g_{L}^{C C}=U_{L}^{\dagger} g_{L}^{\prime C C} U_{0}=\left(\begin{array}{cc}
\left(1+\epsilon_{\Sigma}\right) U_{0 \nu \nu} & -\frac{v}{\sqrt{2}} Y_{\Sigma}^{\dagger} M_{\Sigma}^{-1} V_{\Sigma} \\
0 & \sqrt{2} V_{\Sigma}^{\dagger}\left(1-\frac{\epsilon^{\prime}}{2}\right) V_{\Sigma}
\end{array}\right), \\
& g_{R}^{C C}=U_{R}^{\dagger} g_{R}^{\prime C C} U_{0}=\left(\begin{array}{cc}
0 & -m_{l}^{\dagger} Y_{\Sigma}^{\dagger} M_{\Sigma}^{-2} V_{\Sigma} \\
-\frac{v}{\sqrt{2}} V_{\Sigma}^{\dagger} M_{\Sigma}^{-1} Y_{\Sigma} U_{0 \nu \nu} & V_{\Sigma}^{\dagger}\left(1-\frac{\epsilon^{\prime}}{2}\right) V_{\Sigma}
\end{array}\right), \\
& g_{L}^{h}=U_{R}^{\dagger} g_{L}^{\prime h} U_{L}=\left(\begin{array}{cc}
m_{l}\left(1-3 \epsilon_{\Sigma}\right) & v m_{l} Y_{\Sigma}^{\dagger} M_{\Sigma}^{-1} V_{\Sigma} \\
v V_{\Sigma}^{\dagger} Y_{\Sigma}\left(1-\epsilon_{\Sigma}\right)+v V_{\Sigma}^{\dagger} M_{\Sigma}^{-2} Y_{\Sigma}^{\dagger} m_{l}^{2} & 2 V_{\Sigma}^{\dagger} M_{\Sigma} \epsilon^{\prime}
\end{array}\right), \\
& g_{R}^{h}=U_{L}^{\dagger} g_{R}^{\prime h} U_{R}=\left(\begin{array}{cc}
\left(1-3 \epsilon_{\Sigma}\right) m_{l} & \left(1-\epsilon_{\Sigma}\right)\left[v m_{l}^{2} Y_{\Sigma}^{\dagger} M_{\Sigma}^{-2} V_{\Sigma}+v Y_{\Sigma}^{\dagger} V_{\Sigma}\right] \\
v M_{\Sigma}^{-1} Y_{\Sigma} m_{l} & v^{2} M_{\Sigma}^{-1} Y_{\Sigma} Y_{\Sigma}^{\dagger} V_{\Sigma}
\end{array}\right) \\
& g_{L}^{\eta}=U_{L}^{\dagger} g_{L}^{\prime \eta} U_{0}=\left(\begin{array}{cc}
m_{l}\left(1+\epsilon_{\Sigma}\right) & v m_{l} Y_{\Sigma}^{\dagger} M_{\Sigma}^{-1} V_{\Sigma} \\
-v V_{\Sigma}^{\dagger} Y_{\Sigma}\left(1-\epsilon_{\Sigma}\right)+v V_{\Sigma}^{\dagger} M_{\Sigma}^{-2} Y_{\Sigma}^{\dagger} m_{l}^{2} & -2 V_{\Sigma}^{\dagger} M_{\Sigma} \epsilon^{\prime}
\end{array}\right), \\
& g_{R}^{\eta}=U_{R}^{\dagger} g_{R}^{\prime \eta} U_{0}=\left(\begin{array}{cc}
\left(1+\epsilon_{\Sigma}\right) m_{l} & \left(1-\epsilon_{\Sigma}\right)\left[v m_{l}^{2} Y_{\Sigma}^{\dagger} M_{\Sigma}^{-2} V_{\Sigma}+v Y_{\Sigma}^{\dagger} V_{\Sigma}\right] \\
v M_{\Sigma}^{-1} Y_{\Sigma} m_{l} & v^{2} M_{\Sigma}^{-1} Y_{\Sigma} Y_{\Sigma}^{\dagger} V_{\Sigma}
\end{array}\right)
\end{aligned}
$$




\section{Referências Bibliográficas}

[1] S. L. Glashow, Partial-Symmetries of weak interactions, Nucl. Phys. 22, 579-588 (1961).

[2] A. Salam, Weak and electromagnetic interaction, In N. Svartholm, Elementary Particle Theory, Almqvist and Wiksell, 1968.

[3] S. Weinberg, A model of leptons, Phys. Rev. Lett. 19, 1264-1266 (1967).

[4] F. J. Hasert, H. Faissner, W. Krenz et al., Phys. Lett. B 46, 121-124 (1973); F. J. Hasert et al. [ Gargamelle Neutrino Collaboration ], Phys. Lett. B 46, 138-140 (1973).

[5] G. Arnison et al. [ UA1 Collaboration ], Phys. Lett. B 122, 103-116 (1983).

[6] G. Arnison et al. [ UA1 Collaboration ], Phys. Lett. B 126, 398-410 (1983).

[7] Y. Fukuda et al. [Super-Kamiokande Collaboration], Phys. Rev. Lett. 81, 1562 (1998); Q. R. Ahmad et al. [SNO Collaboration], Phys. Rev. Lett. 89, 011301 (2002).

[8] S. Weinberg, Baryon and lepton nonconserving processes, Phys. Rev. Lett. 43, 1566 (1979).

[9] C. Giunti and C. W. Kim, Fundamentals of Neutrino Physics and Astrophysics, Oxoford University Press, 2007.

[10] G. 't Hooft, Renormalizable Lagrangians for massive Yang-Mills fields, Nucl.Phys., B 35, 167-188 (1971). 
[11] P. W. Higgs, Spontaneous symmetry breakdown without massless bosons, Phys. Rev. 145, 1156-1163 (1966).

[12] M. C. Gonzalez-Garcia and M. Maltoni, Phenomenology with Massive Neutrinos, Phys. Rept. 460, 1-129 (2008).

[13] S. L. Adler, Anomalies to All Orders, 't Hooft, G. (ed.): 50 years of Yang-Mills theory,(2004), arXiv:hep-th/0405040v6.

[14] B. Pontecorvo, inverse beta process, Chalk River Lab. PD-205 report (1946).

[15] B. Pontecorvo, inverse beta process, Camb. Monogr. Part. Phys. Nucl. Phys. Cosmol. 1, 1-25 (1991).

[16] B. Pontecorvo, Mesonium and anti-mesonium, Sov. Phys. JETP 6, 429 (1957).

[17] B. Pontecorvo, Inverse beta processes and nonconservation of lepton charge, Sov. Phys. JETP 7, 172 (1958).

[18] M. Gell-Mann, P. Ramond and R. Slansky, Proceedings of the Supergravity Stony Brook Workshop, Eds. P.van Niewenhuizen and D.Freedman, 1979.

[19] M. E. Peskin and D. V. Schroeder, An Introdution to Quantum Field Theory, Harper Collins Publishers, 1995.

[20] T. P. Cheng and L. F. Li, Gauge theory of Elementary Particle Physics, Clarendon Press, 1984.

[21] C. Giunti, Flavor Neutrinos States, [arXiv:hep-ph/0402217v1], (2004).

[22] J. Beringer et al. (Particle Data Group), Phys. Rev. D 86, 010001 (2012).

[23] A. Pich, The Standard Model of Electroweak Interactions, IFIC/0513, FTUV/05-0201, [arXiv: hep-ph/0502010v1], (2005).

[24] S. Antusch, C. Biggio, E. Fernandez-Martinez, M. B. Gavela and J. Lopez-Pavon, Unitarity of the Leptonic Mixing Matrix, JHEP 0610, 084 (2006). 
[25] A. Abada, C. Biggio, F. Bonnet, M.B. Gavela, T. Hambye, $\mu \rightarrow e \gamma$ and $\tau \rightarrow l \gamma$ decays in the fermion triplet seesaw model, Phys. Rev. D 78, 033007 (2008).

[26] S. M. Bilenky and S. T. Petcov, Massive neutrinos and neutrino oscillations, Rev. Mod. Phys. 59, 671 (1987) [Erratum-ibid. 61, 169 (1989)].

[27] V. Cirigliano, B. Grinstein, G. Isidori and M. B. Wise, Minimal flavor violation in the lepton sector, Nucl. Phys. B 728, 121 (2005).

[28] R. S. Chivukula and H. Georgi, Composite-technicolor standard model, Phys. Lett. B 188, 99 (1987).

[29] A. J. Buras, P. Gambino, M. Gorbahn, S. Jager and L. Silvestrini, Universal Unitarity Triangle and Physics Beyond the Standard Model, Phys. Lett. B 500, 161 (2001).

[30] G. D'Ambrosio, G. F. Giudice, G. Isidori and A. Strumia, Minimal Flavour Violation: an effective field theory approach, Nucl. Phys. B 645, 155 (2002).

[31] S. Ritt et al [MEG Collaboration], Nucl. Phys. Proc. Suppl. 162, 279 (2006).

[32] A. De Rujula, M.B. Gavela, P. Hernandez and E. Masso, The selfcouplings of vector bosons: does LEP-1 obviate LEP-2?, Nucl. Phys. B 384, 3 (1992).

[33] E. Ma, U. Sarkar, Neutrino Masses and Leptogenesis with Heavy Higgs Triplets, Phys.Rev.Lett. 80, 5716-5719 (1998).

[34] F. del Aguila, J. A. Aguilar-Saavedra, Distinguishing seesaw models at LHC with multi-lepton signals, Nucl. Phys. B 813, 22-90 (2009).

[35] F. del aguila, J.A.Aguilar-Saavedra, Electroweak scale seesaw and heavy Dirac neutrino signals at LHC, Phys. Lett. B 672, 158-165 (2009).

[36] J. Bernabeu, A. Pich and A. Santamaria, $\mu^{-} \rightarrow e^{-} \gamma$ decay in the scalar triplet model, Phys. Lett. B 148, 229-233 (1984). 
[37] Andrea Romanino, Neutrino Physics, CERN Yellow Report CERN001, 153-182 (2012).

[38] H. M. Georgi, S. L. Glashow and S. Nussinov, Unconventional model of neutrino masses, Nucl. Phys. B 193, 279-316 (1981).

[39] R. N. Mohapatra, Rare decays of the $\tau$ lepton as a probe of the leftright-symmetric theories of weak interactions, Phys. Rev. D 46, 29902995 (1992).

[40] J. F. Gunion, J. Grifols, A. Mendez, B. Kayser, F.Olness, Higgs bosons in left-right-symmetric models, Phys. Rev. D 40, 1546-1561 (1989).

[41] F. Reines and C.L. Cowan, Detection of the Free Neutrino, Phys. Rev. 92, 830-831 (1953).

[42] C.L. Cowan et al., Science 124, 103 (1956).

[43] M. Koshiba, Observational neutrino astrophysics, Phys. Rep, 220, 229381 (1992).

[44] J. Boger et al. [ SNO Collaboration], Nucl. Instrum. Meth., A 449, 172207 (2000).

[45] Y. Fukuda et al., Measurements of the Solar Neutrino Flux from Super-Kamiokande's First 300 Days, Phys. Rev. Lett. 81, 1158-1162 (1998).

[46] R. Davis, Solar Neutrinos. II. Experimental, Phys. Rev. Lett. 12, 303-305 (1964). 